\title{
Rapid and stable mobilization of fully functional spike-specific CD8+ T cells preceding a mature humoral response after SARS-CoV-2 mRNA vaccination
}

\section{Robert Thimme ( $\nabla$ robert.thimme@uniklinik-freiburg.de )}

Freiburg Freiburg University Medical Center, Faculty of Medicine, University of Freiburg https://orcid.org/0000-0003-1417-4135

\section{Valerie Oberhardt}

Department of Medicine II, Freiburg Freiburg University Medical Center, Faculty of Medicine, University of Freiburg, Freiburg, Germany

\section{Hendrik Luxenburger}

Department of Medicine II (Gastroenterology, Hepatology, Endocrinology and Infectious Diseases),

Freiburg University Medical Center, Faculty of Medicine, University of Freiburg, Freiburg, Germany https://orcid.org/0000-0001-7182-8620

\section{Janine Kemming}

Department of Medicine II, Freiburg Freiburg University Medical Center, Faculty of Medicine, University of Freiburg, Freiburg, Germany

\section{Isabel Schulien}

Department of Medicine II, Freiburg Freiburg University Medical Center, Faculty of Medicine, University of Freiburg, Freiburg, Germany

\section{Kevin Ciminski}

University Medical Center Freiburg https://orcid.org/0000-0001-5397-7497

\section{Sebastian Giese}

University Medical Center Freiburg

\section{Iga Janowska}

Department of Rheumatology and Clinical Immunology

\section{Julian Staniek}

Department of Rheumatology and Clinical Immunology

\section{Katharina Wild}

Department of Medicine II, Freiburg Freiburg University Medical Center, Faculty of Medicine, University of Freiburg, Freiburg, Germany

Julia Lang-Meli

Department of Medicine II

\section{Benedikt Csernalabics}


Department of Medicine II

\section{Kristi Basho}

Department of Medicine II

\section{Mircea Marinescu}

Department of Medicine II

\section{Jonas Fuchs}

Department of Medicine II

\section{Fernando Topfstedt}

Department of Rheumatology and Clinical

\section{Ales Janda \\ Department of Pediatrics and Adolescent Medicine \\ Oezlem Sogukpinar}

Department of Medicine II (Gastroenterology, Hepatology, Endocrinology and Infectious Diseases), Freiburg University Medical Center, Faculty of Medicine, University of Freiburg, Freiburg, Germany

\section{Hanna Hilger}

Department of Medicine II

\section{Katarina Stete}

Department of Medicine II

\section{Florian Emmerich}

Institute for Transfusion Medicine and Gene Therapy, Freiburg University Medical Center, Faculty of Medicine, University of Freiburg, Germany

\section{Bertram Bengsch}

University Medical Center Freiburg https://orcid.org/0000-0003-2552-740X

\section{Sagar Sagar}

Department of Medicine II

\section{Cornelius Waller}

University of Freiburg https://orcid.org/0000-0002-5777-0212

\section{Siegbert Rieg}

Department of Medicine II (Gastroenterology, Hepatology, Endocrinology and Infectious Diseases), Freiburg University Medical Center, Faculty of Medicine, University of Freiburg, Freiburg, Germany

\section{Katharina Zoldan}

Department of Medicine II

\section{Tobias Boettler}

Department of Medicine II, Medical Center - University of Freiburg, Germany https://orcid.org/00000002-1195-055X

\section{Georg Kochs}

Institute of Virology, Medical Centre, Faculty of Medicine, University of Freiburg, Freiburg, Germany https://orcid.org/0000-0003-0187-559X

\section{Martin Schwemmle}


University Medical Center Freiburg https://orcid.org/0000-0002-2972-6855

\section{Marta Rizzi}

University Medical Center Freiburg

\section{Christoph Neumann-Haefelin}

Department of Medicine II (Gastroenterology, Hepatology, Endocrinology and Infectious Diseases), Freiburg University Medical Center, Faculty of Medicine, University of Freiburg, Freiburg, Germany https://orcid.org/0000-0001-7351-1387

\section{Maike Hofmann}

University Hospital Freiburg https://orcid.org/0000-0001-8410-8833

\section{Biological Sciences - Article}

Keywords: SARS-CoV-2, COVID-19, CD8+ T-cell response, mRNA vaccination, bnt162b2, Comirnaty, antiviral immunity, immunological memory, adaptive immune response, CD4+ $T$ cell response, $B$ cell response

Posted Date: May 11th, 2021

DOl: https://doi.org/10.21203/rs.3.rs-505193/v1

License: (c) (i) This work is licensed under a Creative Commons Attribution 4.0 International License. Read Full License

Version of Record: A version of this preprint was published at Nature on July 28th, 2021. See the published version at https://doi.org/10.1038/s41586-021-03841-4. 
1 Rapid and stable mobilization of fully functional spike-specific CD8+ T cells 2 preceding a mature humoral response after SARS-CoV-2 mRNA vaccination

4 Valerie Oberhardt ${ }^{1,2 *}$, Hendrik Luxenburger ${ }^{1,3 *}$ Janine Kemming $^{1,2 *}$, Isabel Schulien ${ }^{1 *}$, 5 Kevin Ciminski ${ }^{4}$, Sebastian Giese ${ }^{4}$, Iga Janowska ${ }^{5}$, Julian Staniek ${ }^{5}$, Katharina Wild ${ }^{1,6}$, 6 Julia Lang-Meli1 ${ }^{13}$, Benedikt Csernalabics ${ }^{1}$, Kristi Basho ${ }^{1}$, Mircea Stefan Marinescu ${ }^{1}$, 7 Jonas Fuchs $^{4}$, Fernando Topfstedt ${ }^{5}$, Ales Janda ${ }^{7}$, Oezlem Sogukpinar ${ }^{1}$, Hanna Hilger ${ }^{1}$, 8 Katarina Stete ${ }^{1}$, Florian Emmerich ${ }^{8}$, Bertram Bengsch ${ }^{1,9}$, Cornelius F. Waller ${ }^{10}$, 9 Siegbert Rieg ${ }^{1}$, Sagar ${ }^{1}$, Katharina Zoldan ${ }^{1}$, Tobias Boettler ${ }^{1,11}$, Georg Kochs ${ }^{4}$, Martin Schwemmle ${ }^{4}$, Marta Rizzi ${ }^{5}$, Robert Thimme ${ }^{1 \# \S}$, Christoph Neumann-Haefelin ${ }^{1 \# \S}$, Maike 11 Hofmann ${ }^{1 \# \S}$

13 'Department of Medicine II (Gastroenterology, Hepatology, Endocrinology and 14 Infectious Diseases), Freiburg University Medical Center, Faculty of Medicine, 15 University of Freiburg, Freiburg, Germany

16 2Faculty of Biology, University of Freiburg, Freiburg, Germany

17 IMM-PACT, Faculty of Medicine, University of Freiburg, Freiburg, Germany

$18{ }^{4}$ Institute of Virology, Freiburg University Medical Center, Faculty of Medicine, 19 University of Freiburg, Freiburg, Germany

$20{ }^{5}$ Department of Rheumatology and Clinical Immunology, Freiburg University Medical 21 Center, Faculty of Medicine, University of Freiburg, Freiburg, Germany

$22{ }^{6}$ Faculty of Chemistry and Pharmacy, University of Freiburg, Freiburg, Germany

23 'Department of Pediatrics and Adolescent Medicine, Ulm University Medical Center, 24 Ulm, Germany

$25{ }^{8}$ Institute for Transfusion Medicine and Gene Therapy, Freiburg University Medical 26 Center, Faculty of Medicine, University of Freiburg, Germany 
$27{ }^{9}$ Signalling Research Centres BIOSS and CIBSS, University of Freiburg, Freiburg,

28 Germany

$29{ }^{10}$ Department of Haematology, Oncology \& Stem Cell Transplantation, Freiburg

30 University Medical Center, Faculty of Medicine, University of Freiburg

$31{ }^{11}$ Berta-Ottenstein Programme, Faculty of Medicine, University of Freiburg

32

33 *equally contributing first authors; \#equally contributing last authors; §corresponding 34 authors

35

36 Materials and Correspondence:

37 Maike Hofmann: maike.hofmann@uniklinik-freiburg.de

38 Christoph Neumann-Haefelin: christoph.neumann-haefelin@uniklinik-freiburg.de

39 Robert Thimme: robert.thimme@uniklinik-freiburg.de

40 Hugstetter Straße 55, 79106 Freiburg, Germany

41

42 Keywords: SARS-CoV-2, COVID-19, CD8+ T-cell response, mRNA vaccination, 43 bnt162b2, Comirnaty, antiviral immunity, immunological memory, adaptive immune 44 response, $\mathrm{CD} 4+\mathrm{T}$ cell response, $\mathrm{B}$ cell response 


\section{Abstract}

SARS-CoV-2 spike mRNA vaccines ${ }^{1-3}$ mediate protection from severe disease as early as 10 days post prime vaccination ${ }^{3}$, when specific antibodies are hardly detectable and still lack neutralizing activity ${ }^{4-6}$. Vaccine-induced T cells, especially CD8+ T cells, may thus be the main mediators of protection at this early stage $e^{7,8}$. The details of antigenspecific CD8+ $\mathrm{T}$ cell induction after prime/boost vaccination, their comparison to naturally induced CD8+ $\mathrm{T}$ cell responses and their association with other arms of vaccine-induced adaptive immunity remain, however, incompletely understood. Here, we show on a single epitope level that both, a stable memory precursor pool of spikespecific CD8+ $T$ cells and fully functional spike-specific effector CD8+ $T$ cell populations, are vigorously mobilized as early as one week after prime vaccination when CD4+ T cell and spike-specific antibody responses are still weak and neutralizing antibodies are lacking. Boost vaccination after 3 weeks induced a full-fledged recall expansion generating highly differentiated CD8+ effector T cells, however, neither the functional capacity nor the memory precursor $T$ cell pool was affected. Compared to natural infection, vaccine-induced early memory $\mathrm{T}$ cells exhibited similar frequencies and functional capacities but a different subset distribution dominated by effector memory $T$ cells at the expense of self-renewing and multipotent central memory $T$ cells. Our results indicate that spike-specific CD8+ T cells may represent the major correlate of early protection after SARS-CoV-2 mRNA/bnt162b2 prime vaccination that precede other effector arms of vaccine-induced adaptive immunity and are stably maintained after boost vaccination. 
$\underline{\text { Introduction }}$

68 The SARS-CoV-2 vaccines by Biontech/Pfizer (bnt162b2) and Moderna (mRNA-1273) use mRNA-based technology to express stabilized SARS-CoV-2 spike protein ${ }^{1-3}$. Prime vaccination is followed by a boost 3-6 weeks later. Little is known about the precise nature of the immune response elicited by this formulation that has been licensed for the first time. The current vaccination campaign thus provides the unique opportunity to gain important insights into human CD8+ T cell biology in the context of prime/boost mRNA vaccination.

First data revealed that all arms of adaptive immunity such as neutralizing antibodies, virus-specific CD4+ T cells with $\mathrm{TH} 1$ polarization and IFN- $\gamma$-producing CD8+ T cells emerge after prime/boost vaccination ${ }^{4,5,9}$. However, early kinetics, differentiation trajectories, functional dynamics and differences to natural infection have not been addressed by these initial studies. Strikingly, the onset of mRNA vaccine-mediated protection has been observed as early as 10-12 days after the first dose ${ }^{3}$. During this early phase, $T$ cells and spike-specific antibodies are detectable ${ }^{7,8}$ while neutralizing antibodies first appear after boost ${ }^{4-6,10,11}$. These observations point towards a key role of vaccine-induced T cells in early protection after prime vaccination.

Previous studies focused on the analysis of the overall vaccine-elicited spike-reactive $\mathrm{T}$ cell responses, ${ }^{4,5,7,8,12}$ however, by this approach, the strength, dynamics and functional capacity of spike-specific CD8+ T cell responses are underestimated or even blurred in contrast to analyses performed on a single epitope level ${ }^{5}$. In this study, we performed continuous longitudinal analyses starting at baseline of prime vaccination until 3 months after boost on a single epitope level, to track the trajectories of bnt162b2 vaccine-elicited spike-specific CD8+ T cell responses. Additionally, we compared the kinetics and frequencies of the spike-specific CD8+ T cell response with spike-specific CD4+ T cells, B cells and antibodies and their neutralizing activity. 


\section{$\underline{\text { Results }}$}

Bnt162b2 vaccine-elicited epitope-specific CD8+ T cells: early induction, vigorous Teff response and robust establishment of memory precursors

To assess the dynamics of the spike-specific CD8+ T cell response following bnt162b2 vaccination, we longitudinally collected PBMCs and sera in a 3 to 4-day interval from 17 health care workers (Supplementary Table 1) that have not been previously infected with SARS-CoV-2, starting before prime until d84-86 after boost (Fig. 1A). We analyzed the induction of spike-specific CD8+ T cells targeting $A^{*} 01 / S_{865}$ and $A^{*} 02 / S_{269}$ epitopes in 5 individuals each (Extended Data Fig.1). Both epitopes are not highly conserved between SARS-CoV-2 and SARS-CoV-1, MERS or the common cold coronaviruses (229E, OC43, HKU1, NL63) (Extended Data Fig. 2A). Thus the detected spike-specific CD8+ $T$ cells indeed reflect a response to bnt162b2 vaccination. It is important to note that the epitopes are not affected by the sequence variations present in the variants of concern (VOC) B1.1.7, B1.351, P1 and B1.617 (Extended Data Fig. 2A). Ex vivo frequencies of $A^{*} 01 / S_{865}$ and $A^{*} 02 / S_{269}$-specific $C D 8+T$ cells were rather low after vaccination (Extended Data Fig. 2B). In order to increase the detection rate and to allow subsequent comprehensive profiling we performed $\mathrm{pMHCl}$-tetramer enrichment of $A^{*} 01 / S_{865}$ and $A^{*} 02 / S_{269}$-specific CD8+ T cells (Fig. 1B). We detected a rapid and substantial induction of spike epitope-specific CD8+ T cells in all tested donors at d68 that peaked 9-12 days post prime (dpp) (Fig. 1C). The strong activation of spike epitope-specific CD8+ T cells was also reflected by high expression of the activation marker CD38 and the proliferation marker Ki-67 as early as d6-8 in the majority of cells (Fig. 1D/E and Extended Data Fig. 2C). Boost vaccination led to a further increase of spike epitope-specific CD8+ T cell frequencies that peaked 5-6 days post boost (dpb) with a subsequent slow contraction phase reaching nearly pre-boost frequencies at about d84-86 post boost (Fig. 1C). Post-boost and post-prime expansion were 
119 accompanied by effector T (Teff) cell differentiation (high Ki-67, CD38, granzyme B,

120 PD-1, CD39, T-BET and TOX expression (Fig. 1D and Extended Data Fig. 2D/E).

121 However, t-SNE analysis of the concatenated expression analysis revealed that spike

122 epitope-specific CD8+ Teff cells are qualitatively different at the peak expansion

123 following boost (obtained at 5-6 dpb) compared to prime (obtained at 9-12 dpp) with a

124 more consolidated cytotoxic effector cell phenotype (increased T-BET, TOX, CD39

125 expressions) post boost (Extended Data Fig. 3A). This consolidated post-boost Teff

126 response is further supported by diffusion map analysis of the combined flow cytometry

127 data of vaccine-elicited spike epitope-specific CD8+ T cells (Fig. 1E and Extended

128 Data Fig. 3B/C). Specifically, diffusion map embedding revealed a continuous

129 relationship of the longitudinally collected spike-specific CD8+ T cells following prime

130 (depicted in reddish colors)/boost (depicted in grey shaded colors) indicating a directed

131 trajectory of the Teff response from prime to boost vaccination (Fig. 1E and Extended

132 Data Fig. 3C). Along the indicated trajectory, spike-specific CD8+ T cells exhibited

133 highest expression of PD-1, TOX, T-BET and CD38 after boost that remained at high

134 levels, indicating profound activation and progressing differentiation post-boost (Fig.

$1351 \mathrm{E})$.

136 Next to the emergence of an effective Teff response after bnt162b2 vaccination, we

137 also assessed the induction of a spike epitope-specific memory precursor CD8+ T cell

138 (TMP) pool. TMP cells are characterized by CD127, BCL-2 and TCF-1 expression and

139 are relevant for maintaining the CD8+ T cell response ${ }^{13,14}$. Roughly $30 \%$ of $A^{*} 01 / S_{865-}$

140 and $A^{*} 02 / S_{269}$-specific CD8+ $T$ cells expressed CD127 after prime followed by a

141 transient reduction and subsequent strong increase after boost (Fig.1F and Extended

142 Data Fig. 4A). Expression dynamics of TCF-1 (Fig. 4G and Extended Data Fig. 4B)

143 and BCL-2 (Extended Data Fig. 4C) were similar to CD127. However, the overall

144 frequency of CD127+ (Fig.1F) and TCF-1+ (Fig.1G) spike epitope-specific CD8+ T 
145 cells remained constant indicating a stable memory precursor pool of spike-specific

146 CD8+ $T$ cells induced already early after prime and being unaffected by boost

147 vaccination. Taken together, bnt162b2 vaccination vigorously induces a lasting spike-

148 specific CD8+ T cell response rapidly after prime.

150 Proper and conserved functional capacities of circulating spike-specific CD8+ T cells

151 following prime and boost vaccination

152 To assess spike-specific CD8+ $T$ cell function after prime/boost vaccination, we

153 analyzed expansion capacity, cytokine production and degranulation after peptide-

154 specific expansion (Fig. 2A/B). After two weeks of in vitro expansion, we detected

155 higher frequencies of $A^{*} 01 / S_{865}$ and $A^{*} 02 / S_{269}$-specific CD8+ $T$ cells after boost

156 compared to prime vaccination (Fig. 2C). However, the expansion index, a measure

157 taking the input number of virus-specific CD8+ T cells into account was comparable

158 for spike epitope-specific CD8+ T cells after prime and boost vaccination (Fig. 2D).

159 Thus, the increased frequencies of spike epitope-specific CD8+ T cells after peptide-

160 specific expansion most probably result from the increased ex vivo frequencies after

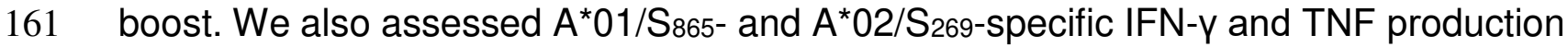

162 (Fig. 2E-G) and degranulation (indicated by CD107a expression; Fig. 2H) in relation to

163 the frequency of spike epitope-specific CD8+ T cells after expansion as a measure of

164 the effector function per cell. We observed proper effector capacity of circulating spike

165 epitope-specific CD8+ T cells obtained as early as 6-8 dpp. Similar to the expansion

166 capacity, cytokine production and degranulation capacity remained nearly stable after

167 boost compared to prime. Hence, functionally competent spike-specific CD8+ T cells

168 targeting different epitopes are substantially induced early after prime, and subsequent

169 boost vaccination does not further increase their functional capacities. 
171 Delayed appearance of circulating spike-specific CD4+ T cells, B cells and antibodies

172 Next, we compared kinetics and frequencies of spike-specific CD8+ T cells with spike

173 epitope-specific CD4+ T cells, B cells and antibodies with neutralizing activity. For this,

174 we first longitudinally assessed circulating spike-specific CD4+ T cells targeting 175 DRB1*15:01/S 236 (Extended Data Fig. 5A) following prime and boost vaccination in 8 176 individuals (Supplementary Table 1). The selected DRB1*15:01/S 236 epitope is rather 177 unique for SARS-CoV-2 in comparison to SARS-CoV-1, MERS or common cold 178 coronaviruses (229E, OC43, HKU1, NL63) and conserved in circulating SARS-CoV-2 179 variants (B.1, B.1.1.7, P.1, B.1.617) except for VOC B.1.351 (Extended Data Fig. 5B). 180 As expected for virus-specific CD4 T cells, the frequencies of DRB1*15:01/S $236^{-}$ 181 specific CD4+ T cells were lower compared to CD8 responses but detectable after 182 pMHCIl tetramer-based enrichment (Fig. 3A). At baseline (Fig. 3A) and in historic 183 control (HC) samples (banked before August 2019) (Extended Data Fig. 5C), spike184 specific CD4+ T cells were detectable with a primarily naïve phenotype (Extended Data 185 Fig. 5C and D, respectively) reflecting the presence of antigen-unexperienced 186 precursors. Following vaccination, the proportion of circulating spike epitope-specific 187 CD4+ T cells with a naïve phenotype decreased suggesting vaccine-induced activation 188 of the precursor cells (Extended Data Fig. 5D). However, compared to the vaccine189 elicited CD8+ T cell response, we observed a lower mobilization of circulating spike 190 epitope-specific CD4+ T cells indicated by a limited increase of frequencies (Fig. 3A) 191 and a smaller percentage of activated ICOS+CD38++ or Ki-67+ subsets (Fig. 3B and 192 Extended Data Fig. 5E) even after boost vaccination. The majority of activated 193 (ICOS+CD38++ or Ki-67+) circulating DRB1*15:01/S $236-$ specific CD4+ T cells 194 exhibited a TH1 phenotype (Fig. 3C). In line with this observation, vaccine-induced 195 spike epitope-specific CD4+ T cell response displayed a $\mathrm{TH} 1$ rather than a TFH 196 phenotype (Extended Data Fig. 5F). 
197 Next, we assessed the kinetics of the vaccine-induced humoral response. The

198 distribution of peripheral B cell subpopulations was stable throughout prime/boost

199 vaccination, with the exception of a progressively slight increase in antibody-secreting 200 cells (Extended Data Fig. 6A/B). An increase in the frequency of CD95+ B cells was 201 observed shortly after boost indicating ongoing B cell activation via CD40-mediated T 202 cell help and/or B cell receptor activation within secondary lymphoid organs (Extended 203 Data Fig. 6B $)^{15}$. In line with the appearance of activated B cells in the periphery, we 204 observed a progressive maturation of the serum antibody response with S1-specific IgM present after prime whereas S1-specific $\lg G$ reasonably detectable after boost (Fig. 3D and Extended Data Fig. 6C) coinciding with a high neutralization capacity in SARS-CoV-2 plaque reduction assays (Fig. 3E). More precisely, SARS-CoV-2 B.1, 208 circulating in early 2020, and SARS-CoV-2 VOC B1.1.7, emerging in the UK in late 209 2020, were equally well neutralized by post-boost sera whereas the neutralization activity against SARS-CoV-2 VOC B1.351, a variant emerging in Brazil in late 2020,

211 was reduced approximately by a factor of 5 (Fig. 3E). Neutralization capacity of post212 boost sera was clearly elevated compared to time point-matched mild infection 213 (Extended Data Fig. 6D). To test the frequency of spike-specific B cells we applied S1 and receptor-binding domain (RBD) tetramers ex vivo (Fig. 3F). In line with the progressive maturation of the antibody response, S1- and RBD-specific B cells largely remained below the detection limit until the first week post boost vaccination (Fig. 3G).

217 The delayed appearance of circulating S1-specific B cells was confirmed by polyclonal 218 restimulation in vitro (Fig. $3 \mathrm{H}$ ) showing a limited presence of class-switched B cells 219 capable to produce S1-specific IgG before boost. S1-specific B cells were largely 220 unswitched after prime (Fig. 3I), also reflected by S1-specific IgM production upon 221 polyclonal restimulation in vitro (Extended Data Fig. 6C), and acquired a memory 222 phenotype after boost vaccination (Fig. 3I and Extended Data Fig. 6E). In addition, 
223 after boost vaccination, S1-specific B cells showed increased transferrin receptor 224 (CD71) and CD95 expression (Fig 3I and Extended Data Fig. 6E) indicating their germinal center origin ${ }^{16}$. Hence, bnt162b2 vaccination efficiently elicits a protective humoral immune response, composed of both antibody secreting cells and antigenspecific memory cells that are mobilized to the periphery after boost.

Differences of circulating spike-specific CD8+ early memory CD8+ T cells after boost vaccination and natural infection

In a last set of experiments, we compared vaccine-elicited $A^{*} 01 / S_{865}$ and $A^{*} 02 / S_{269-}$ specific early memory CD8+ T cells with time point-matched T cells induced by natural infection. Spike epitope-specific CD8+ T cell frequencies and CD127 expression, a 234 hallmark of lasting $T$ cells, were similar at all time points analyzed, with a slight

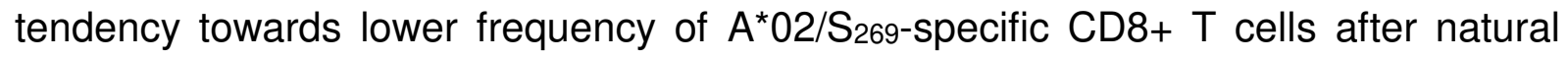
infection (Fig. 4A and Extended Data Fig. 7A). We also observed differences in T cell memory subset distribution (Extended Data Fig. 7B) of spike-specific early memory

238 CD8+ T cells with higher fractions of more early differentiated subsets, e.g. early 239 differentiated (TED) and central memory T cells (TCM) after natural infection. In 240 contrast, more effector memory $1 \mathrm{~T}$ cells (TEM1) were detectable after vaccination 241 (Fig. 4B). Of note, spike-specific effector memory 2 and 3 T cells (TEM2, TEM3) and 242 terminally differentiated effector memory T cells expressing CD45RA (TEMRA) were 243 hardly detectable in the circulation neither after vaccination nor after natural infection 244 (Extended Data Fig. 7C). In addition, t-SNE analysis of concatenated expression data 245 (including differentiation, activation, anti-apoptotic marker molecules) further supports 246 qualitative differences of spike-specific CD8+ T cells obtained from the early memory 247 phase ( $>80 \mathrm{dpb} / \mathrm{dps})$ after vaccination compared to natural infection. Indeed, we 248 observed a higher expression of CCR7, BCL-2 and TCF-1 after natural infection, (Fig. 
249 4C and Extended Data Fig. 8A) suggesting a higher capacity of homeostatic 250 maintenance of the spike-specific early memory CD8+ T cell population. Both, t-SNE 251 analysis and manual gating demonstrate a higher and prolonged CD38 expression on 252 spike epitope-specific CD8+ T cells after natural infection possibly due to different 253 dynamics of antigen recognition (Fig. 4C and Extended Data Fig. 8B). However, 254 vaccine- and natural infection-associated expansion capacity and cytokine production 255 of spike-specific CD8+ T cells are similar (Fig. 4D and Extended Data Fig. 8C). Hence, 256 compared to natural infection, vaccine-associated spike-specific early memory CD8+ 257 T cell populations exhibit similar functional capacities but a different subset distribution 258 dominated by effector memory T cells at the expense of self-renewing and multipotent, 259 early differentiated subsets. 
Discussion

261 Our comprehensive analysis of vaccine induced immunity revealed a rapid and strong 262 induction of CD8+ T cells targeting two spike epitopes as early as one week after prime 263 vaccination in all vaccinees at a time point when neutralizing antibodies are still lacking. 264 Importantly, these immediately mobilized spike-specific CD8+ T cells are fully 265 functional and able to mount a proficient recall response evoked e.g. by the boost 266 vaccination. These findings clearly suggest a major protective role of vaccine-elicited 267 CD8+ T cells early after first dose vaccination. In agreement with this notion, the protective effect observed for mRNA vaccines starts at 10-12 $\mathrm{dpp}^{2,3}$ and thus coincides with the first peak of spike-specific CD8+ T cells. A major protective capacity of virusspecific CD8 T cells was also observed in natural SARS-CoV-2 infection, since e.g.

271 CD8+ T cells compensate for impaired humoral immunity in COVID-19 patients with hematologic cancer ${ }^{17}$. The approved SARS-CoV-2 mRNA vaccine design includes a boost after 3-6 weeks ${ }^{2,3}$.

274 Our data demonstrate that the boost after 3 weeks hits the spike-specific CD8+ T cells 275 at the beginning of the contraction phase of the primary response and results in a 276 robust recall expansion of spike-specific CD8+ T cells. The generation of a fortified Teff 277 response, however, neither leads to an improved functional capacity nor to an 278 increased frequency of memory precursor T cells relevant for maintaining the CD8+ T 279 cell response. These observations may indicate that a robust spike-specific CD8+ T 280 cell response is already elicited after prime with only transient effects of boosting after 2813 weeks.

282 In contrast to CD8+ T cells, efficient mobilization of a humoral immune response comprising neutralizing antibodies and antigen-specific memory B cells to the periphery was first detectable after boost. This is in line with previous reports $4,11,12,18$ and most probably represents maturation of the response that is initially localized in 
secondary lymphoid organs (SLO) ${ }^{19}$ and subsequently released to the circulation.

287 However, how the boost affects this SLO response requires further investigation. After boost, highly cross-neutralizing antibodies are present in the sera clearly adding a major correlate of protection on top of the early-mobilized spike-specific CD8+ T cell 290 response.

291 A recent study has highlighted the important role of spike-reactive CD4+ T cells in 292 coordinating the humoral and CD8+ T cell response ${ }^{8}$. Similar to CD8+ T cells, spike293 reactive CD4+ $T$ cells are also mobilized early after prime ${ }^{8,18}$. We also observed an early but moderate appearance of spike-specific CD4+ T cells with a predominance of a $\mathrm{TH} 1$ over a TFH phenotype in the periphery. While a dominant $\mathrm{TH} 1$ polarization has also been reported by others ${ }^{4}$, spike-reactive TFH cells may also be crucial for coordinating humoral and cellular immune responses upon mRNA vaccination ${ }^{8}$. Further studies need to clarify whether the parallel TH1 - and TFH-directed coordination is restricted by different CD4+ T cell epitopes or clonotypes, by pre-existing crossreactive CD4+ T cells or by CD4+ $\mathrm{T}$ cell localization (periphery versus SLO). Unlike spike-specific CD8+ T cells, only a limited recall expansion was detectable for 302 circulating spike-specific CD4+ T cells, supporting their coordinating role after mRNA vaccination.

Hallmark of an efficient vaccine is the induction of lasting immune memory. In parallel to the early appearance of circulating spike-specific CD8+ T cells, a stable memory precursor pool is established that develops after boost into a fully functional early memory CD8+ T cell response. Of note, the functional capacity of spike-specific early memory CD8+ $\mathrm{T}$ cells is similar after vaccination and natural infection up to three months post boost/symptom onset. Compared to natural infection, however, the early memory pool of spike-specific CD8+ T cells after vaccination contains a higher 311 proportion of effector memory 1 (TEM1) T cells at the expense of central memory 
312 (TCM) and early differentiated (TED) T cells. Of note, the latter maintain long-term memory e.g. after yellow fever virus (YFV) vaccination ${ }^{20}$. In line with this, expression

314 of CCR7, BCL-2 and TCF-1 is lower after vaccination compared to infection. These

315 observations may hint towards a restricted self-renewal and maintenance of spike316 specific CD8+ T cells after vaccination compared to infection. This difference may be

317 caused by differential duration and location of antigen contact after vaccination versus

318 infection ${ }^{21,22}$. Indeed, we observed a lower CD38 expression on early memory spike-

319 specific CD8+ T cells after vaccination possibly indicating limited antigen recognition

320 compared to natural infection ${ }^{4,23}$. So far, we have followed our cohort for only 3 months

321 after boost; follow-up studies including larger cohorts of vaccinees and SARS-CoV-2

322 convalescent donors should comparatively assess longevity of CD8+ T cell immunity.

323 In addition, our study was limited to circulating spike-specific adaptive immunity, not

324 addressing local immunity at the viral entry site, the respiratory tract.

325 In sum, our data clearly highlight that a robust, stable and fully functional spike-specific

326 CD8+ T cell response is rapidly mobilized already after prime vaccination and is able 327 to patrol the periphery for SARS-CoV-2 at least within the first months. These 328 observations do not only provide insides into the protective mechanisms underlying bnt162b2 vaccination, but are also of potential relevance for the development of novel

330 vaccination strategies against emerging pathogens and cancer. 
Methods

332 Study cohort

33317 health care workers that received a prime and boost vaccination with the mRNA 334 vaccine bnt162b2/Comirnaty, 24 convalescent individuals following a mild course of 335 SARS-CoV-2 infection and historic controls (sampled before August 2019) of 8 healthy 336 individuals were recruited at the Freiburg University Medical Center, Germany. A mild 337 course of infection was characterized by clinical symptoms without respiratory 338 insufficiency. SARS-CoV-2 infection was confirmed by positive PCR testing from 339 oropharyngeal swab and/or SARS-CoV-2 spike IgG positive antibody testing. Donor 340 characteristics are summarized in Supplementary Table 1. HLA-typing was performed 341 by next-generation sequencing and is listed in Supplementary Table 1.

\section{Ethics}

344 Written informed consent was obtained from all participants and the study was 345 conducted according to federal guidelines, local ethics committee regulations (Albert346 Ludwigs-Universität, Freiburg, Germany; vote \#: 322/20, \#21-1135 and 315/20) and 347 the Declaration of Helsinki (1975).

PBMC isolation

350 Venous blood samples were collected in EDTA-anticoagulated tubes. Peripheral blood 351 mononuclear cells (PBMCs) were isolated with lymphocyte separation medium density 352 gradients (Pancoll separation medium, PAN Biotech GmbH; Aidenbach, Germany) and stored at $-80^{\circ} \mathrm{C}$. Frozen PBMCs were thawed in complete medium (RPMI 1640 354 supplemented with $10 \%$ fetal calf serum, $1 \%$ penicillin/streptomycin, and $1.5 \%$ HEPES 355 buffer $1 \mathrm{~mol} / \mathrm{L}$ (all additives from Thermo Scientific (Waltham, MA)) containing $50 \mathrm{U} / \mathrm{mL}$ 356 benzonase (Sigma, Germany). 
Sequence alignment

358 Sequence homology analyses were performed in Geneious Prime 2020.0.3

359 (https://www.geneious.com/) using Clustal Omega 1.2.2 alignment with default settings

36024 . Reference genomes of human coronaviruses 229E (NC_002645), HKU1

361 (NC_006577), NL63 (NC_005831), OC43 (NC_006213), MERS (NC_019843), SARS-

362 CoV-1 (NC_004718) and SARS-CoV-2 (MN908947.3) were downloaded from NCBI

363 database. Spike proteins of human coronaviruses were aligned according to their

364 homology (amino acid level). Analyzed Spike SARS-CoV-2 epitopes were then

365 mapped to the corresponding protein alignment. Correspondingly mutation analyses

366 were performed with the spike protein of variants of concern (VOC) B.1.1.7, B1.351,

367 P.1 and B.1.617.

Peptides and tetramers for $T$ cell analysis

370 Peptides were synthesized with an unmodified N-terminus and an amidated C-

371 terminus with standard Fmoc chemistry and a purity of $>70 \%$ (Genaxxon Bioscience,

372 Germany). Peptide was loaded on HLA class I easYmers® (immunAware, Denmark)

373 according to manufacturer's instructions $\left(A^{*} 01 /\right.$ S865 LTDEMIAQY and $A^{*} 02 / S_{269}$

374 YLQPRTFLL). SARS-CoV-2 peptide-loaded HLA class I tetramers were produced by

375 conjugation of biotinylated peptide-loaded HLA class I easYmers ${ }^{\circledR}$ with phycoerythrin

376 (PE)-conjugated streptavidin (Agilent, USA) according to the manufacturer's

377 instructions. A SARS-CoV-2-specific HLA class II custom tetramer (DRB1*15:01/S236

378 TRFQTLLALHRSYLT) was obtained from (MBL, USA).

380 In vitro expansion of spike-specific CD8+ T cells and assessment of effector function

$3811^{\star} 10^{6}$ PBMCs were stimulated with $A^{\star} 01 / S_{865}$ or $A^{\star} 02 / S_{269}$ spike-specific peptides

$382(5 \mu \mathrm{M})$ and anti-CD28 $\mathrm{mAb}(0.5 \mu \mathrm{g} / \mathrm{mL}$, BD) and expanded for 14 days in complete 
383 RPMI culture medium containing rlL2 (20 IU $/ \mathrm{mL}$, Stemcell Technologies). Intracellular 384 cytokine production and degranulation was assessed with spike-specific peptides (15 $\mu \mathrm{M})$ in the presence of anti-CD107a (H4A3, 1:100) (BD Bioscience, Germany) for $1 \mathrm{~h}$ at $37^{\circ} \mathrm{C}$. Afterwards, brefeldin A (GolgiPlug, $0.5 \mu \mathrm{L} / \mathrm{mL}$ ) and monensin (GolgiStop, 0.5 $\mu \mathrm{L} / \mathrm{mL}$ ) (all BD Biosciences, Germany) were added for additional $5 \mathrm{~h}$, followed by surface and intracellular staining. The expansion capacity was calculated based on peptide-loaded HLA class I tetramer staining as described before ${ }^{25}$.

Magnetic bead-based enrichment of spike-specific CD8+ T cells

Spike-specific CD8+ T cells were enriched as previously described ${ }^{26}$. Briefly, $1-2^{*} 10^{7}$

393 PBMCs (with an average of $15.7 \%$ CD8+ T cells) were labelled with PE-coupled 394 peptide-loaded HLA class I tetramers for 30 min. Enrichment was then performed using anti-PE beads with MACS technology (Miltenyi Biotec, Germany) according to the manufacturer's instructions. Subsequently, enriched spike-specific CD8+ T cells were analyzed by multiparametric flow cytometry and frequencies of spike-specific CD8+ T cells were calculated as described before ${ }^{26}$. Only enriched samples with $\geq 5$ spikespecific CD8 T cells were included in further analyses, resulting in a detection limit of $5^{\star} 10^{-6}$.

Magnetic bead-based enrichment of spike-specific CD4+ T cells

403 Enrichment of spike-specific CD4+ T cells was adapted from the method described 404 previously ${ }^{26}$. In brief, $1.5-2^{*} 10^{7}$ PBMCs of DRB ${ }^{*} 15: 01$-positive donors were labelled with PE-coupled peptide-loaded MHC class II tetramers for 40 min. $5 \mu$ l were taken from $1000 \mu \mathrm{l}$ pre-enriched sample (1:200) and used for subsequent flow cytometric

407 staining. Subsequent enrichment was performed with anti-PE beads using MACS 408 technology (Miltenyi Biotec, Germany) according to the manufacturer's protocol. 
409 Enriched spike-specific CD4+ T cells and the pre-enriched sample were used for flow

410 cytometric staining. The complete pre-enriched and enriched samples were recorded.

411 Only enriched samples with $\geq 5$ spike-specific CD4+ T cells were included in further

412 analyses. The frequency of spike-specific CD4+ T cells was calculated as follows:

413 Absolute number of spike-specific CD4+ $T$ cells (enriched sample) divided by the

414 absolute number of CD4+ T cells (pre-enriched sample) $\times 200$. The detection limit as

415 a frequency was calculated as follows: 5 spike-specific CD4+ T cells (enriched sample)

416 divided by the mean number of CD4+ $\mathrm{T}$ cells (pre-enriched sample) throughout all

417 tested donors $\times 200$.

Multiparametric flow cytometry for $T$ cell analysis

420 The following antibodies were used for multiparametric flow cytometry: anti-CCR7-PE-

421 CF594 (150503, 1:50), anti-CCR7-BUV395 (3D12, 1:25), anti-CD4-BV786 (L200,

1:200), anti-CD8-BUV395 (RPA-T8, 1:400), anti-CD8-BUV510 (SK1, 1:100), anti-CD8APC (SK-1, 1:200), anti-CD11a-BV510 (HI111, 1:25), anti-CD28-BV421 (CD28.2, 1:100), anti-CD38-APC-R700 (HIT2, 1:400), anti-CD38-BUV737 (HB7, 1:200), antiCD39-BV650 (TU66, 33:1), anti-CD45RA-BUV496 (HI100, 1:800), anti-CD45RABUV737 (HI100, 1:200), anti-CD69-BUV395 (FN50, 1:50), anti-CD107a-APC (H4A3, 1:100), anti-CD127-BUV737 (HIL-7R-M21, 1:50), anti-CD127-BV421 (HIL-7R-M21, 3:100), anti-EOMES-PerCP-eF710 (WD1928, 1:50), anti-Granzyme B-PE-CF594

429 (GB11, 1:100), anti-ICOS-BV711 (DX29, 1:100), anti-IFN-y-FITC (25723.11, 1:8), anti430 IL-21-PE (3A3-N2.1, 1:25), anti-PD-1-BV605 (EH12.1, 1:50), anti-PD-1-PE-Cy7 431 (EH12.2H7, 1:200), anti-PD-1-BV786 (EH12.1, 1013122, 3:100), anti-T-BET-PE432 CF594 (O4-46,93533305, 3:100), anti-TNF-PE-Cy7 (Mab11, 1:400) (BD Biosciences, 433 Germany), anti-BCL-2-BV421 (100, 1:200), anti-CCR7-BV785 (G043H7, 1:50), anti434 CD4-AlexaFluor700 (RPA-T4, 300526, 1:200), anti-CD25-BV650 (BC96, 1:33), anti- 
435 CD57-BV605 (QA17A04, 1:100), anti-CD127-BV605 (A019D5, 3:100), anti-CXCR3-

436 PerCP-Cy5.5 (G025H7, 1:33), anti-CXCR3-BV510 (G025H7, 3:100), anti-CXCR5-

437 BV421 (J252D4, 1:100), anti-IL-2-PerCP-Cy5.5 (MQ1-17H12, 1:100), anti-Ki-67-

438 BV711 (Ki-67, 1:200), anti-Ki-67-PE-Cy7 (Ki-67, 1:200) (BioLegend, UK), anti-TCF-1-

439 AlexaFluor488 (C63D9, 1:100) (Cell Signaling, Germany), anti-CD14-APC-eFluor780

440 (61D3, 1:400), anti-CD19-APC-eFluor780 (HIB19, 1:400), anti-CD27-FITC (0323,

441 1:100), anti-KLRG1-BV711 (13F12F2, 1:50), anti-T-BET-PE-Cy7 (4B10, 1:200), anti-

442 TOX-eFluor660 (TRX10, 1:100) (Thermo Fisher, Germany), anti-CD45RA-PerCP-

443 Cy5.5 (HI100, 3:100) (Invitrogen, Germany). For live/dead discrimination a fixable

444 Viability Dye (APC-eFluor780 1:200, 1:400) (Thermo Fisher, Germany) or ViaProbe

445 (7-AAD, 1:33) (BD Biosciences, Germany)) was used. FoxP3/Transcription Factor

446 Staining Buffer Set (Thermo Fisher, Germany) and Fixation/Permeabilization Solution

447 Kit (BD Biosciences, Germany) were used according to the manufacturer's protocol to

448 stain for intranuclear and cytoplasmic molecules, respectively. After fixation of cells in

$4492 \%$ paraformaldehyde (PFA, Sigma, Germany), analyses were performed on

450 FACSCanto II, LSRFortessa (BD, Germany) or CytoFLEX (Beckman Coulter). Data 451 were analyzed with FlowJo 10 (Treestar, USA).

453 Dimensional reduction of multiparametric flow cytometry data

454 Dimensionality reduction of multiparametric flow cytometry data was done with $\mathrm{R}$ 455 version 4.0.2 using the Bioconductor (release (3.11)) CATALYST package23. The 456 analyses were performed on gated virus-specific CD8+ T cells including the markers 457 CD69, CD45RA, BCL-2, PD1, CD25, Ki-67, TCF-1, Eomes, CCR7, Tbet, TOX, CD38. 458 For Figure 4 t-SNE analysis the markers CD69, Eomes and Ki-67 were excluded. 459 Downsampling of cells to 100 or 200 cells (t-SNE Figure 4 and Extended data 3, 460 diffusion maps Figure 1 and Extended data 3) was performed before dimensionality 
461 reduction to facilitate the visualization of different samples. Marker intensities were

462 transformed by arcsinh (inverse hyperbolic sine) with a cofactor of 150 . Dimensionality 463 reduction on the transformed data was achieved by t-SNE and diffusion map 464 visualization.

S1- and RBD-tetramerization for $B$ cell analysis

467 A biotinylated form of recombinant S1 and RBD proteins (BioLegend, UK) were 468 tetramerized by addition of PE-conjugated or BV421-conjugated streptavidin 469 (BioLegend, UK) and used for B cell tetramer staining assays. Briefly, the Streptavidin470 PE or Streptavidin-BV21 were added in an amount that equals $1 / 5$ of the monomer 471 substrate amount. The Streptavidin was added in 5 equal portions to the monomer and 472 incubated each time at $4^{\circ} \mathrm{C}$ for 20 min on a shaker. The tetramers were filled up to 100 $473 \mu \mathrm{l}$ with $0.1 \% \mathrm{BSA}$ in PBS and stored at $4^{\circ} \mathrm{C}$.

Multiparametric flow cytometry for B cell analysis

476 Phenotype of vaccinated subjects' PBMCs was determined by flow cytometry (Cytek 477 Aurora, Cytek) with the following antibodies: anti-CD20-BV510 (2H7, 1:80), anti-IgM478 BV605 (MHM-88, 1:200), anti-CD71-FITC (CY1G4, 1:1000), anti-CD95-PE-Dazzle594 479 (DX2, 1:50), anti-CD24-FITC (ML5, 1:100), anti-CD38-PE-Cy7 (HB-7, 1:300), anti480 BAFF-R-AF647 (11C1, 1:100), anti-CD19-APC-Cy7 (HIB19, 1:150) (BioLegend, UK); 481 anti-IgG-BV650 (G18-145, 1:600), anti-CD27-BV786 (L128, 1:100), anti-CD69-BV480 482 (FN50, 1:200) (BD Biosciences, Germany); anti-lgA-PerCP (polyclonal, 1:200) 483 (Jackson ImmunoResearch, UK); anti-CD3-Super Bright 436 (OKT3, 1:200), anti484 CD33-Super Bright 436 (WM-53, 1:50), anti-lgD-PerCP-eFluor 710 (IA6-2, 1:200) 485 (Invitrogen, Germany). Dead cell exclusion was performed by Zombie NIR Fixable 486 Viability Kit (Biolegend, UK, 1:800). 
488 PBMCs of vaccinated subjects and patients with a history of SARS-CoV-2 infection 489 were plated at $0.5^{\star} 10^{6}$ cells $/ \mathrm{ml}$ and polyclonally stimulated for 9 days with thiol-modified

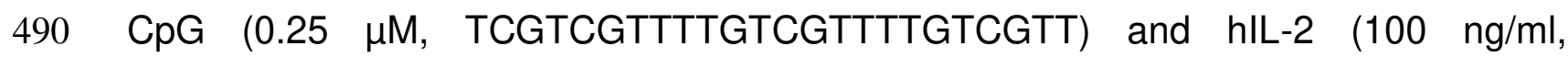
491 Immunotools). At day 9, the supernatants of the in vitro culture were cleared from 492 debris by centrifugation and used to determine the presence of SARS-CoV-2 Spike493 specific IgG antibodies (Anti-SARS-CoV-2-QuantiVac-ELISA (IgG), Euroimmun) 494 according to the manufacturer's instructions. To detect S1 specific IgM, supernatant of 495 the in vitro culture and serum of vaccinated subjects was incubated on a S1 pre-coated 496 plate (Anti-SARS-COV-2, Euroimmun). Bound IgM was detected with alkaline 497 phosphatase-conjugated anti-human IgM (Jackson ImmunoResearch), and developed 498 with p-nitrophenyl phosphate (Sigma-Aldrich) in DEA buffer.

Serum IgG determination

SARS-CoV-2-specific antibodies were determined by Anti-SARS-CoV-2-QuantiVac-

502 ELISA (IgG) from Euroimmun detecting anti-SARS-CoV-2 Spike IgG (anti-SARS-CoV$5032 \mathrm{~S}$ IgG; $<35,2 \mathrm{BAU} / \mathrm{ml}$ : negative, $\geq 35,2 \mathrm{BAU} / \mathrm{ml}$ : positive) according to the 504 manufacturer's instructions.

Neutralization assay

507 Samples of vaccinated and convalescent individuals were tested in a plaque reduction 508 neutralization assay. Briefly, VeroE6 cells were seeded in 12-well plates at a density 509 of $2.8 * 10^{5}$ cells/well $24 \mathrm{~h}$ prior to infection. Serum samples were diluted at ratios of $5101: 16,1: 32,1: 64,1: 128,1: 256,1: 512$, and 1:1024 in $50 \mu \mathrm{L}$ PBS total volume. For each 511 sample, one negative control was included (PBS without serum). Diluted sera and 512 negative controls were subsequently mixed with 90 plaque forming units (PFU) of 
513 authentic SARS-CoV-2 (either B.1, B.1.1.7 or B.1.351 variant) in $50 \mu$ PBS (1600

$514 \mathrm{PFU} / \mathrm{mL}$ ) resulting in final sera dilution ratios of $1: 32,1: 64,1: 128,1: 256,1: 512,1: 1024$, 515 and 1:2048. Following incubation at RT for $1 \mathrm{~h}, 400 \mu \mathrm{L}$ PBS was added to each sample 516 and the mixture was subsequently used to infect VeroE6 cells. After $1.5 \mathrm{~h}$ of incubation 517 at RT, inoculum was removed and the cells were overlaid with $0.6 \%$ Oxoid-agar in 518 DMEM, 20 mM HEPES (pH 7.4), 0.1\% $\mathrm{NaHCO}_{3}, 1 \%$ BSA and 0.01\% DEAE-Dextran. 519 Cells were fixed $72 \mathrm{~h}$ post-infection using $4 \%$ formaldehyde for 30 minutes and stained 520 with $1 \%$ crystal violet upon removal of the agar overlay. PFU were counted manually. 521 Plaques counted for serum-treated wells were compared to the average number of 522 plaques in the untreated negative controls, which were set to $100 \%$. The plaque523 reduction neutralization titer 50 (PRNT50) was calculated using a linear regression 524 model in GraphPad Prism 9 (GraphPad Prism Software, USA).

526 Statistics

527 Statistical analysis was performed with GraphPad Prism 9 (GraphPad Prism Software, 528 USA). Statistical significance was assessed by 2way ANOVA including Tukey's 529 multiple comparisons test and Spearman correlation. $\left({ }^{*} \mathrm{P}<0.05 ;{ }^{* \star} \mathrm{P}<0.01 ;{ }^{* \star} \mathrm{P}<0.001\right.$; $530 * * * * P<0.0001)$. 
532 Raw data is available upon reasonable request. Further supporting data are available

533 from the corresponding authors upon reasonable request. All requests for raw and

534 analyzed data and materials will be reviewed by the corresponding authors to verify if

535 the request is subject to any intellectual property or confidentiality obligations. Patient-

536 related data not included in the paper were generated as part of clinical examination

537 and may be subject to patient confidentiality. Any data and materials that can be shared

538 will be released via a Material Transfer Agreement. Source data are provided with this

539 paper. Reference viral sequences SARS-CoV-2

(MN908947.3)

540 https://www.ncbi.nlm.nih.gov/nuccore/MN908947,

229E (NC_002645)

541 https://www.ncbi.nlm.nih.gov/nuccore/NC_002645, HKU1 (NC_006577)

542 https://www.ncbi.nlm.nih.gov/nuccore/NC_006577, NL63 (NC_005831)

543 https://www.ncbi.nlm.nih.gov/nuccore/NC_005831, OC43 (NC_006213)

544 https://www.ncbi.nlm.nih.gov/nuccore/NC_006213, MERS (NC_019843)

545 https://www.ncbi.nlm.nih.gov/nuccore/NC_019843, SARS-CoV-1 (NC_004718)

546 https://www.ncbi.nlm.nih.gov/nuccore/NC_004718) were downloaded from the NCBI 547 database (https://www.ncbi.nlm.nih.gov/).

Code availability statement

$550 \mathrm{R}$ code to reproduce the analyses of multiparametric flow-cytometry data is available 551 at https://github.com/sagar161286/SARSCoV2_specific_CD8_Tcells.

\section{Acknowledgements}

554 We thank all donors for participating in the current study and the FREEZE-Biobank

555 Center for biobanking (Freiburg University Medical Center) and the Medical Faculty for 556 support. The study was funded by the Federal Ministry of Education and Research 
557 (grant number $01 \mathrm{KI} 2077$ to G.K., M.H., M.S. and R.T.) and by COVID-19 research 558 grants of the Ministry of Science, Research and Art, State of Baden-Wuerttemberg 559 (COVID-19/AZ.: AZ33-7533-6-10/89/8 to C.N.-H and B.B.). The presented work was 560 also supported by CRC/TRR 179-Project 01 and CRC 1160-Project A02 (to R.T.), 561 CRC/TRR 179-Project 02 and CRC 1160-Project A06 (to C.N.-H.), CRC 1160-Project 562 B02 (M.R.), CRC/TRR 179-Project 04 (to T.B.), CRC/TRR 179-Project 20 and CRC 1160-Project A02 (to M.H.), CRC/TRR 179-Project 21, CRC 1160-Project A03 and BE$5645496 / 5-1$ (to B.B.) of the German Research Foundation (DFG; TRR 179 project no. 272983813; CRC 1160 project no. 256073931). M.H. was supported by a Margarete von Wrangell fellowship (State of Baden-Wuerttemberg). T.B. was supported by the

Berta-Ottenstein Programme, Faculty of Medicine, University of Freiburg. H.L. was supported by the IMM-PACT-Programme for Clinician Scientists, Department of Medicine II, Medical Center - University of Freiburg and Faculty of Medicine, University of Freiburg. The funding body had no role in the decision to write or submit the 571 manuscript.

Authors Contributions

V.O., H.L., J.K. and I.S. planned, performed and analyzed experiments with the help of K.C., S.G., I.J., J.S., K.W., S.M., B.C., K.B., J.F., F.T., A.J., K.Z. S. and O.S.. H.L., J.L.M., B.B., C.W. and S.R. were responsible for donor recruitment. F.E. performed 577 four-digit HLA typing by next-generation sequencing. T.B., G.K., M.S., M.R., R.T., 578 C.N.-H and M.H. designed the study and contributed to experimental design and planning. V.O., H.L., J.K., I.S., R.T., M.H. and C.N.-H. interpreted data and wrote the manuscript. M.H., C.N.-H., R.T. are shared last authors. 


\section{Declaration of interest}

584 The authors have nothing to declare. 


\section{$\underline{\text { References }}$}

5861 Krammer, F. SARS-CoV-2 vaccines in development. Nature 586, 516-527, doi:10.1038/s41586$587 \quad$ 020-2798-3 (2020).

5882 Baden, L. R. et al. Efficacy and Safety of the mRNA-1273 SARS-CoV-2 Vaccine. New England $589 \quad$ Journal of Medicine 384, 403-416, doi:10.1056/NEJMoa2035389 (2020).

5903 Polack, F. P. et al. Safety and Efficacy of the BNT162b2 mRNA Covid-19 Vaccine. New England 591 Journal of Medicine 383, 2603-2615, doi:10.1056/NEJMoa2034577 (2020).

5924 Sahin, U. et al. COVID-19 vaccine BNT162b1 elicits human antibody and TH1 T cell responses. 593 Nature 586, 594-599, doi:10.1038/s41586-020-2814-7 (2020).

5945 Sahin, U. et al. BNT162b2 induces SARS-CoV-2-neutralising antibodies and T cells in humans. 595 medRxiv, 2020.2012.2009.20245175, doi:10.1101/2020.12.09.20245175 (2020).

5966 Skelly, D. T. et al. Vaccine-induced immunity provides more robust heterotypic immunity than 597 natural infection to emerging SARS-CoV-2 variants of concern. Research Square, 598 doi:10.21203/rs.3.rs-226857/v1 (2021).

5997 Shirin, K. et al. Early T cell and binding antibody responses are associated with COVID-19 RNA 600 vaccine efficacy onset. Med, doi:https://doi.org/10.1016/j.medj.2021.04.003 (2021).

6018 Painter, M. M. et al. Rapid induction of antigen-specific CD $4<$ sup $>+</$ sup $>T$ cells guides 602 coordinated humoral and cellular immune responses to SARS-CoV-2 mRNA vaccination.

603 bioRxiv, 2021.2004.2021.440862, doi:10.1101/2021.04.21.440862 (2021).

6049 Widge, A. T. et al. Durability of Responses after SARS-CoV-2 mRNA-1273 Vaccination. New 605 England Journal of Medicine 384, 80-82, doi:10.1056/NEJMc2032195 (2020).

60610 Collier, D. A. et al. Sensitivity of SARS-CoV-2 B.1.1.7 to mRNA vaccine-elicited antibodies. $607 \quad$ Nature, doi:10.1038/s41586-021-03412-7 (2021).

60811 Wang, Z. et al. mRNA vaccine-elicited antibodies to SARS-CoV-2 and circulating variants. 609 Nature 592, 616-622, doi:10.1038/s41586-021-03324-6 (2021). 
61012 Goel, R. R. et al. Distinct antibody and memory B cell responses in SARS-CoV-2 naïve and 611 recovered individuals following mRNA vaccination. Science Immunology 6, eabi6950, 612 doi:10.1126/sciimmunol.abi6950 (2021).

61313 Joshi, N. S. et al. Inflammation directs memory precursor and short-lived effector CD8(+) T cell 614 fates via the graded expression of T-bet transcription factor. Immunity 27, 281-295, 615 doi:10.1016/j.immuni.2007.07.010 (2007).

61614 Boudousquié, C. et al. Differences in the Transduction of Canonical Wnt Signals Demarcate 617 Effector and Memory CD8 T Cells with Distinct Recall Proliferation Capacity. The Journal of 618 Immunology 193, 2784-2791, doi:10.4049/jimmunol.1400465 (2014).

61915 Ellebedy, A. et al. SARS-CoV-2 mRNA vaccines induce a robust germinal centre reaction in 620 humans. Research Square, doi:10.21203/rs.3.rs-310773/v1 (2021).

62116 Ellebedy, A. H. et al. Defining antigen-specific plasmablast and memory B cell subsets in human 622 blood after viral infection or vaccination. Nat Immunol 17, 1226-1234, doi:10.1038/ni.3533 $623 \quad$ (2016).

62417 Bange, E. M. et al. CD8 T cells compensate for impaired humoral immunity in COVID-19 625 patients with hematologic cancer. Res Sq, doi:10.21203/rs.3.rs-162289/v1 (2021).

$626 \quad 18 \quad$ Kalimuddin, S. et al. Early T cell and binding antibody responses are associated with Covid-19 627 RNA vaccine efficacy onset. Med (New York, N.Y.), doi:10.1016/j.medj.2021.04.003 (2021).

62819 Lederer, K. et al. SARS-CoV-2 mRNA Vaccines Foster Potent Antigen-Specific Germinal Center 629 Responses Associated with Neutralizing Antibody Generation. Immunity 53, 1281-1295.e1285, 630 doi:10.1016/j.immuni.2020.11.009 (2020).

63120 Akondy, R. S. et al. Origin and differentiation of human memory CD8 T cells after vaccination. 632 Nature 552, 362-367, doi:10.1038/nature24633 (2017).

63321 Kaech, S. M. \& Ahmed, R. Memory CD8+ T cell differentiation: initial antigen encounter triggers 634 a developmental program in naïve cells. Nature Immunology 2, 415-422, doi:10.1038/87720 $635 \quad$ (2001). 
63622 Romero, P. et al. Four functionally distinct populations of human effector-memory CD8+ T

637 lymphocytes. Journal of immunology (Baltimore, Md. : 1950) 178, 4112-4119, 638 doi:10.4049/jimmunol.178.7.4112 (2007).

63923 Schulien, I. et al. Characterization of pre-existing and induced SARS-CoV-2-specific CD8+ T cells. $640 \quad$ Nature Medicine 27, 78-85, doi:10.1038/s41591-020-01143-2 (2021).

64124 Sievers, F. et al. Fast, scalable generation of high-quality protein multiple sequence alignments 642 using Clustal Omega. Molecular systems biology 7, 539, doi:10.1038/msb.2011.75 (2011).

64325 Wieland, D. et al. TCF1(+) hepatitis C virus-specific CD8(+) T cells are maintained after cessation 644 of chronic antigen stimulation. Nature communications 8, 15050, doi:10.1038/ncomms15050 645 (2017).

64626 Alanio, C., Lemaitre, F., Law, H. K., Hasan, M. \& Albert, M. L. Enumeration of human antigen647 specific naive CD8+ T cells reveals conserved precursor frequencies. Blood 115, 3718-3725, 648 doi:10.1182/blood-2009-10-251124 (2010).

649 


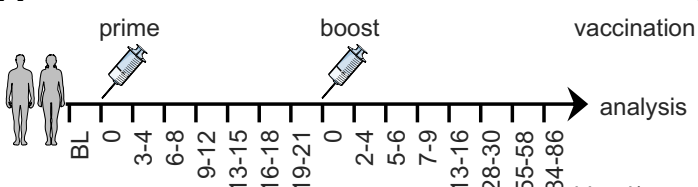

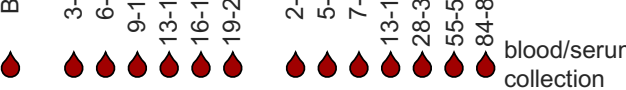

dpp

$\mathrm{dpb}$

C Spike-specific CD8 ${ }^{+} \mathrm{T}$ cells calc. ex vivo frequency

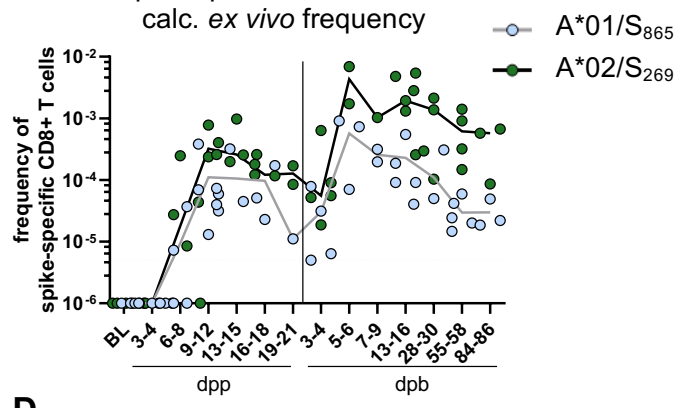

CD38+

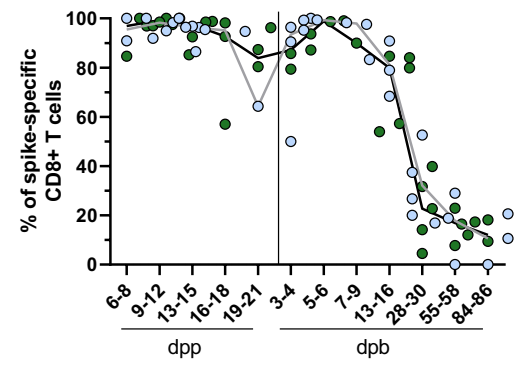

E
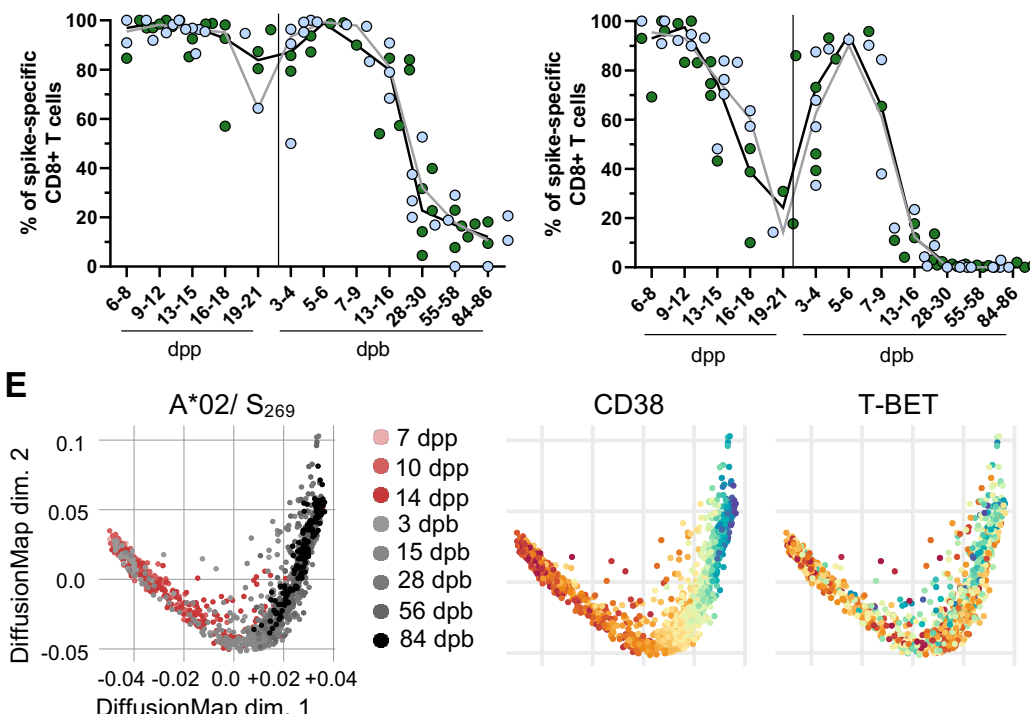

CD38

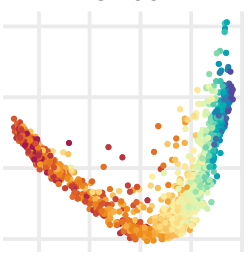

T-BET

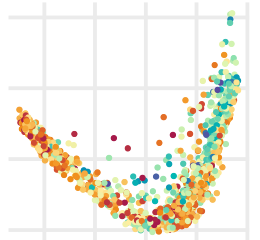

ex vivo spike-specific CD8 ${ }^{+} \mathrm{T}$ cells (enriched)

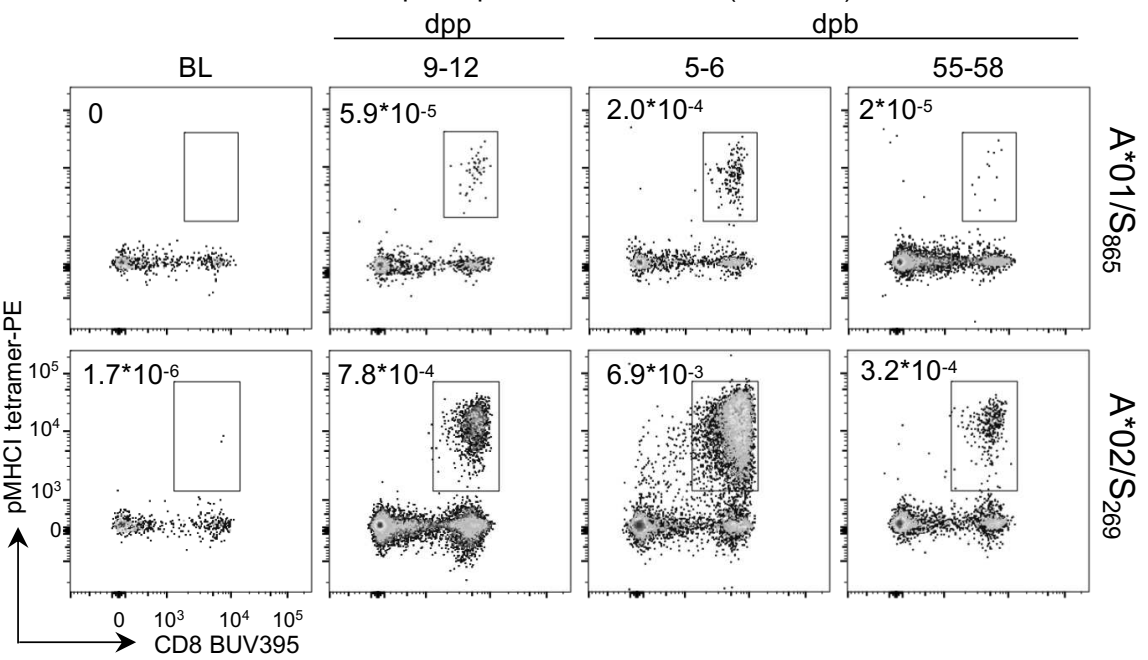

T-BEThi
$\mathbf{F}$

CD127

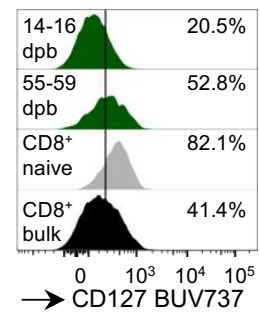

Spike-specific CD127+ CD8+ T cells calc. ex vivo frequencies

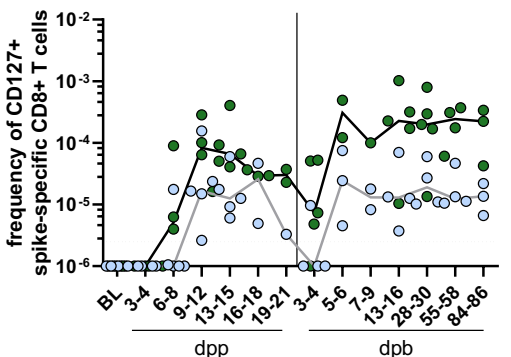

G TCF-1

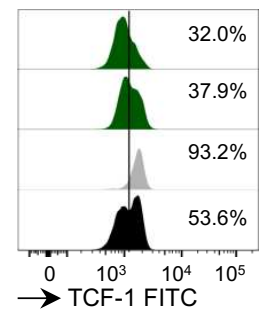

Spike-specific TCF-1+ CD8+ T cells

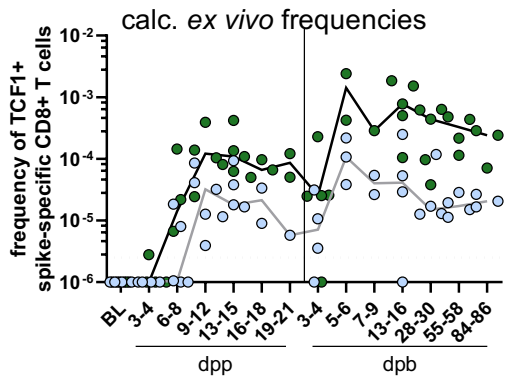

Figure 1: Bnt162b2 vaccine-elicited epitope-specific CD8+ T cells: early induction, vigorous Teff response and robust establishment of memory precursors

(A) Timeline showing blood and serum collection before and after prime and boost vaccination. (B) Dot plots showing $A^{*} 01 / S_{865-}$ and $A^{*} 02 / S_{269}$-specific $C D 8+T$ cells ex vivo after $\mathrm{pMHCl}$ tetramer-based enrichment at $\mathrm{BL}$, before and after boost vaccination. (C) The calculated ex vivo frequency is indicated at $B L$, dpp and dpb for $A^{*} 01 / S_{865^{-}}$and $A^{*} 02 / S_{269}$-specific CD8+ T cells. (D) $\%$ of CD38, Ki-67 and T-BET ${ }^{\text {hi }}$ expressing $A^{*} 01 / S_{865^{-}}$and $A^{*} 02 / S_{269}$-specific non-naïve CD8+ $T$ cells. (E) Diffusion map showing flow cytometry data for $A^{*} 02 / S_{269}$-specific CD8+ T cells in relation to dpp (shades of red) and dpb (shades of grey) in one individual. CD38, T-BET, TOX and BCL-2 expression levels are plotted on the diffusion map (color-code: blue, low expression; red, high expression). (F-G) Exemplary histograms depicting the expression levels of CD127 and TCF-1 in $A^{*} 02 / S_{269^{-}}\left(g_{r e e n}\right)$ specific, naïve (grey) and bulk (black) CD8+ T cells 14-16 and 55-59 dpb. Calculated ex vivo frequencies of non-naïve spike-specific CD8+ T cells expressing CD127 or TCF-1 for $A^{*} 01 / S_{865^{-}}$and $A^{*} 02 / S_{269-}$ specific CD8+ T cells. BL: baseline; dpp: days post prime; dpb: days post boost. 
A

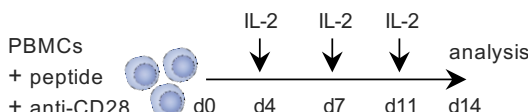

$\begin{array}{llllll}+ \text { anti-CD28 d0 } & \text { d4 } & \text { d7 } & \text { d11 } & \text { d14 }\end{array}$

B

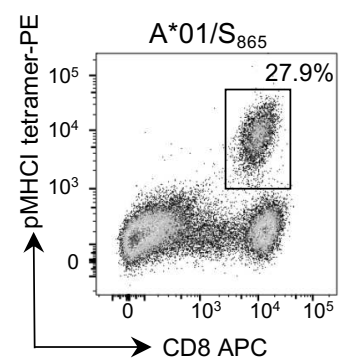

E

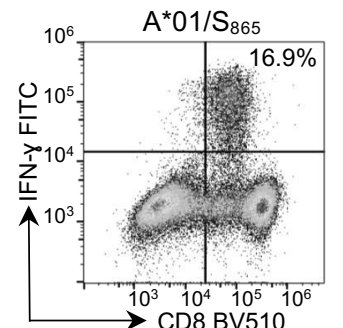

IFN- $\gamma^{+}$
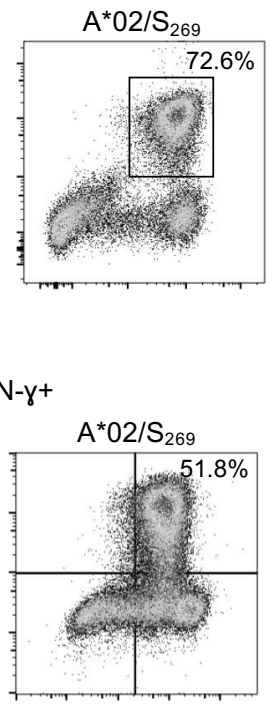

IFN- $\gamma+$ / tet

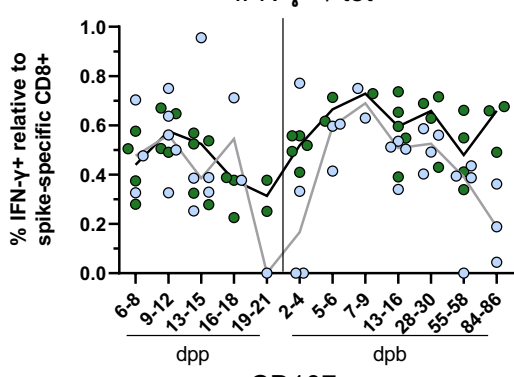

CD107a+

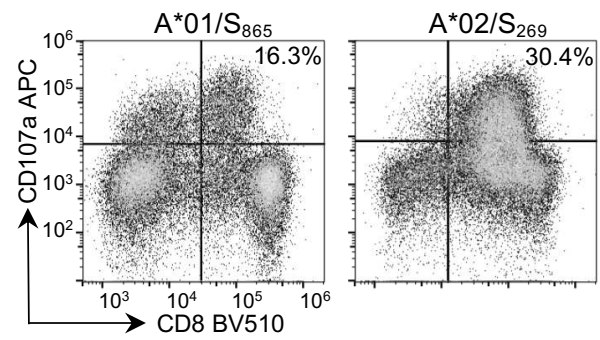

C

Frequency after in vitro expansion

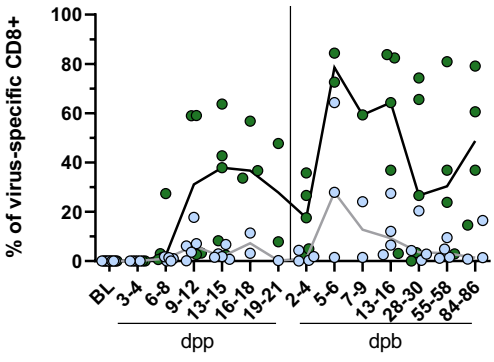

$\mathbf{F}$

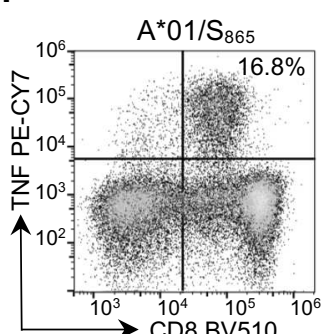

TNF+

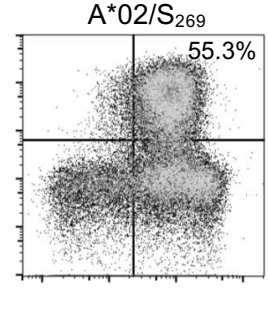

$\mathrm{TNF}+/$ tet
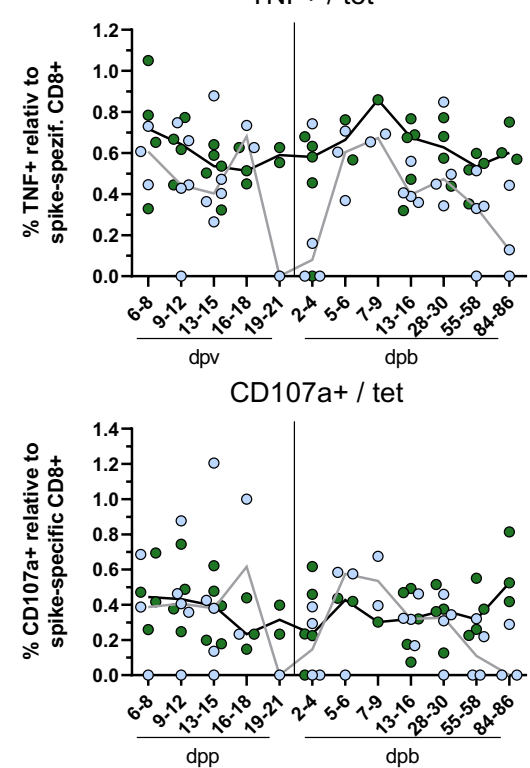

D

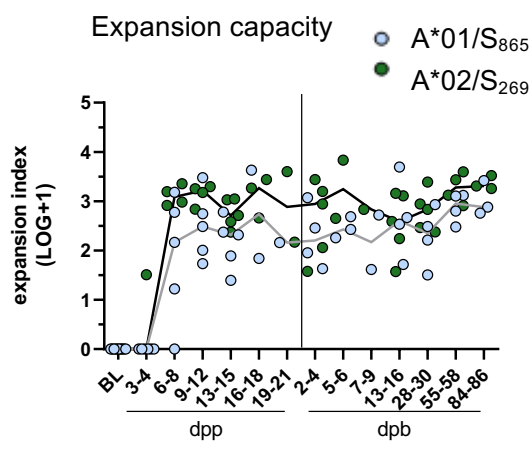

G

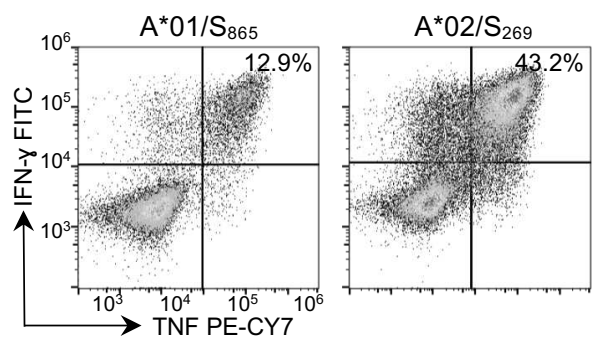

IFN- $\gamma+$ TNF $+/$ tet

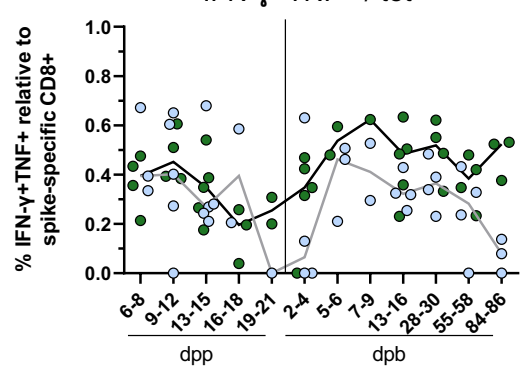

Figure 2: Proper and conserved functional capacities of circulating spike-specific CD8+ T cells following prime and boost vaccination

(A) Workflow depicting peptide-specific in vitro expansion of CD8+ T cells. (B) Dot plots showing $A^{*} 01 / S_{865-}$ and $A^{*} 02 / S_{269}$-specific CD8+ T cells after in vitro expansion. (C, D) Frequency and expansion capacity of the respective spike-specific CD8+ $T$ cells after in vitro expansion. (E-H) Dot plots showing IFN-ץ- and TNF-, IFN-y /TNF- and CD107a-expressing CD8+ T cells after in vitro expansion. \% of $C D 8+T$ cells producing the respective cytokines in relation to the frequency of $A^{*} 01 / S_{865}$ and $A^{*} 02 / S_{269}$-specific CD8+ T cells was determined. Dot plot show samples 5-6 dpb. BL: baseline; dpp: days post prime; dpb: days post boost. 


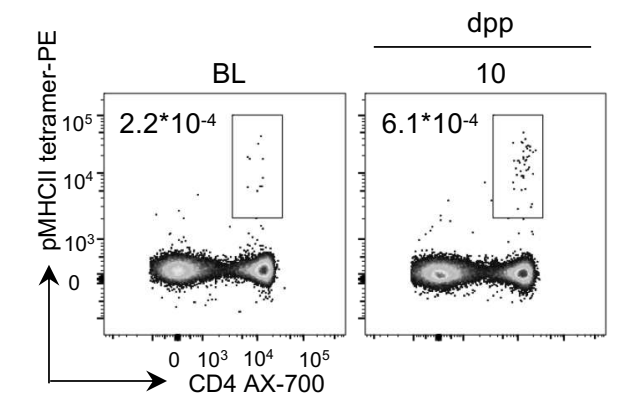

B

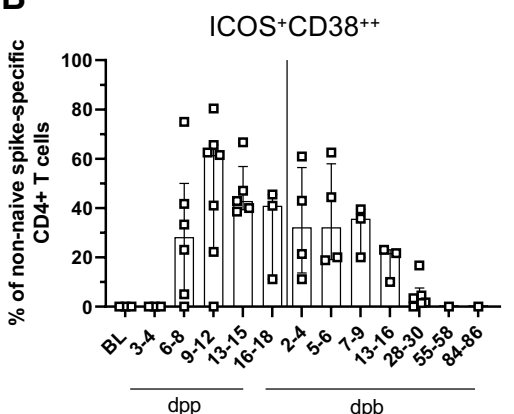

D

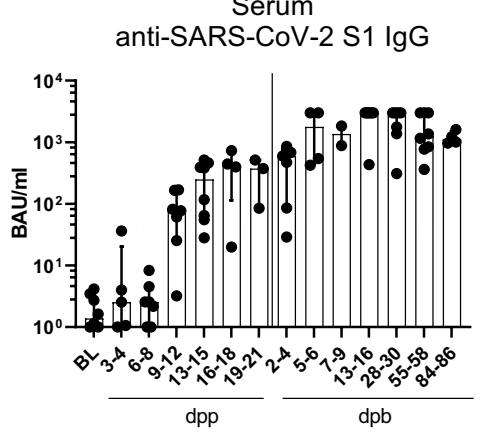

G

Spike-specific B cells

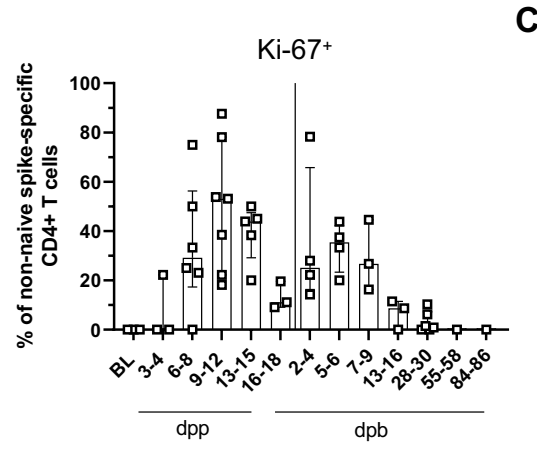

E

Neutralization of

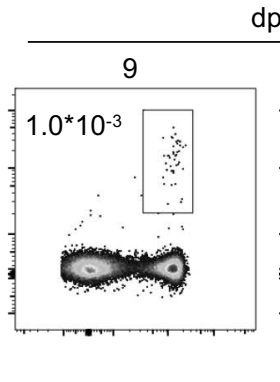

$\mathrm{dpb}$

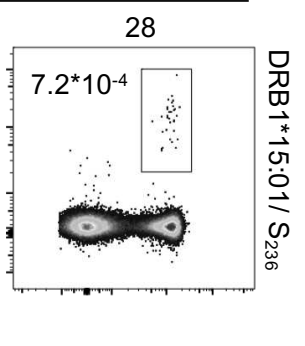

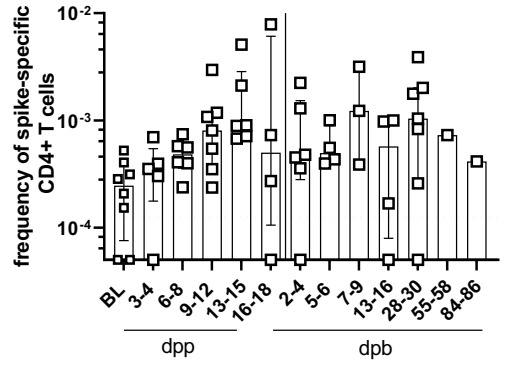

Ki-67+
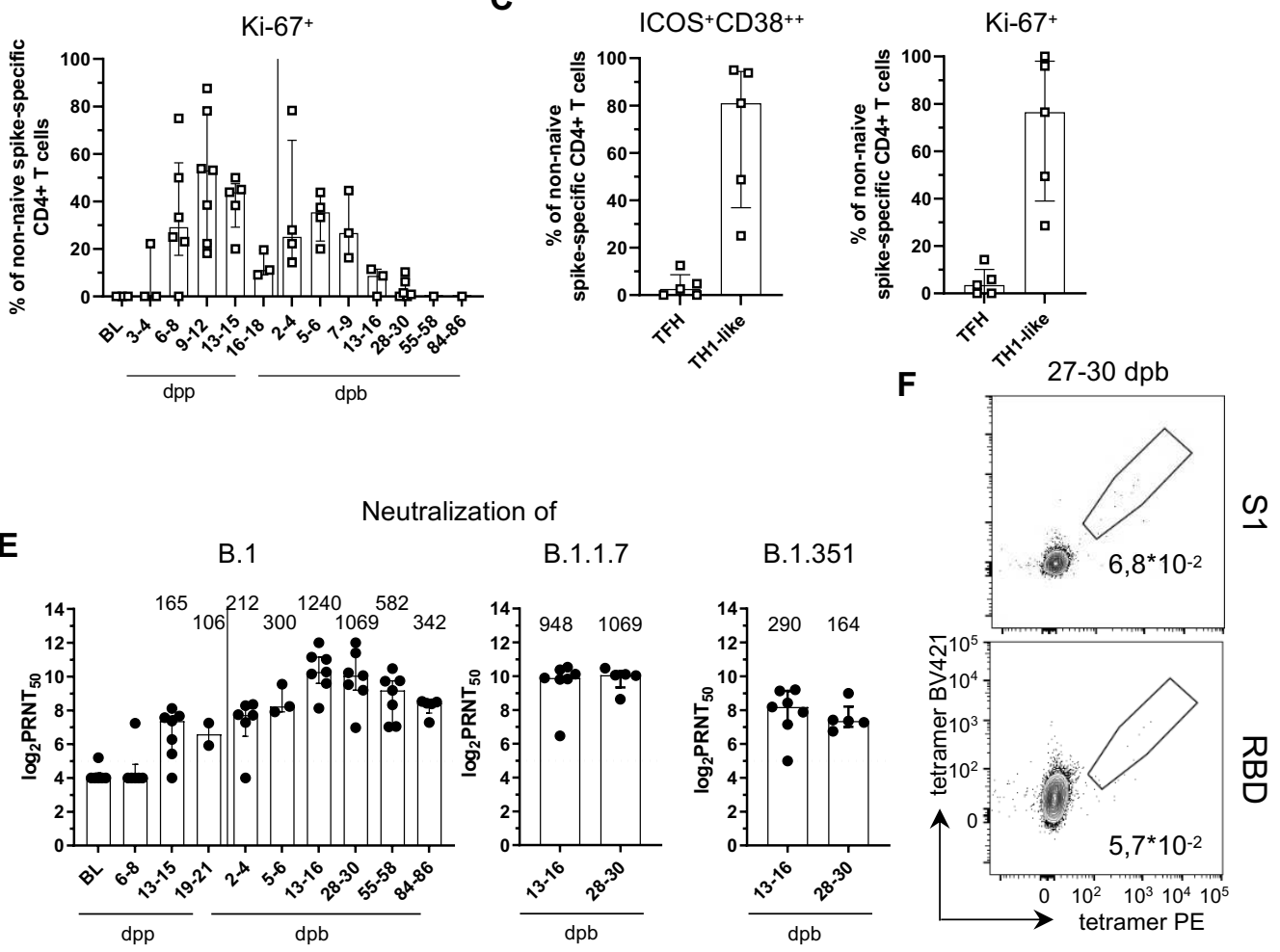

B.1.351

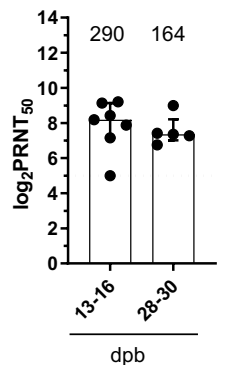

$\mathrm{dpb}$

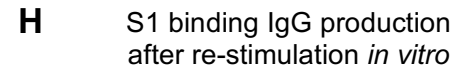

I

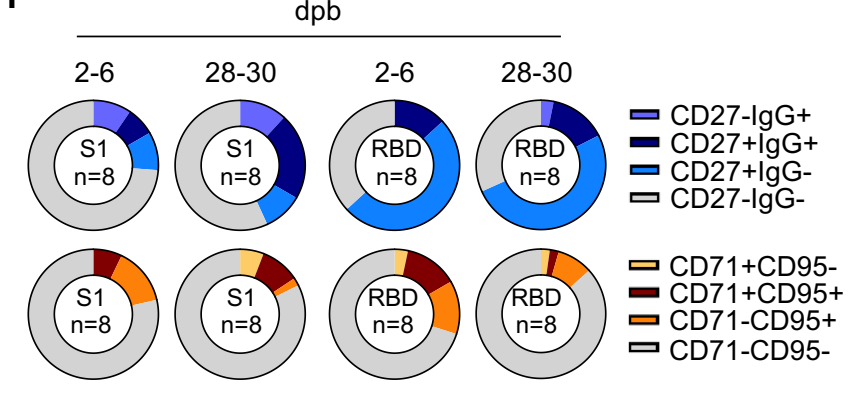

Figure 3: Delayed appearance of circulating spike-specific CD4+ T cells, B cells and antibodies

(A) Dot plots showing DRB1*15:01/ $\mathrm{S}_{236}$-specific CD4+ T cells ex vivo after pMHCll tetramer-based enrichment at BL and after prime/ boost. The bar graph shows the calculated ex vivo frequency of DRB1*15:01/S 236 -specific CD4+ T cells. (B) ICOS+CD38++ and Ki-67 expression within non-naïve, DRB1*15:01/S 236 -specific CD4+ T cells (C) ICOS+CD38++ and Ki-67-expressing non-naïve DRB1*15:01/S 236 -specific CD4+ T cells on 9-2 dpp within the population of TFH (CXCR5+PD-1+) and TH1 (CXCR5-CXCR3+) cells. (D) Anti-SARS-CoV-2 Spike IgG at BL and after prime and boost vaccination $(<35,2 \mathrm{BAU} / \mathrm{ml}$ : negative, $\geq 35,2 \mathrm{BAU} / \mathrm{ml}$ : positive). (E) Antibody neutralization activity is depicted as PRNT 50 at BL, dpp and dpb vaccination for the SARS-CoV-2 ancestral variant B.1 and the VOC B.1.1.7 and B.1.351 at 13-16 and 28-30 dpb when the highest neutralization titer was detected with the ancestral B.1 variant. Numbers indicate median value (F) Dot plots showing double tetramer positive B cells for S1 and RBD epitope. (G) \% of spike-specific B cells depicted at BL, dpp and dpb as well as in natural infection for S1 and RBD epitope. (H) PBMC from BL, dpp and dpb as well as samples after natural infection were stimulated in vitro for nine days with CpG and IL-2. Secreted anti-SARS-CoV-2 Spike IgG was detected in the supernatant by ELISA. (I) Donut plots representing co-expression of CD27/lgG (upper) and CD71/CD95 (lower) in concatenated analysis of S1-specific B cells populations ( $\mathrm{N}$ indicated the number of individual files concatenated) after boost vaccination. BL: baseline, dpp: days post prime, dpb: days post boost; TFH follicular helper T cells; TH1 T helper cells 1; PRNT50: plaque-reduction neutralization titer 50; BAU: antibody units; RU: arbitrary units. Bar charts show the median with IQR. 

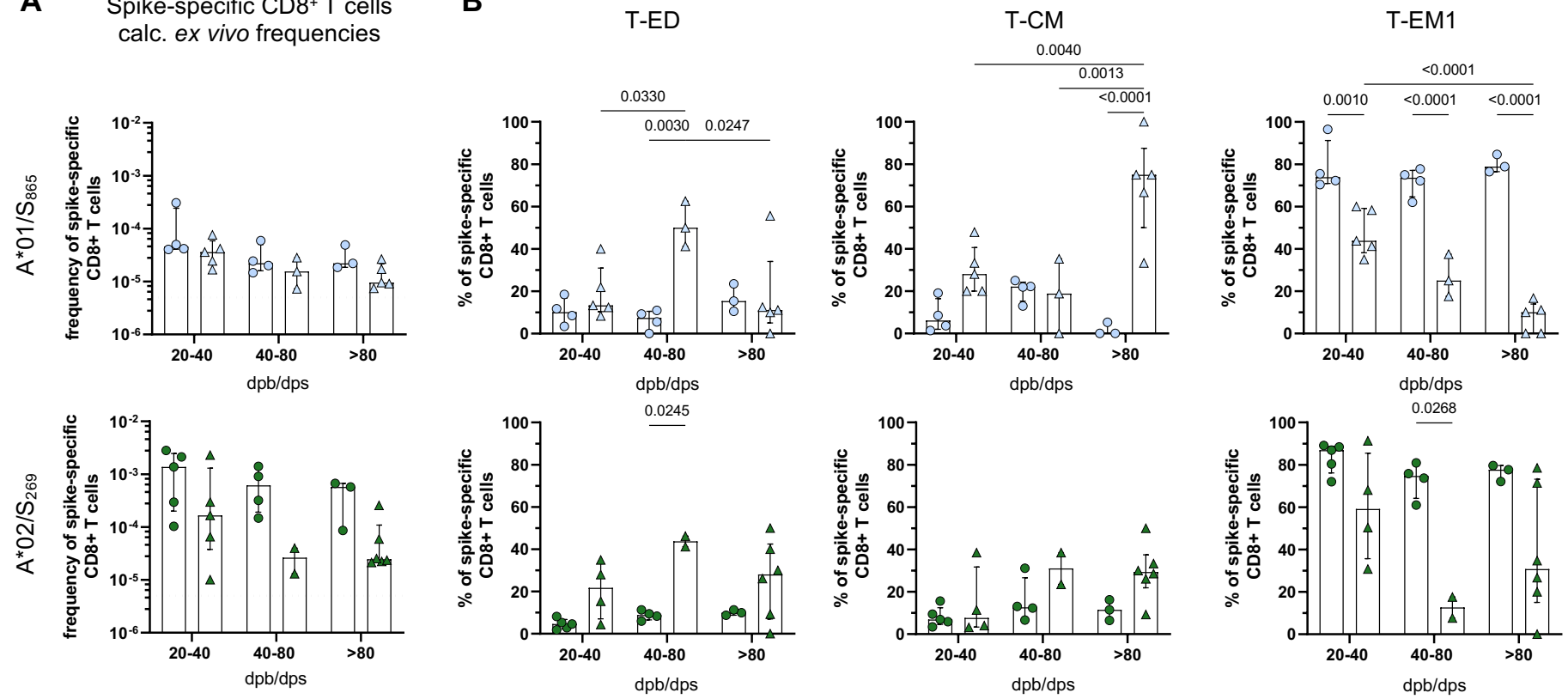

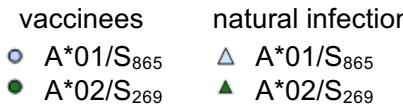

C

$$
\text { - } \mathrm{A}^{*} 02 / \mathrm{S}_{269} \Delta \mathrm{A}^{*} 02 / \mathrm{S}_{269}
$$
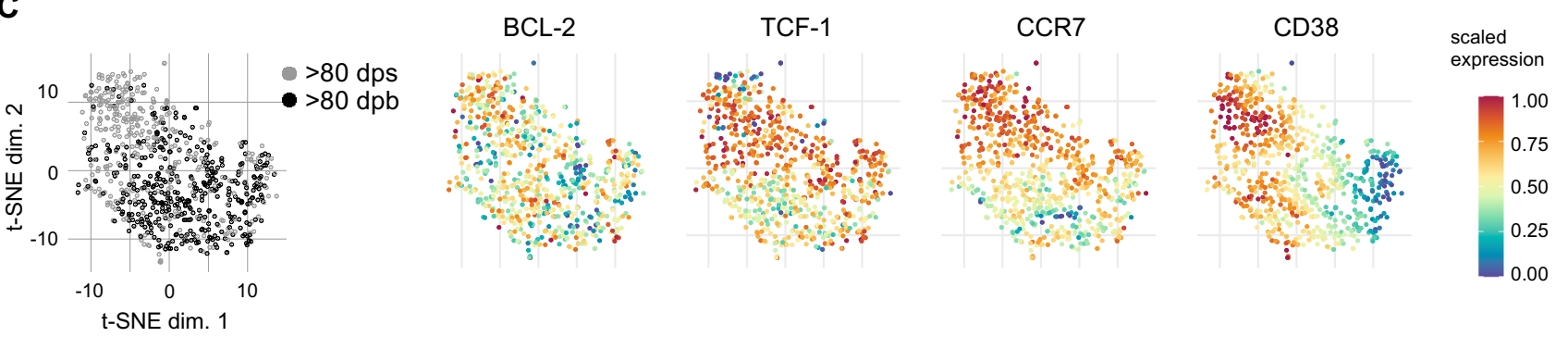

D
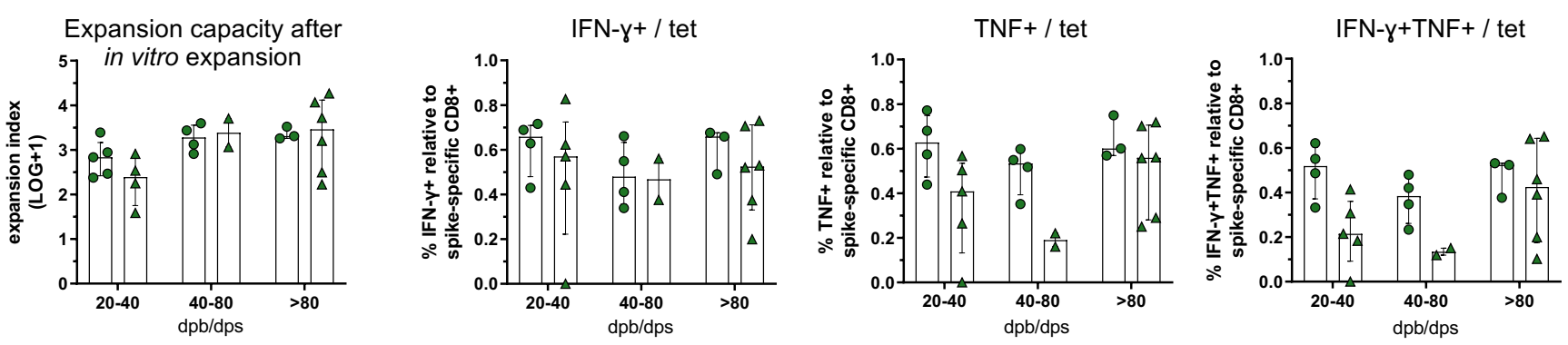

Figure 4: Differences of circulating spike-specific early memory CD8+ T cells after boost vaccination and natural infection (A) Calculated frequency of $A^{*} 01 / S_{865^{-}}$and $A^{*} 02 / S_{269}$-specific CD8+ $T$ cells after boost vaccination or natural SARS-CoV-2 infection ex vivo after $\mathrm{pMHCl}$ tetramer-based enrichment. (B) Distribution of CD8+ T cell memory subsets, TED, TCM and TEM1,

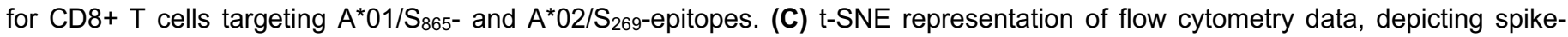
specific CD8+ T cells more than $80 \mathrm{dpb}$ and dps of natural infection (grey: natural infection, black: vaccines). BCL-2, TCF-1, CCR7 and CD38 expression levels are plotted on the t-SNE (color-code: blue, low expression; red, high expression). (D) Frequency of the respective spike-specific CD8+ T cells after in vitro expansion. \% of IFN- $\gamma-$ and TNF-producing as well as IFN- $\gamma$ and TNF coproducing CD8+ T cells in relation to the frequency of $A^{*} 02 / S_{269}$-specific CD8+ T cells after in vitro expansion. Bar charts show the median with IQR. $A^{*} 01 / S_{865} n=5$ vaccinees longitudinally and $n=10$ naturally infected patients cross-sectional/longitudinally; $A^{*} 02 / S_{269} n=5$ vaccinees longitudinally and $n=12$ naturally infected patients cross-sectional. 2way ANOVA including Tukey's multiple comparisons test were performed. dpb: days post boost; dps: days post symptoms; TED: early differentiated, TCM: central memory T cells and TEM1: effector memory T cells 1; t-SNE: t-distributed stochastic neighbor embedding. 

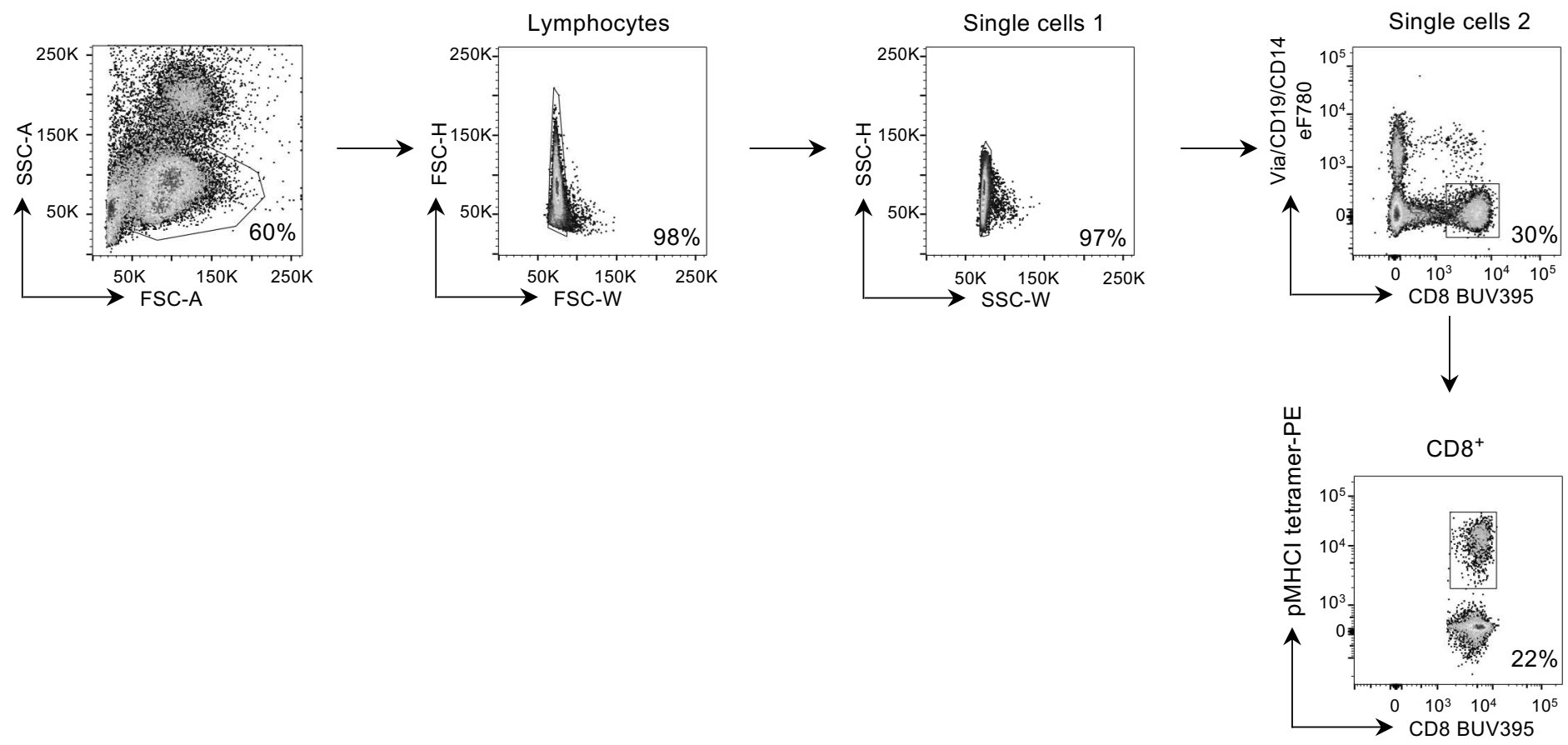

\section{Extended Data Figure 1:}

Gating strategy of flow cytometry data. $A^{*} 01 / S_{865^{-}}$and $A^{*} 02 / S_{269}$-specific $C D 8+T$ cells were identified via $\mathrm{pMHCl}$ tetramer-based enrichment used for further analyses. 
A
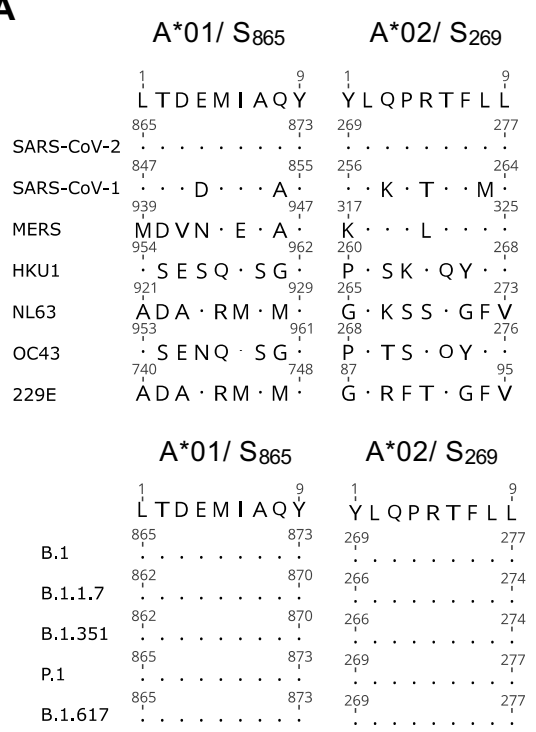

C
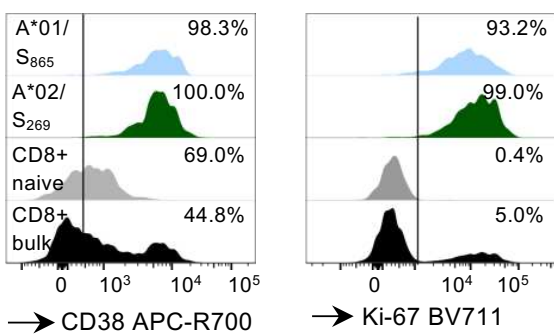

D

GRZB+
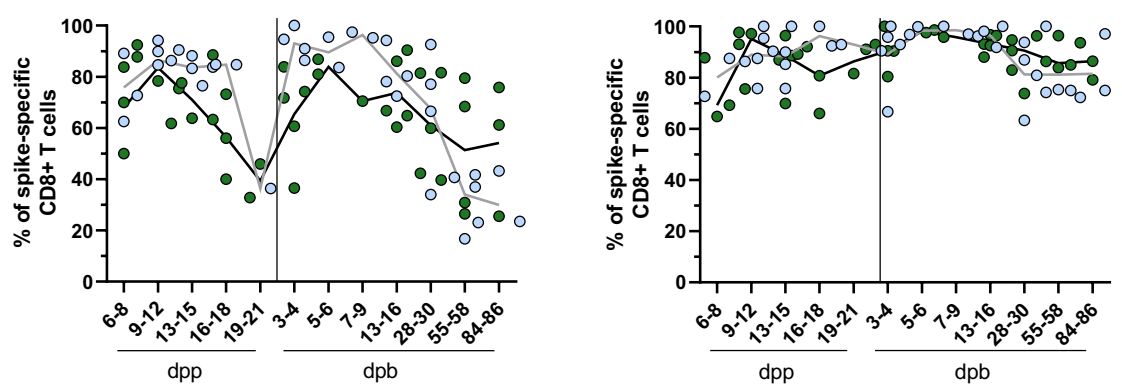

E
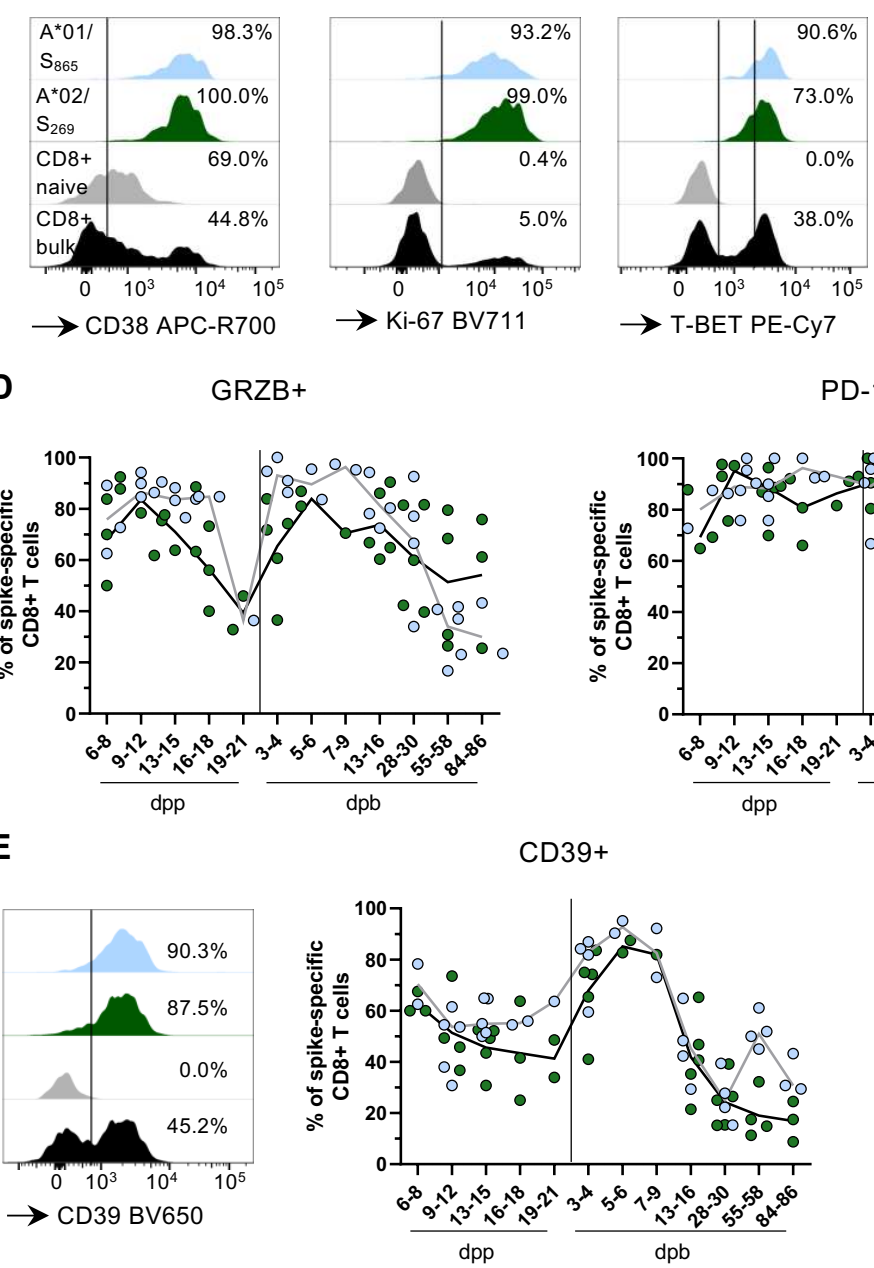

ex vivo spike-specific CD8+ T cells (without enrichment)
B

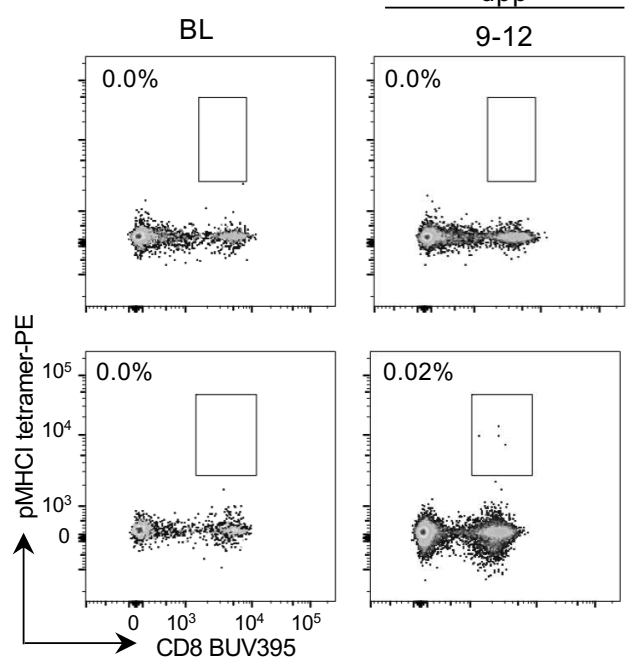

$\mathrm{dpb}$
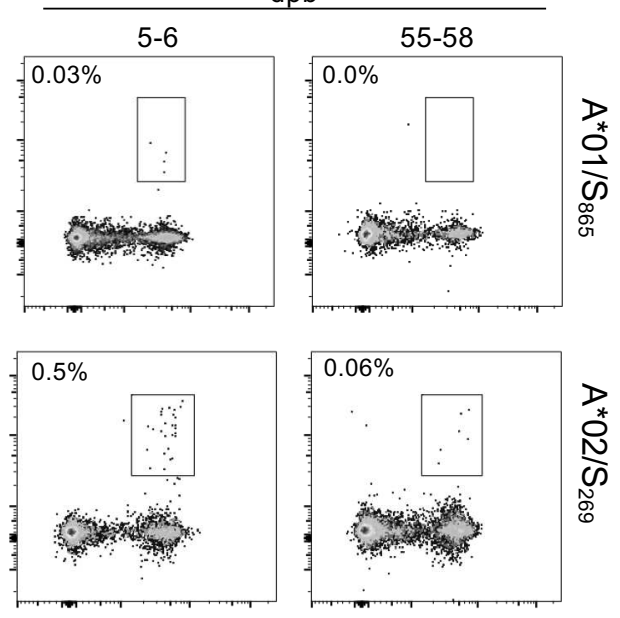
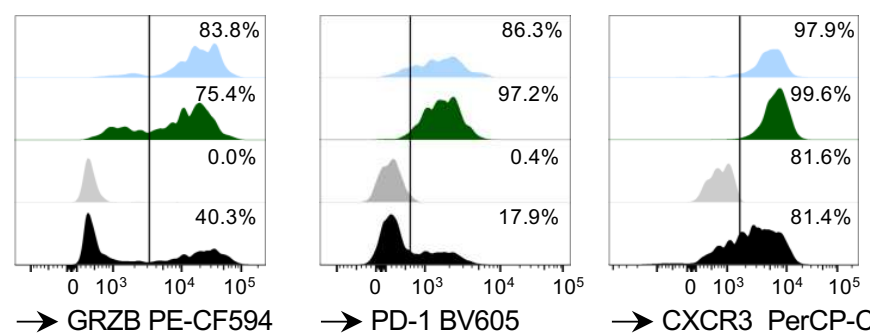

$\rightarrow$ CXCR3 PerCP-Cy5.5

CXCR3+
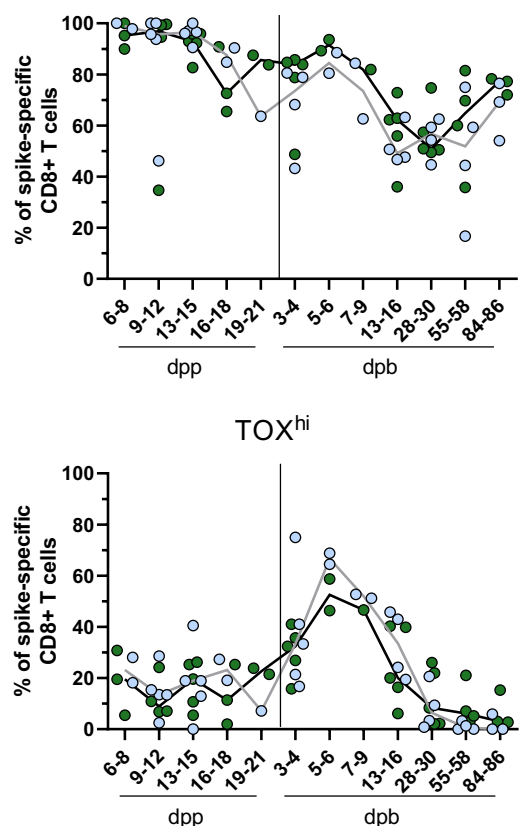

Extended Data Figure 2:

(A) Comparison of epitope sequences with amino acid sequences of SARS-CoV-1/2, MERS and common cold coronaviruses amino acid sequences (upper panel) and with circulating SARS-CoV-2 variants of concern (VOC) (lower panel), respectively for $A^{*} 01 / S_{865}$ and $A^{*} 02 / S_{269} C D 8+T$ cell epitopes. (B) Dot plots showing $A^{*} 01 / S_{865}$ - and $A^{*} 02 / S_{269}$-specific $C D 8+T$ cells ex vivo without pMHCl tetramer-based enrichment. (C) Exemplary histograms (13-15 dpp) depicting the expression levels of CD38, Ki-67, T-BET, GRZB, PD-1 and CXCR3 in $A^{*} 01 / S_{865^{-}}$(blue) and $A^{*} 02 / S_{269-}$ (green) specific, naïve (greay) an bulk (black) CD8+ $T$ cells. (D) \% of granzyme $B, P D-1$ and CXCR3 expressing $A^{*} 01 / S_{865^{-}}$and $A^{*} 02 / S_{269}$-specific non-naïve CD8+ T cells. (E) Exemplary histograms (5-6 dpb) depicting the expression levels of CD39 and TOX in $A^{*} 01 / S_{865-}$ (blue) and $A^{*} 02 / S_{269-}$ (green) specific, naïve (grey) an bulk (black) CD8+ T cells. \% expressing $A^{*} 01 / S_{865}$ and $A^{*} 02 / S_{269}$-specific non-naïve CD8+ $T$ cells is shown on the ride side. BL: baseline; dpp: days post prime; dpb: days post boost; GRZB: granzyme B. 
A
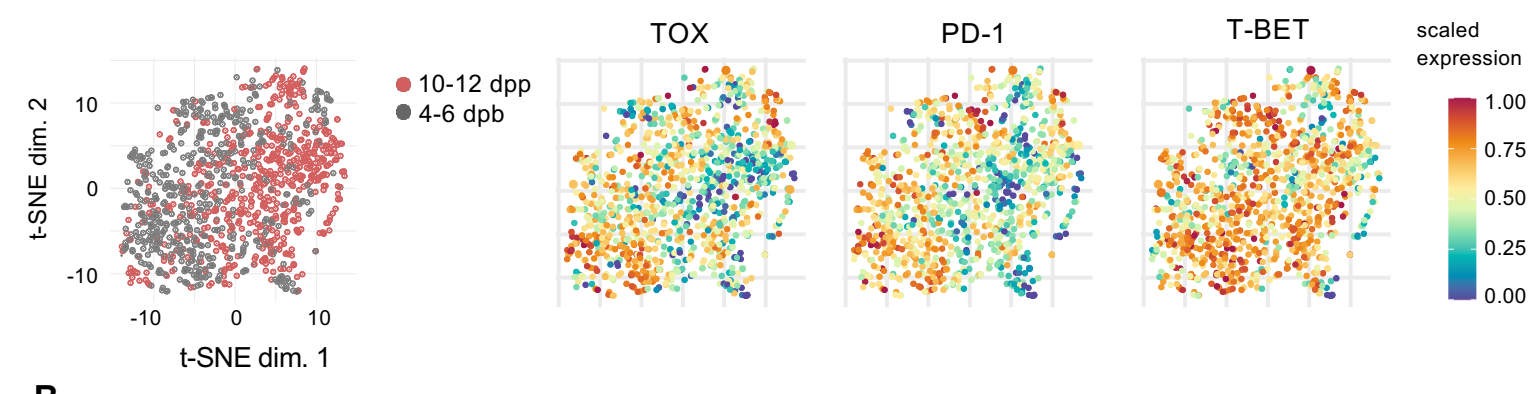

B
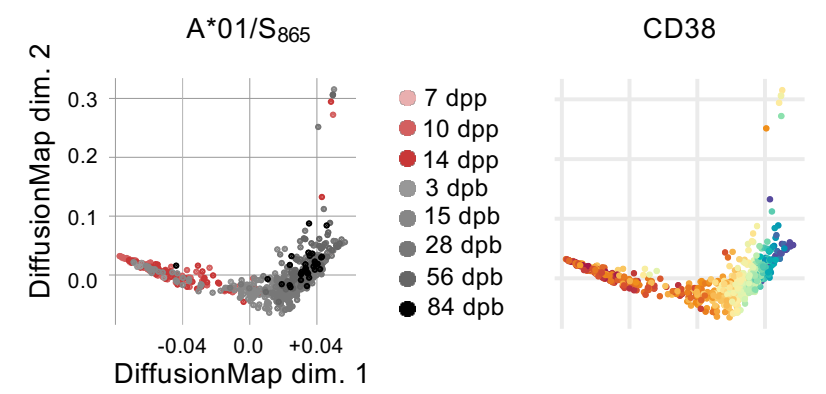

T-BET

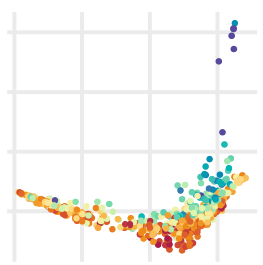

TOX

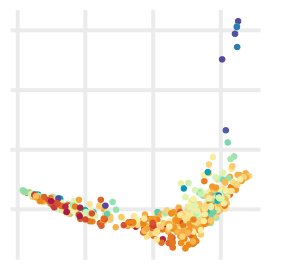

BCL-2

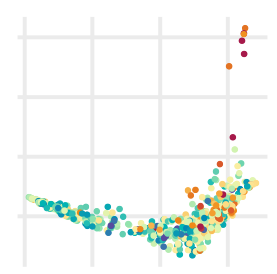

C

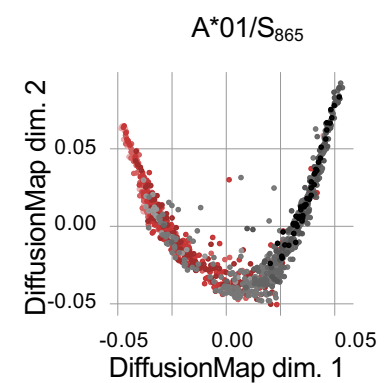

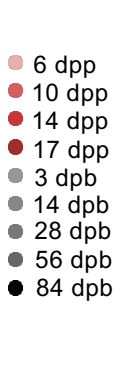

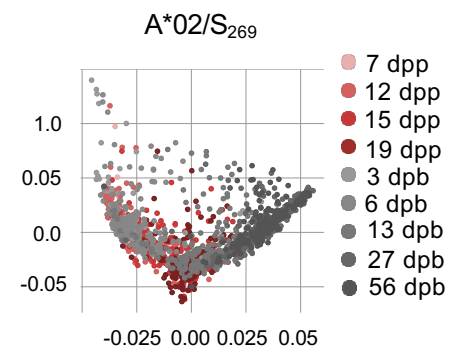

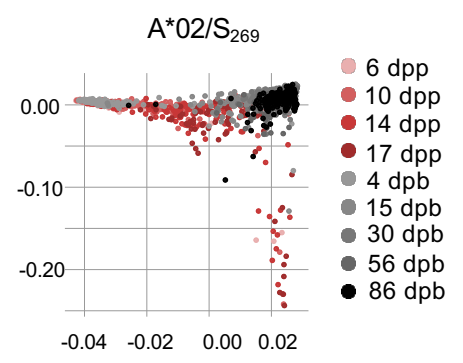

\section{Extended Data Figure 3:}

(A) t-SNE representation of flow cytometry data comparing spike-specific CD8+ T cells after prime and boost vaccination of 7 individuals after prime $\left(4 A^{*} 01 / S_{865}, 3 A^{*} 02 / S_{269}\right)$ and 6 individuals after boost $\left(3 A^{*} 01 / S_{865}, 3 A^{*} 02 / S_{269}\right)$. Expression levels of TOX, PD-1 and T-BET are indicated (color-code: blue, low expression; red, high expression). (B) Diffusion map showing flow cytometry data for $A^{*} 01 / S_{865}$-specific CD8+ T cells at dpp (shades of red) and dpb (shades of grey) with CD38, T-BET, TOX and BCL-2 expression levels plotted on the diffusion map (color-code: blue, low expression; red, high expression). (C) Diffusion map showing flow cytometry data for $A^{*} 01 / S_{865^{-}}$and $A^{*} 02 / S_{269}$-specific $C D 8+T$ cells at dpp (shades of red) and $\mathrm{dpb}$ (shades of grey) for two individuals each. dpp: days post prime; dpb: days post boost; t-SNE: t-distributed stochastic neighbor embedding. 
A

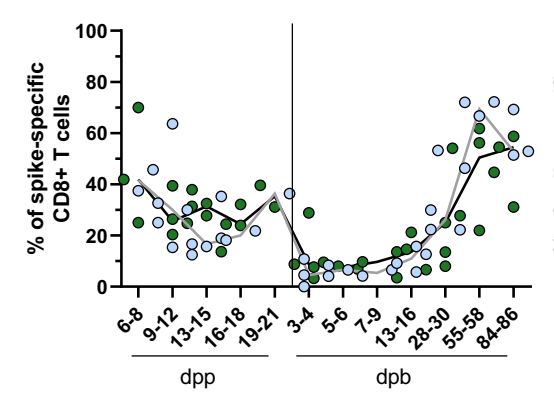

CD127+
B

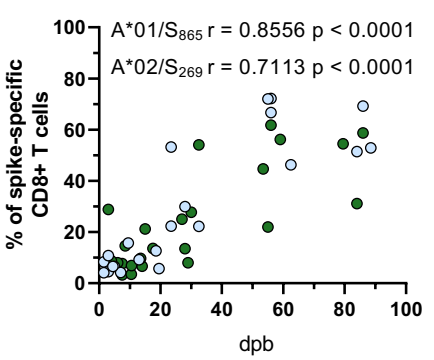

TCF-1+

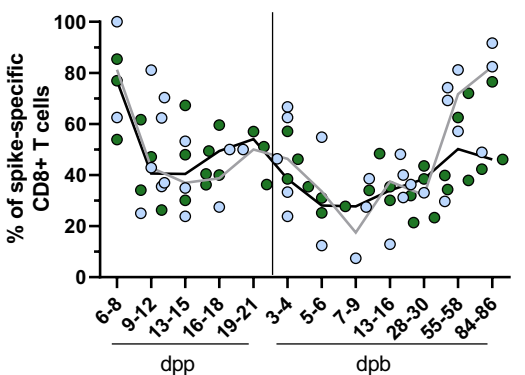

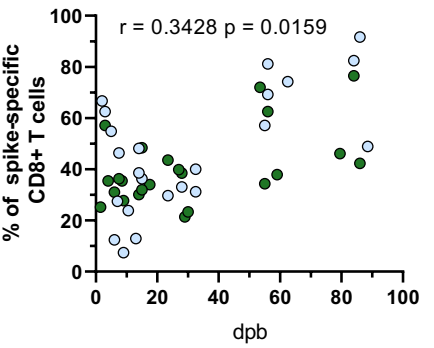

C

$\mathrm{A}^{*} 02 / \mathrm{S}_{269}$

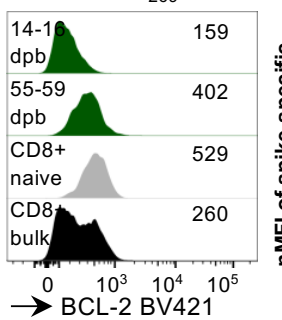

BCL-2
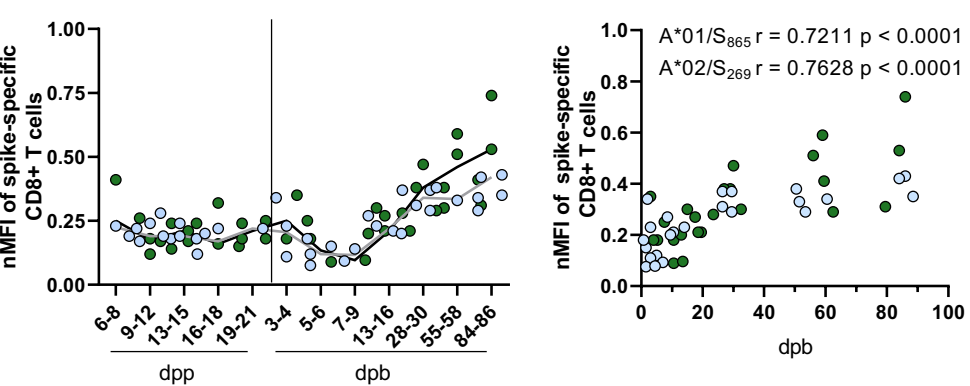

$\multimap A^{*} 01 / S_{865}$

$\rightarrow A^{*} 02 / S_{269}$

Extended Data Figure 4:

(A-B) \% of $A^{*} 01 / S_{865}$ and $A^{*} 02 / S_{269}$-specific non-naïve CD8+ T cells expressing CD127 and TCF-1 were determined. Correlation of indicated marker with $\mathrm{dpb}$ is depicted on the right. (C) Exemplary histograms depicting the expression levels of BCL-2 on $\mathrm{A}^{*} 02 / \mathrm{S}_{269}$ - (green) specific CD8+ T cells as well as on naïve CD8+ T cells (grey) and CD8+ bulk (black) 14-16 and 55-59 dpb. nMFI (normalized to naïve CD8+ $T$ cells) of $A^{*} 01 / S_{865^{-}}$and $A^{*} 02 / S_{269}$-specific non-naïve $C D 8+T$ cells expressing $B C L-2$ was determined. Correlation of nMFI BCL-2 with $d p b$ is depicted on the right. For (A) $A^{*} 01 / S_{865} n=23$ for $A^{*} 02 / S_{269} n=25$ in (B, C) $\left(A^{*} 01 / S_{865} n=24\right.$ for $\left.A^{*} 02 / S_{269} n=25\right)$ Spearman correlation was performed. BL: baseline; dpp: days post prime; dpb: days post boost; nMFI: normalized Median Fluorescent Intensity. 
A
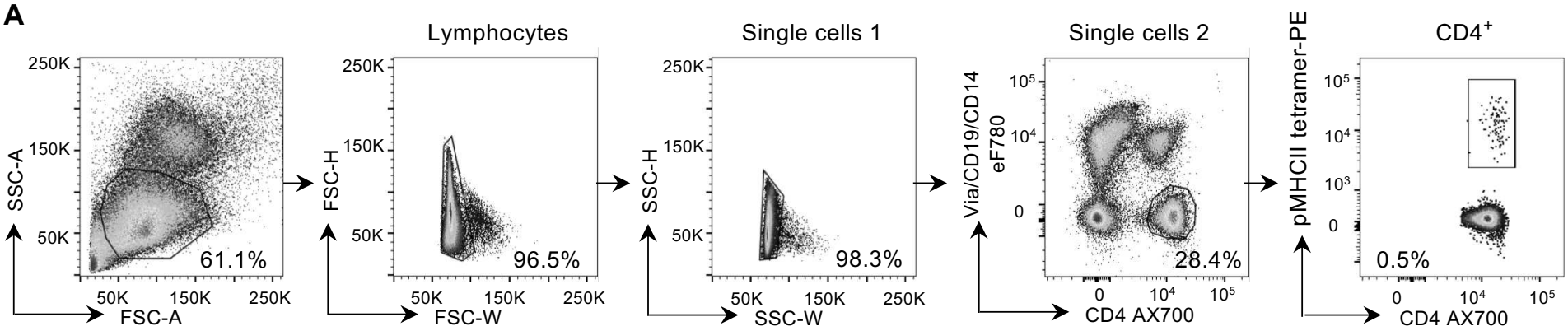

B

C

Historic controls (HC)
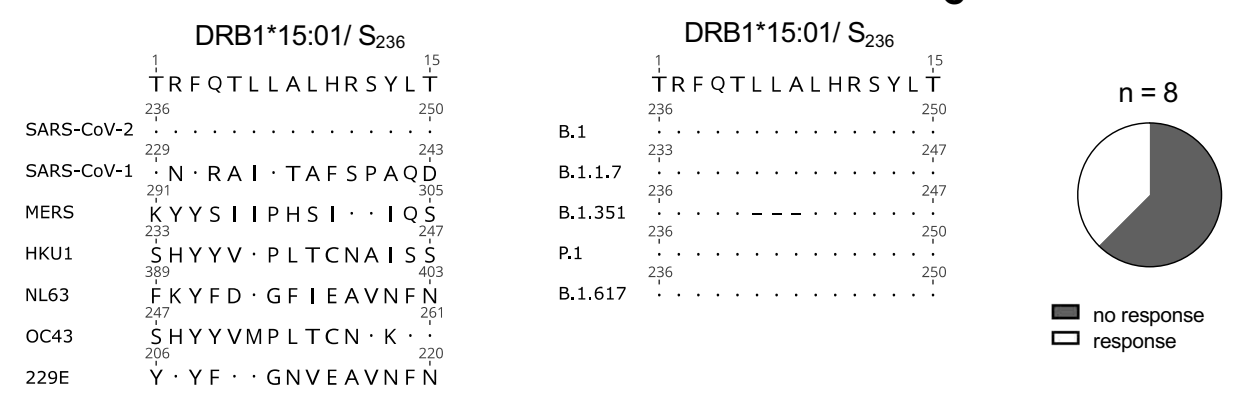

$\square$ no response
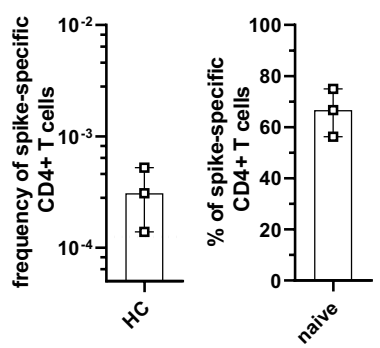

D

T-naive

$\mathbf{E}$

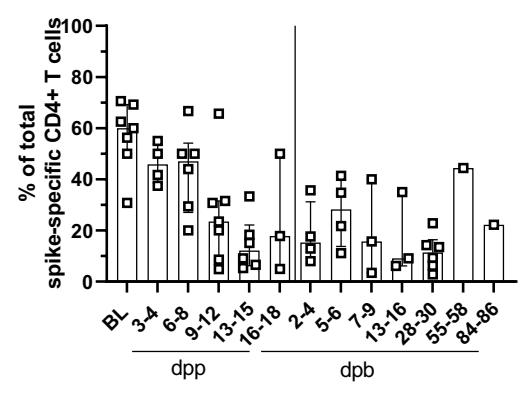

Non-naive CD4+

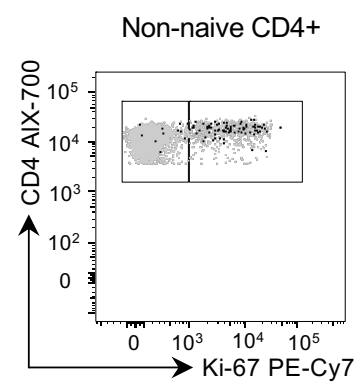

TH1-like: CXCR5-CXCR3+

Non-naive CD4+

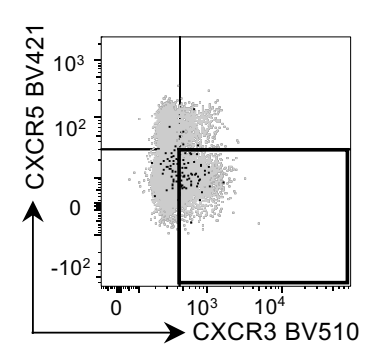

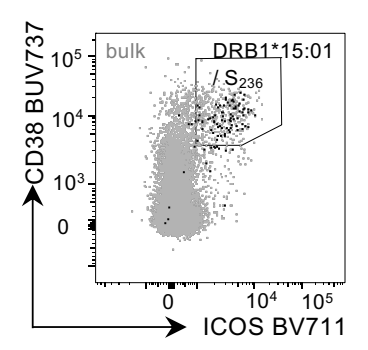

TFH: CXCR5+PD-1+

$\mathbf{F}$
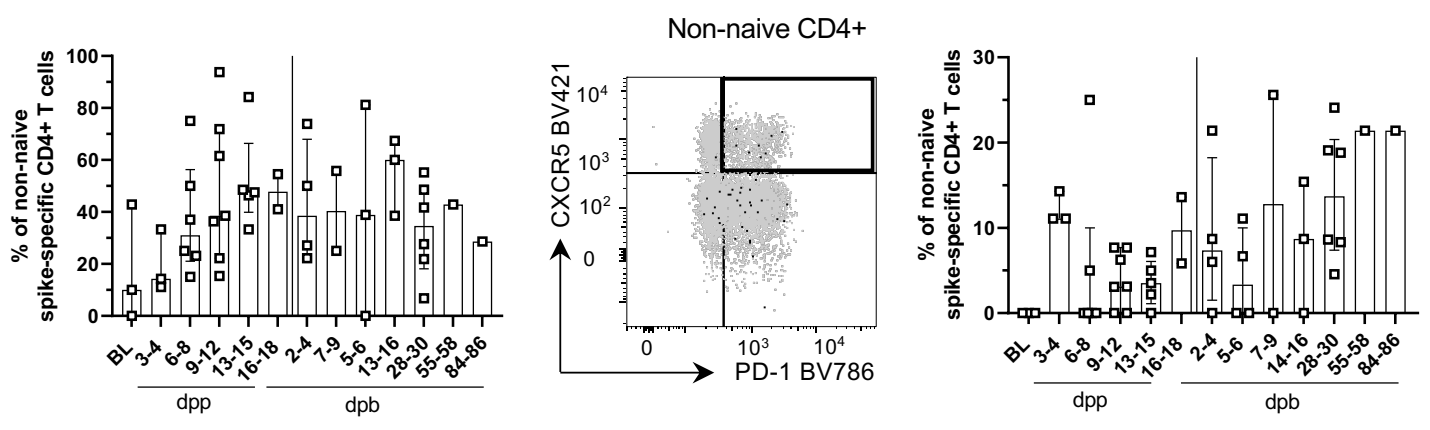

\section{Extended Data Figure 5:}

(A) Gating strategy of flow cytometry data. DRB1*15:01/S ${ }_{236}$-specific CD4+ T cells were identified via pMHCII tetramer-based enrichment used in further analyses. (B) Comparison of DRB1*15:01/S ${ }_{236}$ epitope sequence with amino acid sequences of SARSCoV-1/2, MERS and common cold coronaviruses (left) and of circulating SARS-CoV-2 variants of concern (VOC) (right). (C) Number of responses (left) and the calculated ex vivo frequencies (middle) of DRB1*15:01/S 236 -specific CD4+ T cells in historic controls (HC, $n=8)$. \% naïve within total DRB1*15:01/S 236 -specific CD4+ T cells in HC (right). (D) \% naïve of total DRB1*15:01/S $236-$ specific CD4+ T cells. (E) Representative dot plots of ICOS+CD38++ and Ki-67+ expression (grey: bulk, black: DRB1*15:01/S $236-$ specific CD4+ T cells). (F) \% CXCR5-CXCR3+ TH1 cells and of CXCR5+PD-1+ TFH within non-naïve DRB1*15:01/S $236-S p e c i f i c$ CD4+ T cells with representative dot plots (grey: bulk, black: DRB1*15:01/S 236 -specific CD4+ T cells). BL: baseline, dpp: days post prime, dpb: days post boost; TFH follicular helper T cells; TH1 T helper cells 1. Bar charts show the median with IQR. 
A
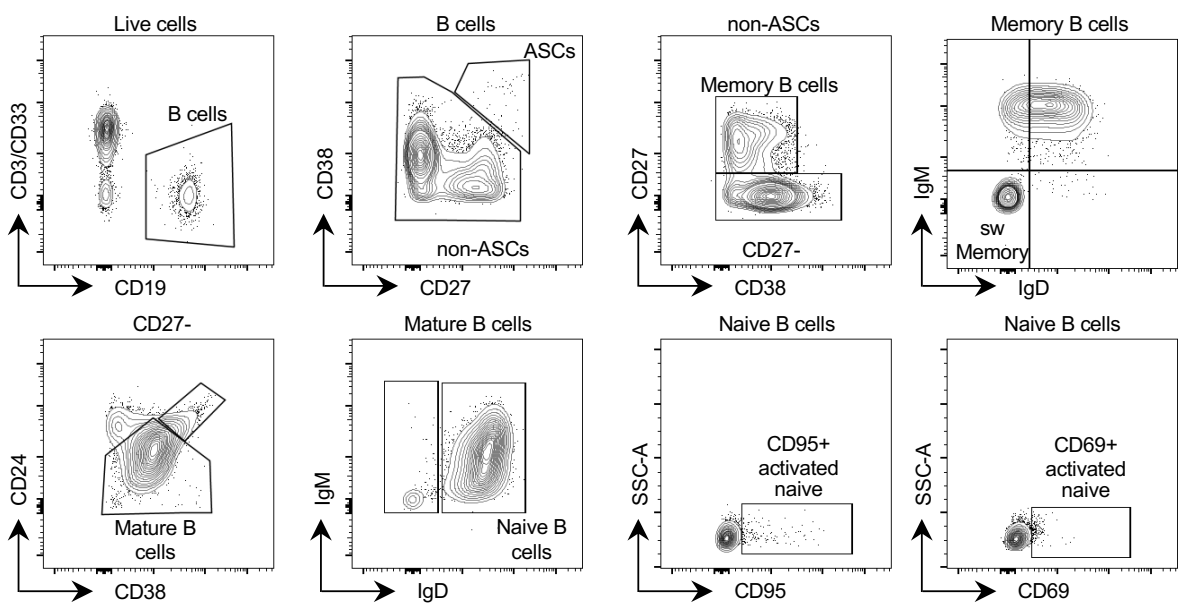

B
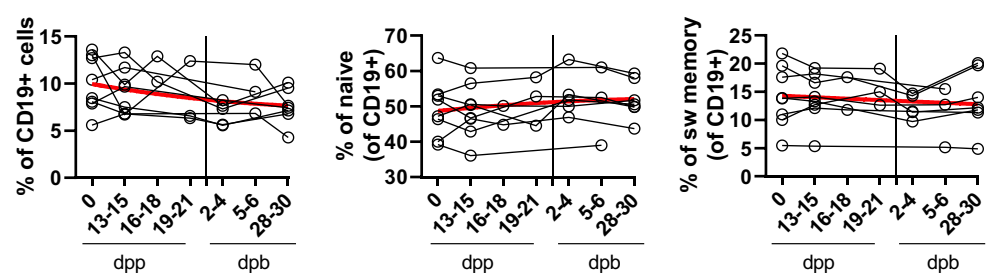

C

anti-SARS-CoV-2 S1 IgM after in vitro re-stimulation and in serum
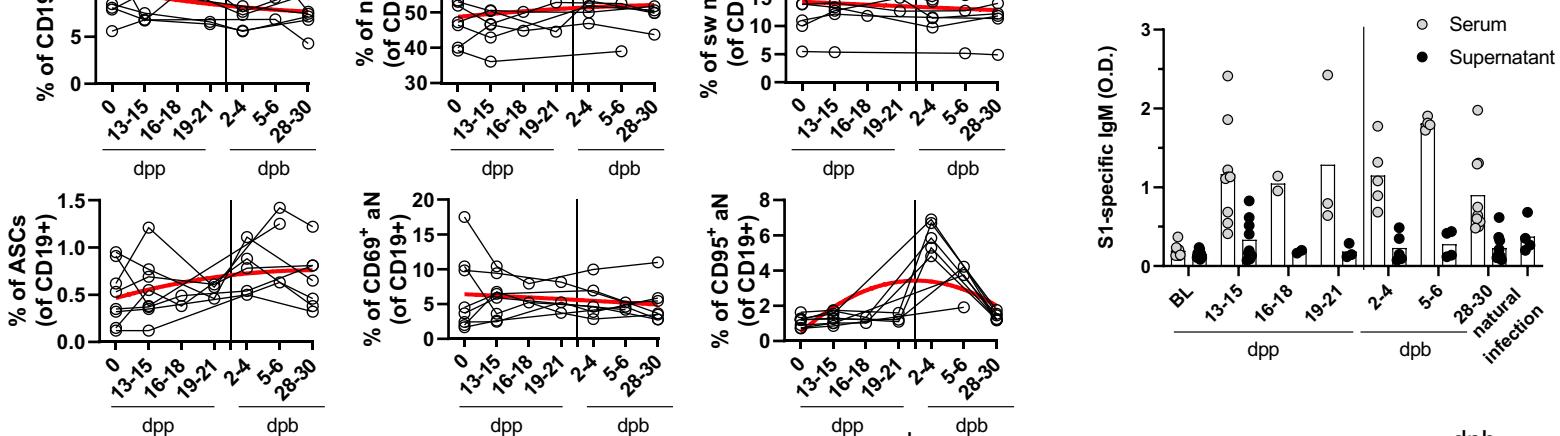

D Neutralizing titers B.1

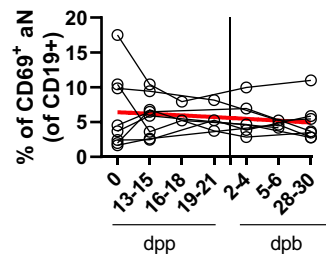

E natural infection
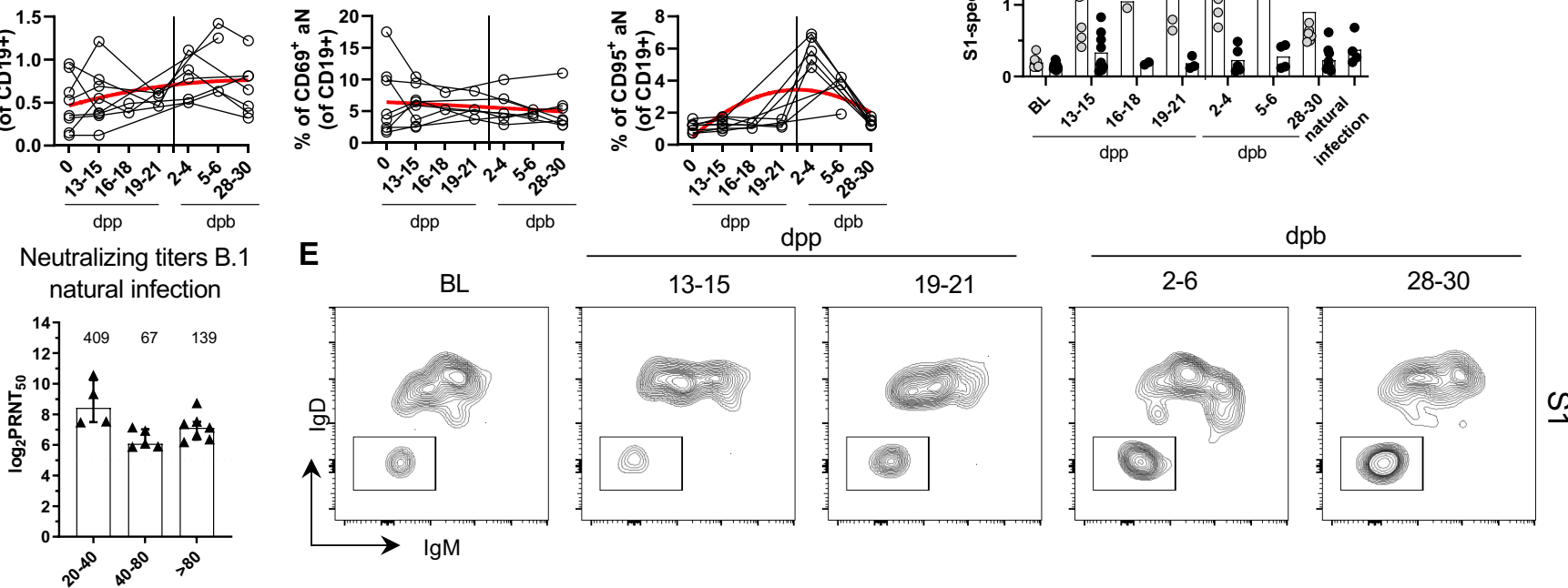

dps
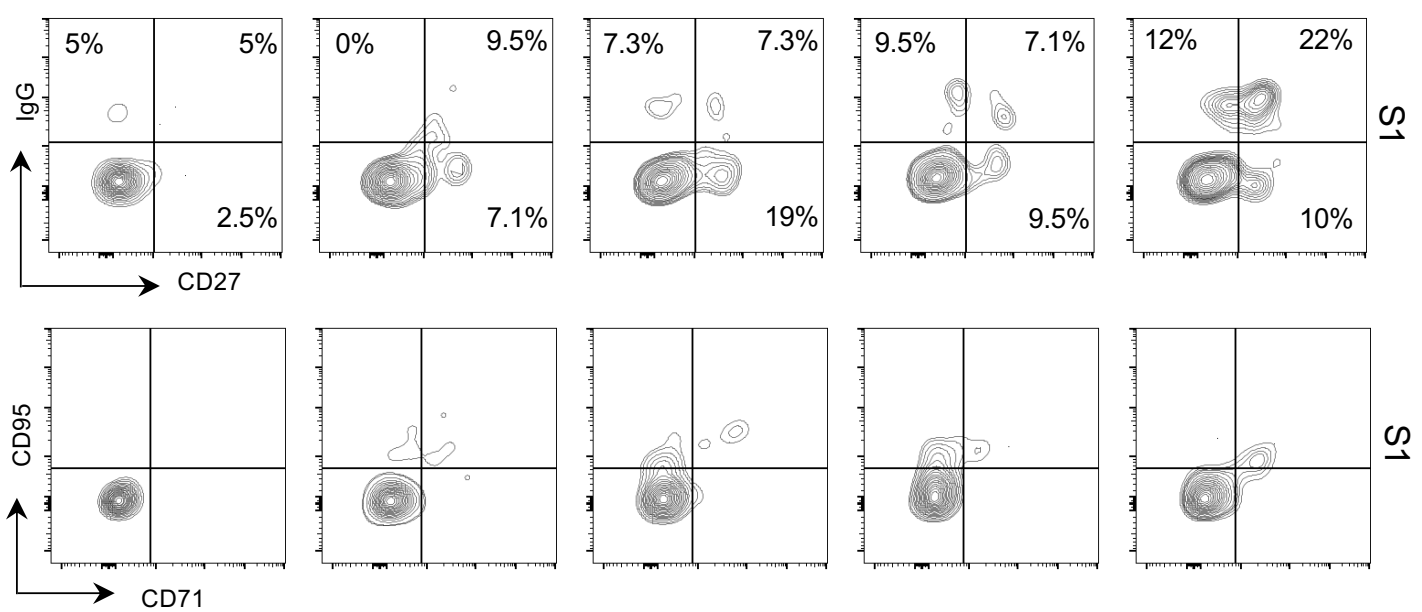

\section{Extended Data Figure 6:}

(A) Gating strategy of flow cytometry data for different B cell subpopulations. (B) \% of CD19+, naïve, switched memory, ASCs, $\mathrm{CD} 69+\mathrm{aN}$ and $\mathrm{CD} 95+\mathrm{aN}$ cells within bulk B cells was determined at BL, post prime/boost. Non-linear fit was calculated in red. (C) Detection of spike IgM in serum at BL, post prime/boost by ELISA. Level of secreted IgM was determined in supernatant of PBMC from BL, post prime/boost and after natural infection after in vitro stimulation for nine days with $\mathrm{CpG}$ and IL-2. (D) Antibody neutralization capacity is depicted as PRNT 50 at different time points post symptom onset in natural SARS-CoV-2 infection with SARS-CoV-2 ancestral variant B.1. Numbers indicate median value (E) Dot plots representing co-expression of IgD/lgM (upper), IgG/CD27 (middle) and CD95/CD71 (lower) on concatenated flies of S1-specific B cells at the indicated time points. ASCs: antibody-secreting cells; sw memory: switched memory; aN: activated naïve; BL: baseline; dpp: days post prime; dpb: days post boost; dps: days post symptoms; O.D.: optical density; PRNT 50 : plaque-reduction neutralization titer 50 . Bar charts show the median with IQR. 
A
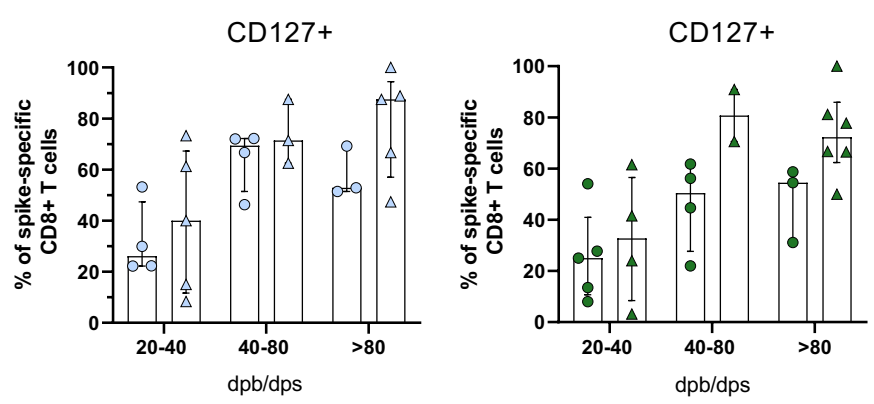
vaccinees
natural infection
- $A^{*} 01 / S_{865}$
$\triangle A^{*} 01 / S_{865}$
- $A^{*} 02 / S_{269}$
$\Delta A^{*} 02 / S_{269}$

B
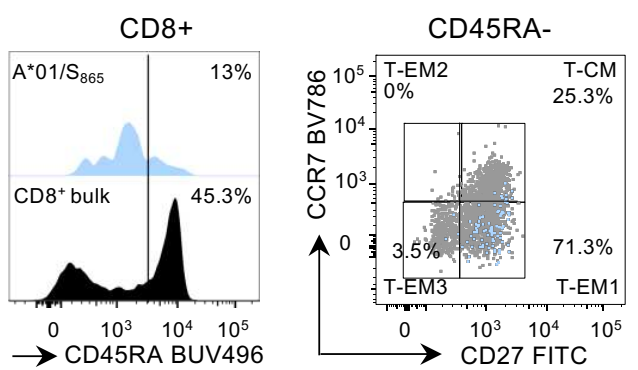

CD45RA+
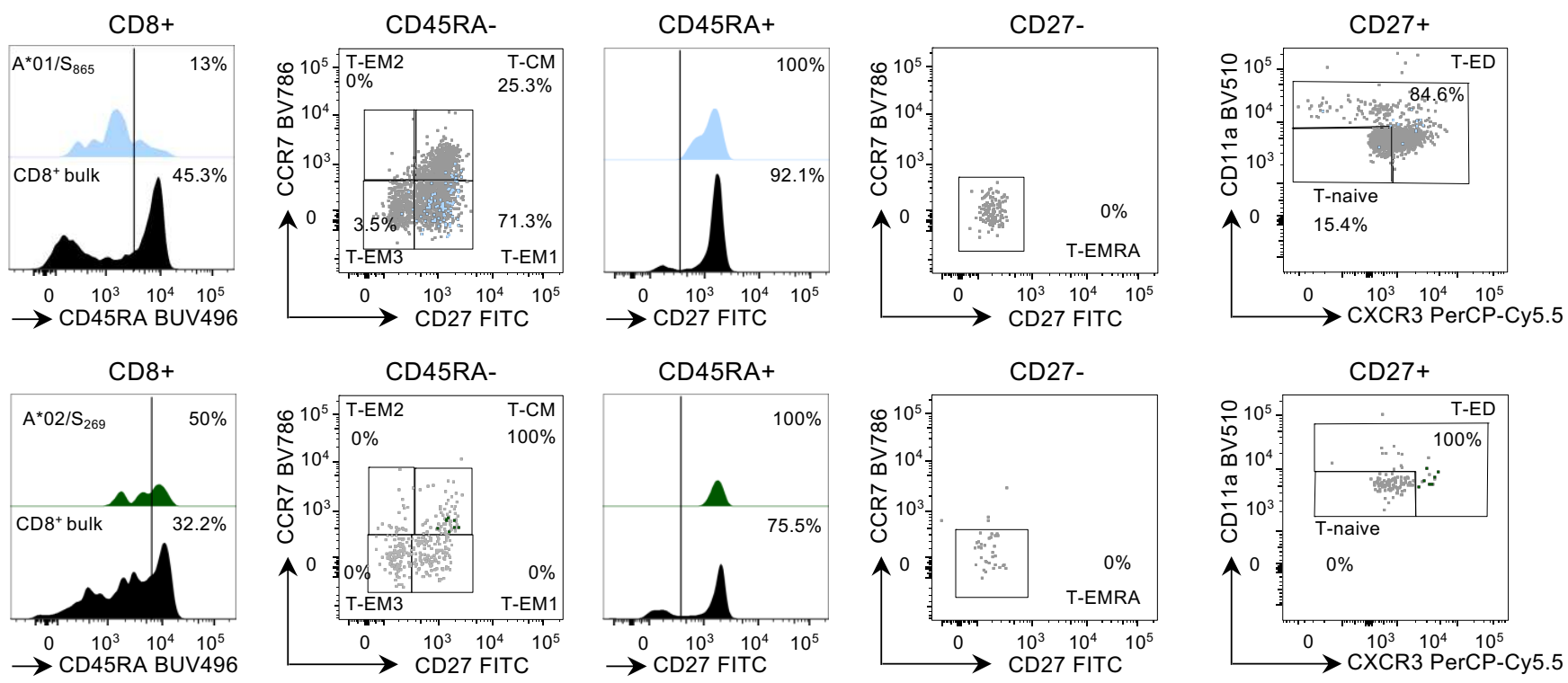

C

T-EM2

T-EM3

TEMRA
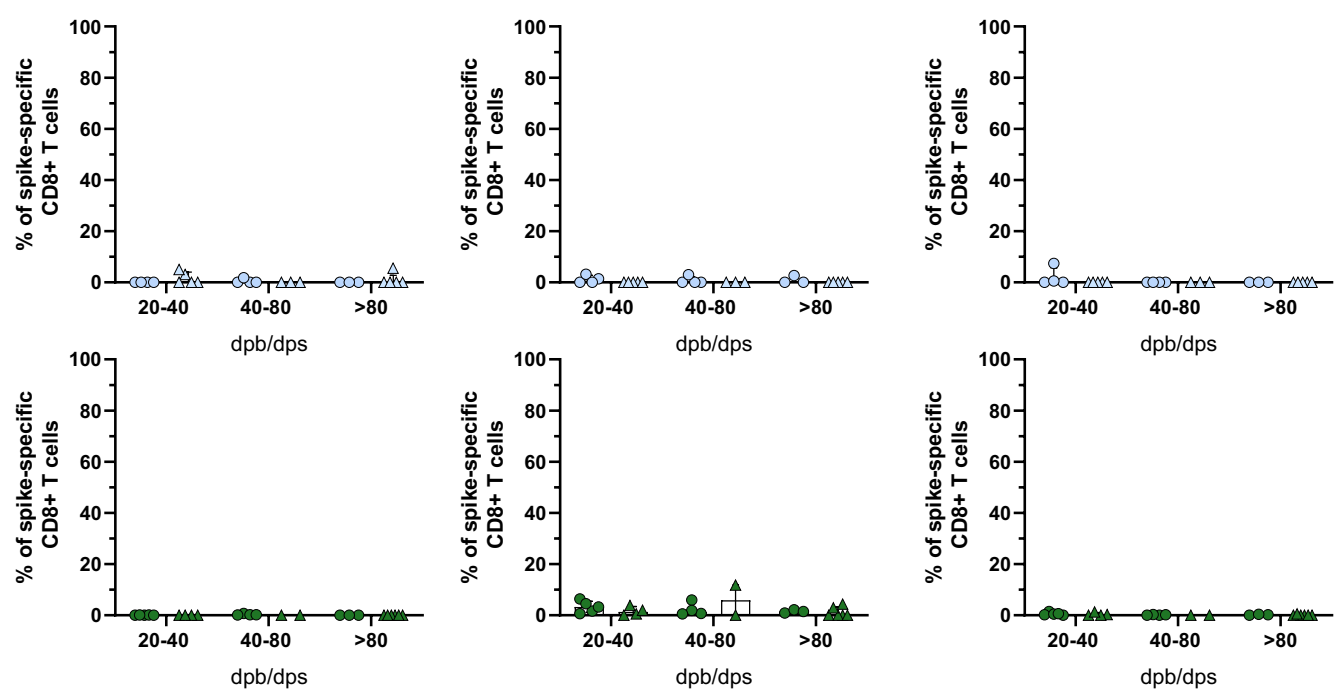

Extended Data Figure 7:

(A) \% of CD127 expressing spike-specific non-naïve CD8+ T cells. (B) Gating strategy of memory CD8+ T cell populations among $A^{*} 01 / S_{865^{-}}$(blue) (28dpb) and $A^{*} 02 / S_{269^{-}}$(green) (98 dps) specific CD8+ T cells, (grey: bulk CD8+ T cells). (C) Distribution of spikespecific CD8+ T-cell memory subsets, TEM2, TEM3 and TEMRA. Bar charts depict the median with IQR. dpb: days post boost; dps: days post symptoms; TEM2: effector memory T cells 2; TEM3: effector memory T cells 3 , TEMRA terminally differentiated effector memory cells re-expressing CD45RA. 

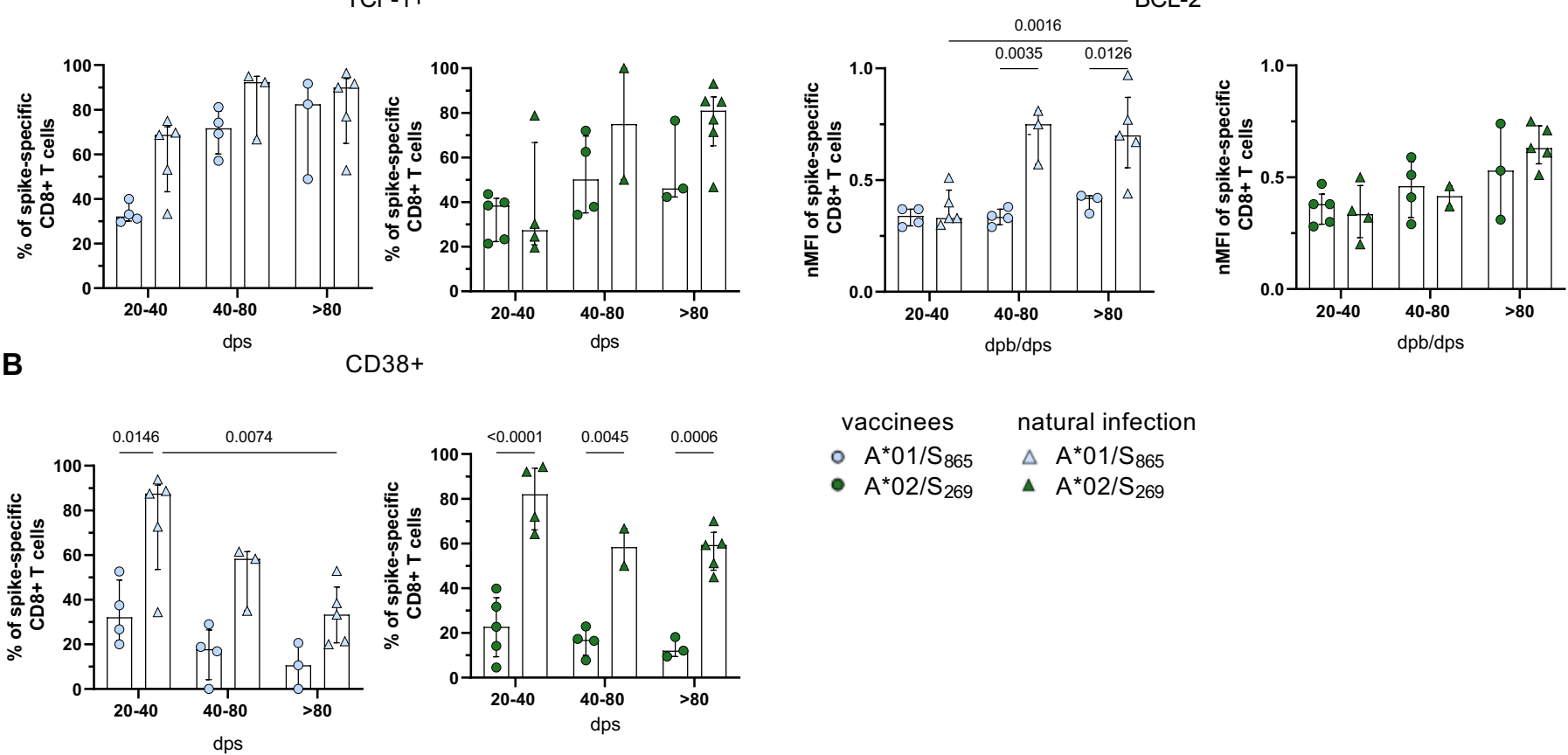

C

Expansion capacity after in vitro expansion

IFN- $\gamma+/$ tet

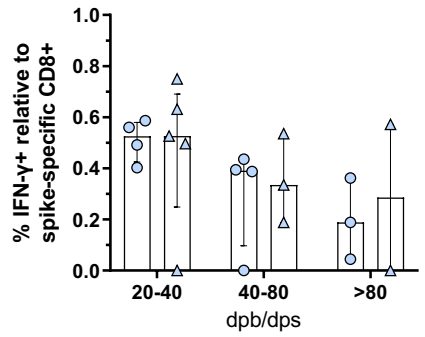

$\mathrm{TNF}+/$ tet

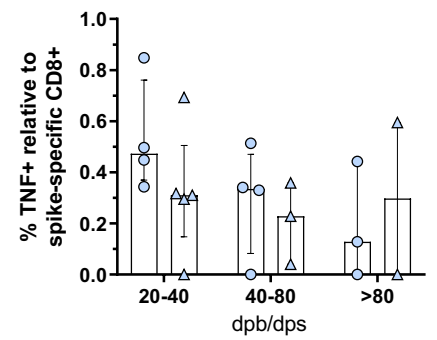

IFN- $-\mathrm{r}+\mathrm{TNF}+/$ tet

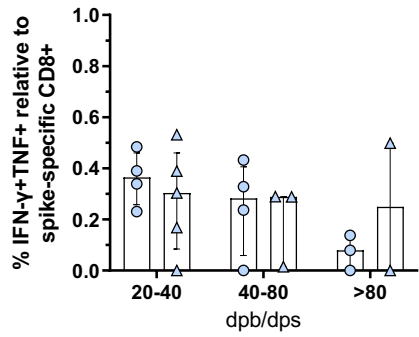

\section{Extended Data Figure 8:}

(A) \% of TCF-1 expressing and nMFI (normalized to naïve CD8+ T cells) of BCL-2 respective spike-specific non-naïve CD8+ T cells. (B) \% of CD38 expressing spike-specific non-naïve CD8+ T cells. (C) Spike-specific CD8+ T cell expansion index of $A^{*} 01 / S_{865}$-specific CD8+ T cells after in vitro expansion. \% of IFN- $\gamma$ - and TNF-producing as well as IFN- $\gamma$ and TNF co-producing CD8+ T cells in relation to the frequency of $A^{*} 01 / S_{865}$-specific CD8+ T cells after in vitro expansion. Bar charts show the median with IQR. $A^{*} 01 / S_{865} n=5$ vaccinees longitudinally and $n=10$ naturally infected patients cross-sectional/longitudinally. $A^{*} 02 / S_{269} n=5$ vaccinees longitudinally and $n=12$ naturally infected patients cross-sectional. 2way ANOVA including Tukey's multiple comparisons test were performed. dpp: days post prime; dps: days post symptoms; t-SNE: t-distributed stochastic neighbor embedding. 


\begin{tabular}{|c|c|c|c|c|c|c|c|c|}
\hline Donor ID & Sex & Age & HLA Type & Cohort & Natural infection & Tested Epitopes & Serum $S 1 \lg G 1$ assay & $\begin{array}{l}\text { Neutralizing Antibodies (Plaque } \\
\text { reduction assay) }\end{array}$ \\
\hline $\mathrm{v} 1$ & $f$ & 45 & $\begin{array}{c}A^{*} 02: 01, A^{*} 33: 03, B^{*} 44: 03, B^{*} 58: 01 \\
\text { DRB1*07:01 }\end{array}$ & vaccinated & & $\begin{array}{l}\text { CD8 T cells: A*02/5269-277 } \\
\text { B cells: } 51, \text { RBD }\end{array}$ & yes & yes \\
\hline v2 & f & 44 & $\begin{array}{c}A^{*} 01: 01, A^{*} 11: 01, B^{*} 15: 17, B^{*} 35: 01 \\
\text { DRB1 } 1^{*} 01: 03, D^{2}: 1^{*} 13: 02\end{array}$ & vaccinated & - & $\begin{array}{l}\text { CD8 T cells: A*01/5865-873 } \\
\text { B cells: } 11, \text { RBD }\end{array}$ & yes & yes \\
\hline v3 & f & 30 & $\begin{array}{c}\mathrm{A}^{*} 01: 01, \mathrm{~A}^{*} 02: 01, \mathrm{~B}^{*} 07: 02, \mathrm{~B}^{*} 37: 01, \\
\text { DRB1*05:01, DRB1*11:03 }\end{array}$ & vaccinated & - & $\begin{array}{c}\text { CD8 T cells: } A^{*} 01 / 5865-873 \quad A^{*} 02 / 5269- \\
277 \\
B \text { ceells: S1, RBD } \\
\end{array}$ & yes & yes \\
\hline $\mathrm{v}_{4}$ & m & 25 & $\begin{array}{c}A^{*} 02: 01, A^{*} 29: 02, B^{*} 45: 01, B^{*} 51: 01 \\
\text { DRB1*04:01, DRB1*13:01 }\end{array}$ & vaccinated & & $\begin{array}{l}\text { CD8 T cells: A*02/5269-277 } \\
\text { B cells: } 51, \text { RBD }\end{array}$ & yes & yes \\
\hline v5 & m & 47 & $\begin{array}{c}A^{*} 01: 01, \mathrm{~A}^{*} 11: 01, \mathrm{~B}^{*} 08: 01, \mathrm{~B}^{*} 15: 01 \\
\text { DRB1*1*01:01, } \mathrm{DRB1}{ }^{*} 03: 01\end{array}$ & vaccinated & - & $\begin{array}{l}\text { CD8 T cells: A*01/5865-873 } \\
\text { B cells: } 51, \text { RBD }\end{array}$ & yes & yes \\
\hline v6 & m & 62 & $\begin{array}{c}A^{*} 01: 01, A^{*} 02: 01, B^{*} 08: 01, B^{*} 15: 01 \\
\text { DRB1*08:01, DRB1*11:01 }\end{array}$ & vaccinated & - & $\begin{array}{c}\text { CD8 T cells: } A^{* 01 / 5865-873} A^{*} 02 / 5269- \\
277 \\
B \text { cells: } 51 \text { RBD }\end{array}$ & yes & yes \\
\hline $\mathrm{V} 7 / \mathrm{HC} 1$ & f & 36 & $\begin{array}{c}A^{*} 01: 01, A^{*} 03: 01, B^{*} 07: 02, B^{*} 57: 01 \\
\text { DRB1 } \\
\text { DR } 07: 01, D R B 1^{*} 15: 01\end{array}$ & $\begin{array}{c}\text { vaccinated / historic } \\
\text { control }\end{array}$ & - & $\begin{array}{l}\text { CD8 T cells: A*01/5865-873 } \\
\text { CD4: DRB1*15:01/ S236-250 } \\
\text { B cells: S1, RBD }\end{array}$ & yes & yes \\
\hline v8 & $\mathrm{m}$ & 31 & $\begin{array}{c}A^{*} 02: 01, B^{*} 15: 01, B^{*} 51: 01, D R B 1^{*} 01: 01 \\
\text { DRB1 }{ }^{*} 11: 01\end{array}$ & vaccinated & - & $\begin{array}{l}\text { CD8 T cells: A*02/5269-277 } \\
\text { B cells: S1, RBD }\end{array}$ & yes & yes \\
\hline vg & $\mathrm{m}$ & 41 & $\begin{array}{c}A^{*} 01: 01, A^{*} 03: 01, B^{*} 08: 01, B^{*} 35: 01 \\
\text { DRB1*1*01:01, DRB1 }\end{array}$ & vaccinated & - & B cells: 11, RBD & no & yes \\
\hline v10 & m & 39 & $\begin{array}{c}\mathrm{A}^{*} 02: 01, \mathrm{~A}^{*} 23: 01, \mathrm{~B}^{*} 13: 02, \mathrm{~B}^{*} 58: 01 \\
\text { DRB1 }\end{array}$ & vaccinated & - & B cells: $51, \mathrm{RBD}$ & no & yes \\
\hline $\mathrm{V} 11 / \mathrm{HC2}$ & $\mathrm{m}$ & 33 & $\mathrm{~A}^{*} 02: 01, \mathrm{~A}^{*} 03: 01, \mathrm{~B}^{*} 07: 02, \mathrm{DRB} 1^{*} 15: 01$ & $\begin{array}{c}\text { vaccinated / historic } \\
\text { control }\end{array}$ & - & CD4 T cells: DRB1*15:01/ S236-250 & no & yes \\
\hline $\mathrm{V} 12 / \mathrm{HC3}$ & $\mathrm{m}$ & 41 & $\begin{array}{c}A^{*} 01: 01, \mathrm{~A}^{*} 02: 01, \mathrm{~B}^{*} 08: 01, \mathrm{~B}^{*} 40: 01 \\
\text { DRB1 } 1^{*} 14: 02, \mathrm{DRB} 1^{*} 15: 01\end{array}$ & $\begin{array}{c}\text { vaccinated / historic } \\
\text { control }\end{array}$ & - & CD4T cells: DRB1*15:01/ S236-250 & no & yes \\
\hline v13 & f & 37 & $\begin{array}{c}A^{*} 02: 01, A^{*} 68: 01, B^{*} 15: 01, B^{*} 40: 01 \\
\text { DRB1*13:13:01, DRB1*15:01 }\end{array}$ & vaccinated & - & CD4 T cells: DRB1*15:01/ S236-250 & no & yes \\
\hline v14 & $\mathrm{m}$ & 35 & $\begin{array}{c}A^{*} 01: 01, A^{*} 26: 01, B^{*} 07: 02, B^{*} 27: 05 \\
\text { DRB1 } \\
\text { * } 01: 01, \text { DRB1 }\end{array}$ & vaccinated & - & CD4 T cells: DRB1*15:01/ s236-250 & no & yes \\
\hline V15 & $\mathrm{m}$ & 35 & $\begin{array}{c}A^{* 01} 01: 01, A^{*} 24: 02, B^{*} 07: 02, B^{*} 27: 05 \\
\text { DRB1 } 1^{*} 15: 01, \quad D R B 1^{*} 16: 01\end{array}$ & vaccinated & - & CD4 T cells: DRB1*15:01/ 5236-250 & no & yes \\
\hline $\mathrm{V} 16 / \mathrm{HC} 4$ & f & 60 & $\begin{array}{c}A^{*} 02: 01, B^{*} 07: 02, B^{*} 44: 02, D R B 1^{*} 04: 01 \\
\text { DRB1 }\end{array}$ & \begin{tabular}{|c|} 
vaccinated / historic \\
control
\end{tabular} & - & CD4T cells: DRB1*15:01/ S236-250 & no & yes \\
\hline v17 & $\mathrm{m}$ & 40 & $\begin{array}{c}A^{*} 02: 01, A^{*} 03: 01, B^{*} 07: 02, B^{*} 57: 01 \\
\operatorname{DRB}^{*} 07: 01,0 R B 1^{*} 15: 01\end{array}$ & vaccinated & - & CD4 T cells: DRB1*15:01/ S236-250 & no & yes \\
\hline HC5 & $\mathrm{m}$ & 33 & $\begin{array}{c}A^{*} 24: 02, A^{*} 29: 02, B^{*} 07: 02, B^{*} 44: 03 \\
\text { DRB1 } \\
\text { *14:54, DRB1*15:01 }\end{array}$ & historic control & & CD4 T cells: DRB1*15:01/ S236-250 & no & no \\
\hline HC6 & $\mathrm{m}$ & 33 & $\begin{array}{c}A^{*} 02: 01, A^{*} 03: 01, B^{*} 07: 02, D R B 1 * 04: 01 \\
\text { DRB1 }{ }^{*} 15: 01\end{array}$ & historic control & - & CD4 T cells: DRB1*15:01/ S236-250 & no & no \\
\hline $\mathrm{HC7}$ & $\mathrm{m}$ & 30 & $\begin{array}{c}A^{*} 01: 01, A^{*} 03: 01, B^{*} 08: 01, B^{*} 35: 03 \\
\operatorname{DRB1}^{*} 03: 01, \mathrm{DRB}^{*} 15: 01\end{array}$ & historic control & - & CD4 T cells: DRB1*¹5:01/ S236-250 & no & no \\
\hline HC8 & $f$ & 35 & $\begin{array}{c}A^{*} 02: 01, \mathrm{~A}^{*} 26: 01, \mathrm{~B}^{*} 07: 02, \mathrm{~B}^{*} 38: 01 \\
\text { DRB1 } 1^{* 13} 13: 01, \mathrm{DRB} 1^{*} 15: 01\end{array}$ & historic control & & CD4 T cells: DRB1*15:01/ S236-250 & no & no \\
\hline 11 & $f$ & 86 & $\begin{array}{c}A^{*} 02: 01, A^{*} 31: 01, B^{*} 13: 02, B^{*} 40: 01 \\
\text { DRB1*04:01, }\end{array}$ & acute & mild infection & CD8 T cells: A*02/5269-277 & yes & no \\
\hline 12 & $\mathrm{~m}$ & 63 & $\begin{array}{c}A^{*} 02: 01, B^{*} 44: 02, B^{*} 58: 01, D R B 1^{*} 03: 01 \\
\text { DRB1 }{ }^{*} 13: 01\end{array}$ & acute & mild infection & CD8 T cells: $A^{*} 02 / 5269-277$ & yes & no \\
\hline 13 & $\mathrm{~m}$ & 69 & $\begin{array}{c}\mathrm{A}^{*} 01: 01, \mathrm{~A}^{*} 25: 01, \mathrm{~B}^{*} 08: 01, \mathrm{~B}^{*} 18: 01 \\
\text { DRB1 }\end{array}$ & acute / resolved & mild infection & CD8 T cells: A*01/5865-873 & yes & yes \\
\hline 14 & $f$ & 39 & $\begin{array}{c}\mathrm{A}^{*} 01: 01, \mathrm{~A}^{*} 30: 02, \mathrm{~B}^{*} 18: 01, \mathrm{~B}^{*} 41: 01 \\
\mathrm{DRB} 1^{*} 07: 01, \mathrm{DRB} 1^{*} 07: 11\end{array}$ & acute / resolved & mild infection & CD8 T cells: A*01/5865-873 & yes & yes \\
\hline 15 & $\mathrm{~m}$ & 34 & $\mathrm{~A}^{*} 01: 01, \mathrm{~A}^{*} 03: 01, \mathrm{~B}^{*} 08: 01, \mathrm{DRB} 1^{*} 03: 01$ & acute / resolved & mild infection & CD8 T cells: A*01/5865-873 & yes & yes \\
\hline 16 & $\mathrm{~m}$ & 57 & $\begin{array}{c}A^{*} 02: 01, A^{*} 24: 02, B^{*} 44: 02, B^{*} 57: 01 \\
\text { DR1 }\end{array}$ & acute / resolved & mild infection & CD8 T cells: A*02/5269-277 & yes & no \\
\hline 17 & $\mathrm{~m}$ & 37 & $\begin{array}{c}A^{*} 01: 01, A^{*} 29: 02, B^{*} 07: 02, B^{*} 40: 01 \\
\text { DRB1*01:03, DRB1*11:01 }\end{array}$ & resolved & mild infection & CD8 T cells: $A^{*} 01 / 5865-873$ & yes & yes \\
\hline 18 & $\mathrm{~m}$ & 36 & $\begin{array}{c}A^{*} 01: 01, A^{*} 03: 01, B^{*} 37: 01, B^{*} 40: 01 \\
\text { DRB1 } 1^{*} 13: 02, \quad D R B 1^{*} 15: 01\end{array}$ & resolved & mild infection & CD8 T cells: A*01/5865-873 & yes & ves \\
\hline 19 & $f$ & 46 & $\begin{array}{c}A^{*} 02: 01, A^{*} 11: 01, B^{*} 07: 02, B^{*} 49: 01 \\
\text { DRB1*04 }\end{array}$ & resolved & mild infection & CD8 T cells: A*02/5269-277 & no & no \\
\hline 110 & $\mathrm{~m}$ & 30 & $\begin{array}{c}A^{*} 01: 01, A^{*} 02: 01, B^{*} 15: 01, B^{*} 51: 01 \\
\text { DRB1 } 1^{*} 11: 01, D R B 1^{*} 13: 01\end{array}$ & resolved & mild infection & $\begin{array}{c}\text { CD8 T cells: } A^{*} 01 / 5865-873 A^{*} 02 / 5269- \\
277\end{array}$ & yes & yes \\
\hline 111 & $\mathrm{~m}$ & 29 & $\begin{array}{c}A^{*} 01: 01, A^{*} 31: 01, B^{*} 39: 01, B^{*} 51: 01 \\
\text { DRB1 } 1^{*} 01: 01, \quad D B B 1^{*} 12: 01\end{array}$ & resolved & mild infection & CD8 T cells: A*01/5865-873 & yes & no \\
\hline 112 & $\mathrm{~m}$ & 56 & $\begin{array}{c}A^{*} 01: 01, A^{*} * 3: 01, B^{*} 07: 02, B^{*} 08: 01, \\
D R B 1^{*} 03: 01, D R B 1^{*} 15: 01\end{array}$ & resolved & mild infection & CD8 T cells: A*01/5865-873 & yes & yes \\
\hline 113 & $\mathrm{~m}$ & 38 & $\begin{array}{c}A^{*} 02: 01, A^{*} 31: 01, B^{*} 07: 02, B^{*} 15: 01 \\
\operatorname{DRB}^{*} 12: 01, \mathrm{DRB}^{*} 15: 01\end{array}$ & resolved & mild infection & CD8 T cells: A*02/S269-277 & yes & no \\
\hline 114 & $\mathrm{~m}$ & 27 & $\begin{array}{c}A^{*} 02: 01, A^{*} 31: 01, B^{*} 07: 02, B^{*} 15: 01 \\
\text { DRB1 } 1^{*} 12: 01, D R B 1^{*} 15: 01\end{array}$ & resolved & mild infection & CD8 T cells: A*02/S269-277 & yes & no \\
\hline 115 & $\mathrm{~m}$ & 22 & $\begin{array}{c}\mathrm{A}^{*} 02: 01, \mathrm{~B}^{*} 15: 01, \mathrm{~B}^{*} 40: 01, \mathrm{DRB} 1^{*} 08: 01, \\
\text { DRB1 }{ }^{*} 13: 02\end{array}$ & resolved & mild infection & CD8 T cells: $A^{*} 02 / 5269-277$ & yes & no \\
\hline 116 & $f$ & 43 & $\begin{array}{c}\mathrm{A}^{*} 02: 01, \mathrm{~A}^{*} 31: 01, \mathrm{~B}^{*} 27: 02, \mathrm{~B}^{*} 39: 01 \\
\text { DRB1*12:01, DRB1*16:01 }\end{array}$ & resolved & mild infection & CD8 T cells: A*02/S269-277 & yes & no \\
\hline 117 & $f$ & 32 & $\begin{array}{c}A^{*} 02: 01, B^{*} 18: 01, B^{*} 38: 01, D R B 1^{*} 07: 01 \\
\text { DRB1 }{ }^{*} 13: 01\end{array}$ & resolved & mild infection & CD8 T cells: A*02/5269-277 & yes & no \\
\hline 118 & $f$ & 42 & $\begin{array}{c}A^{*} 01: 01, A^{*} 32: 01, B^{*} 07: 02, B^{*} 40: 02 \\
\text { DRB1 } 1^{*} 07: 01, D R B 1^{*} 13: 01\end{array}$ & resolved & mild infection & CD8 T cells: A*01/5865-873 & yes & yes \\
\hline 119 & $f$ & 32 & $\begin{array}{c}A^{*} 02: 01, \mathrm{~A}^{*} 03: 01, \mathrm{~B}^{*} 40: 01, \mathrm{~B}^{*} 44: 02 \\
\text { DRB1 } 1^{* 11}: 04, \mathrm{DRB} 1^{*} 13: 01\end{array}$ & resolved & mild infection & CD8 T cells: $A^{*} 02 / 5269-277$ & yes & yes \\
\hline 120 & f & 62 & $\begin{array}{c}A^{*} 02: 01, A^{*} 32: 01, B^{*} 27: 05, B^{*} 51: 01 \\
\text { DRB1*11:01, } \\
\text { DRB1*15:01 }\end{array}$ & resolved & mild infection & CD8 T cells: A*02/5269-277 & yes & yes \\
\hline 121 & $f$ & 48 & $\begin{array}{c}\mathrm{A}^{*} 02: 01, \mathrm{~A}^{*} 03: 01, \mathrm{~B}^{*} 07: 02, \mathrm{~B}^{*} 13: 02 \\
\mathrm{DRB} 1^{*} 07: 01, \mathrm{DRB1} 1^{*} 13: 05\end{array}$ & resolved & mild infection & CD8 T cells: $A^{*} 02 / 5269-277$ & yes & yes \\
\hline 122 & $f$ & 36 & $\begin{array}{c}A^{*} 01: 01, \mathrm{~A}^{*} 03: 01, \mathrm{~B}^{*} 07: 02, \mathrm{~B}^{*} 08: 01 \\
\text { DRB1 } 1^{*} 03: 01, \mathrm{DRB} 1^{*} 04: 01\end{array}$ & resolved & mild infection & CD8 T cells: A*01/5865-873 & yes & no \\
\hline
\end{tabular}


A

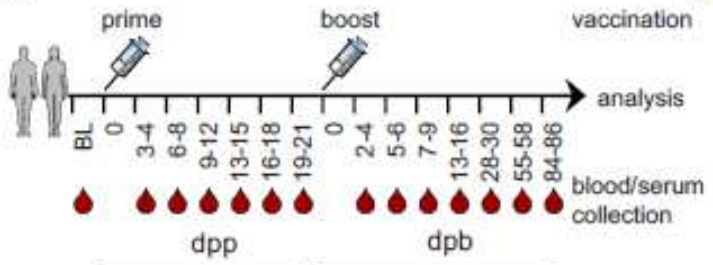

C Spike-specific $\mathrm{CD} 8+\mathrm{T}$ cells calc. ex vivo frequency $\quad \rightarrow-A^{*} 01 / S_{365}$

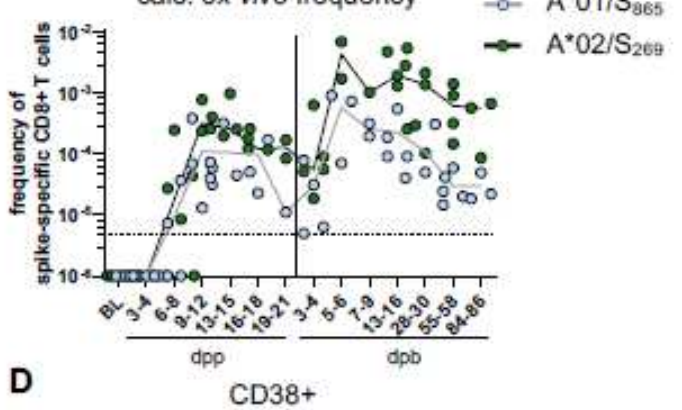

CD38+

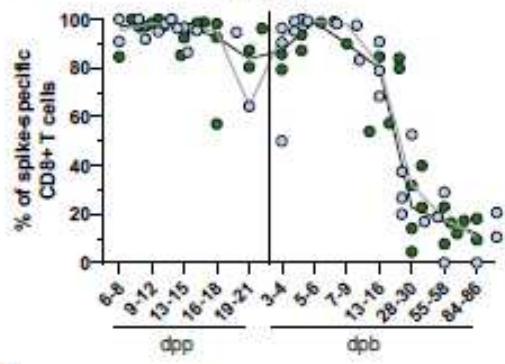

E
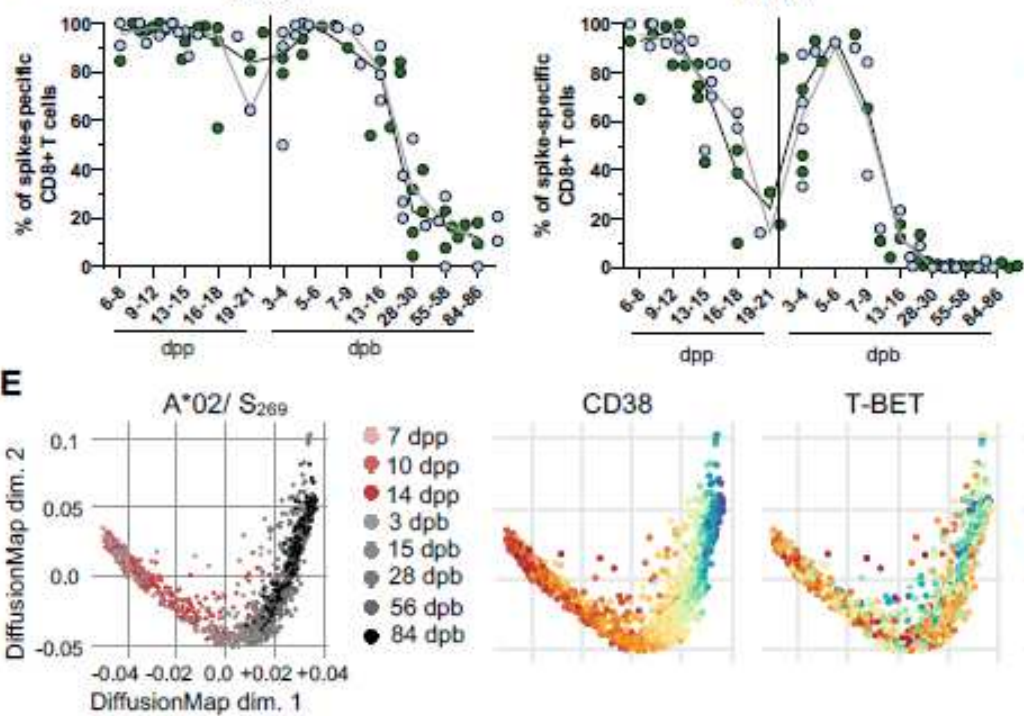

CD38

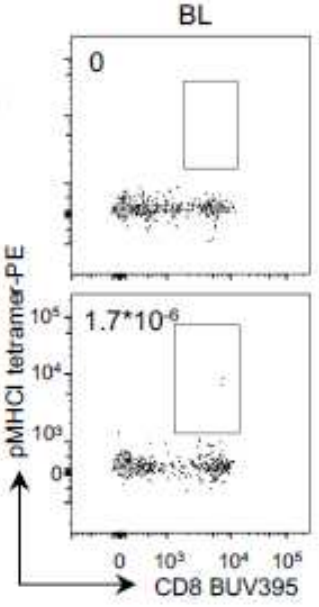

ex vivo spike-specific $C D 8+T$ cells (enriched)
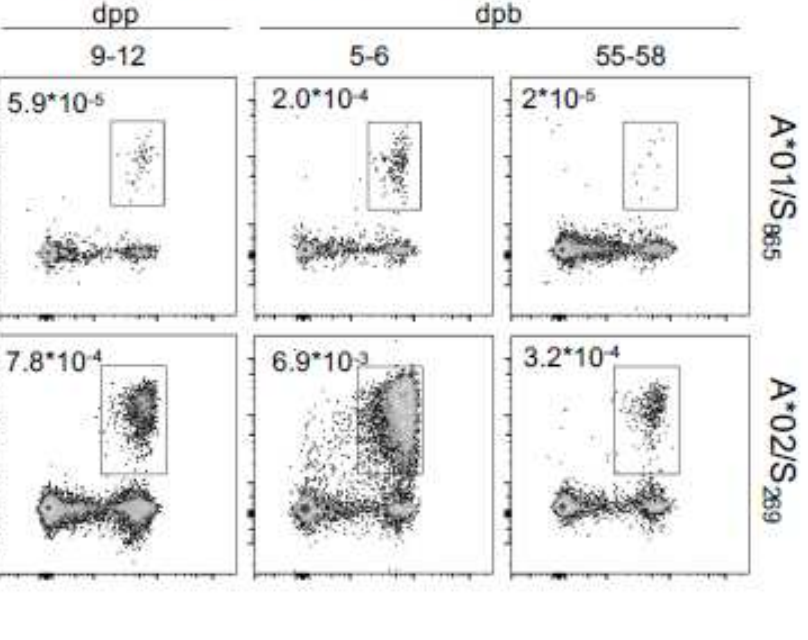

T-BETH

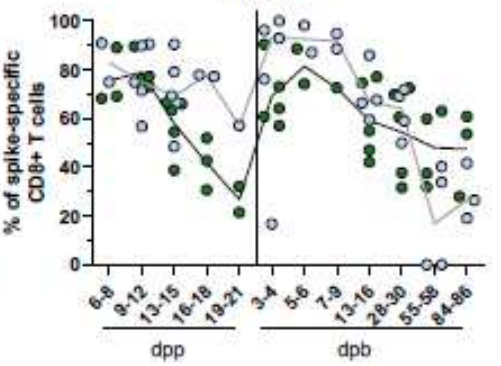

scaled

TOX

$\mathrm{BCL}-2$
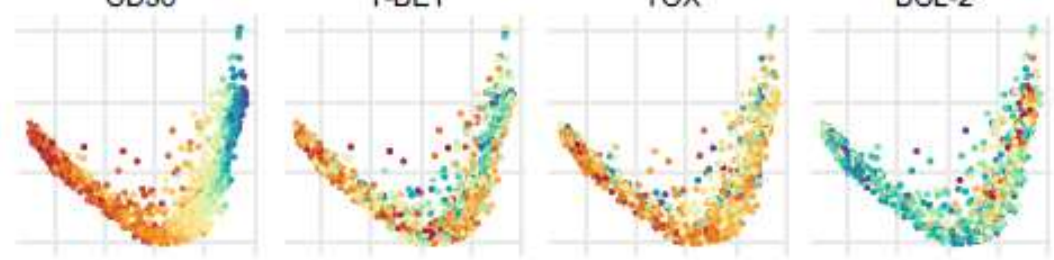

G calc. ex vivo frequencies

TCF-1

Spike-specific TCF-1+CD8+T

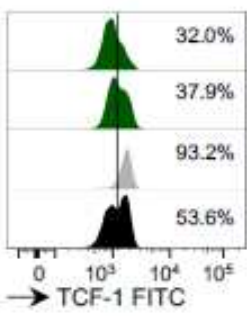
cells

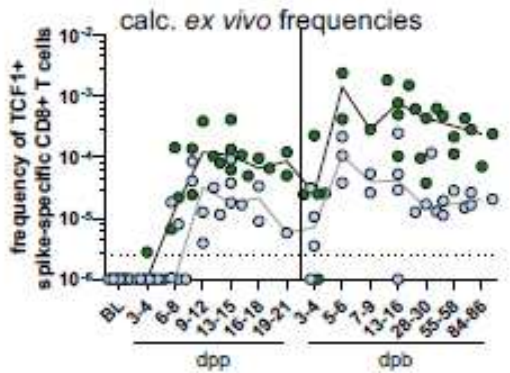

Figure 1

Bnt162b2 vaccine-elicited epitope-specific CD8+ T cells: early induction, vigorous Teff response and robust establishment of memory precursors $(A)$ Timeline showing blood and serum collection before and after prime and boost vaccination. (B) Dot plots showing $A^{*} 01 / \mathrm{S} 865$-and $A^{*} 02 / S 269-$ specific CD8+ T 
cells ex vivo after $\mathrm{pMHCl}$ tetramer-based enrichment at $\mathrm{BL}$, before and after boost vaccination. (C) The calculated ex vivo frequency is indicated at $B L, d p p$ and dpb for $A * 01 / S 865$ - and $A * 02 / S 269$-specific CD8+ T cells. (D) \% of CD38, Ki-67 and T-BEThi expressing A*01/S865- and A*02/S269-specific non-naïve CD8+ T cells. (E) Diffusion map showing flow cytometry data for $A^{\star} 02 / S 269-s p e c i f i c ~ C D 8+T$ cells in relation to dpp (shades of red) and dpb (shades of grey) in one individual. CD38, T-BET, TOX and BCL-2 expression levels are plotted on the diffusion map (color-code: blue, low expression; red, high expression). (F-G) Exemplary histograms depicting the expression levels of CD127 and TCF-1 in A*02/S269- (green) specific, naïve (grey) and bulk (black) CD8+ T cells 14-16 and 55-59 dpb. Calculated ex vivo frequencies of non-naïve spike-specific CD8+ T cells expressing CD127 or TCF- 1 for $A * 01 / S 865$ - and $A * 02 / S 269$ specific CD8+ T cells. BL: baseline; dpp: days post prime; dpb: days post boost. 
A

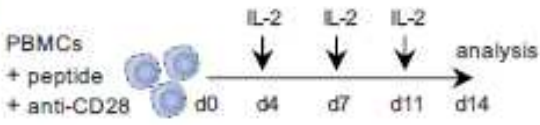

B

B d14 after in vitro expansion

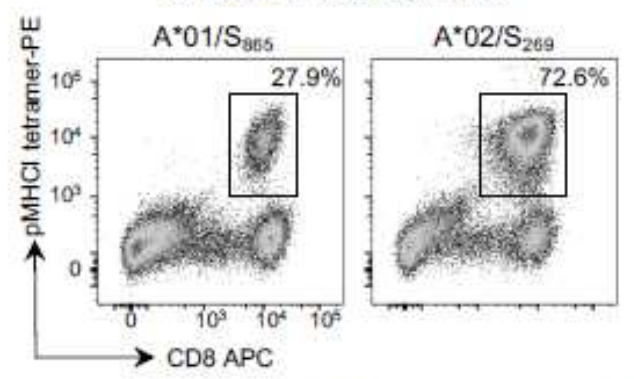

E

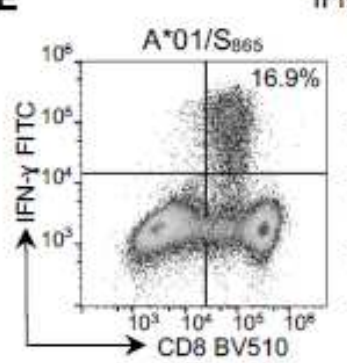

IFN- $\gamma^{+}$

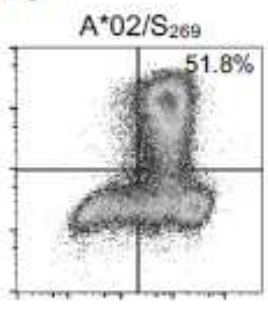

IFN- $\gamma+/$ tet

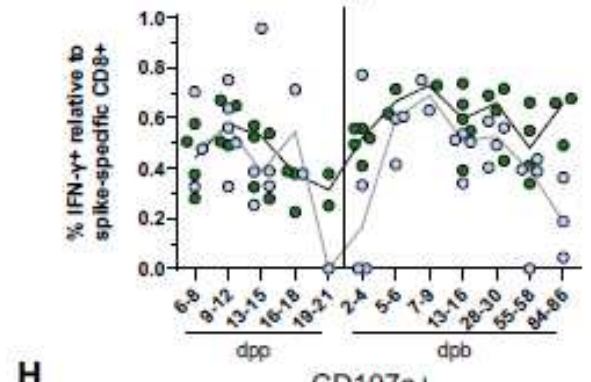

H

CD107a+

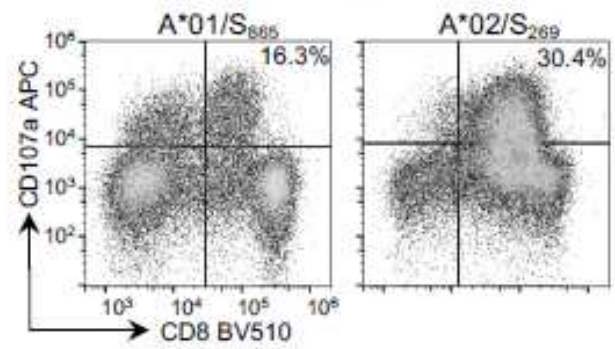

C

Frequency after in vitro expansion

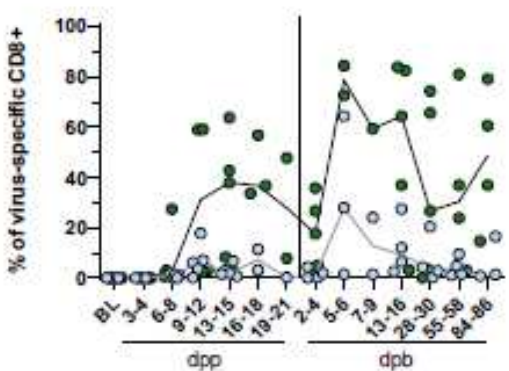

F

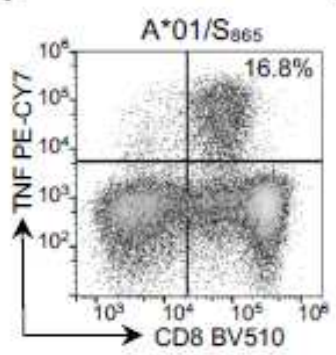

TNF+

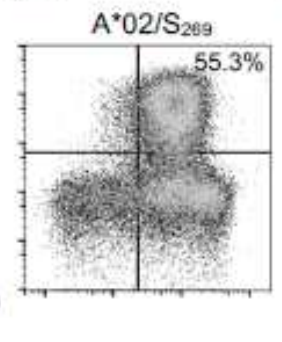

$\mathrm{TNF}+$ / tet
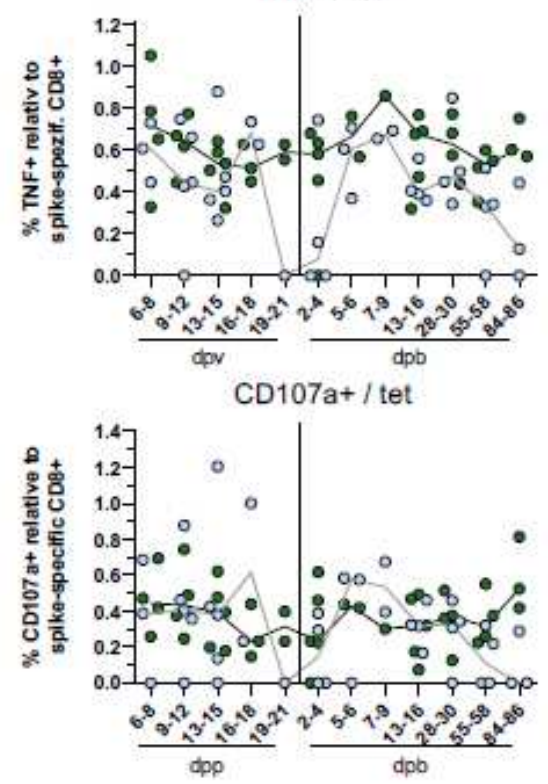

D

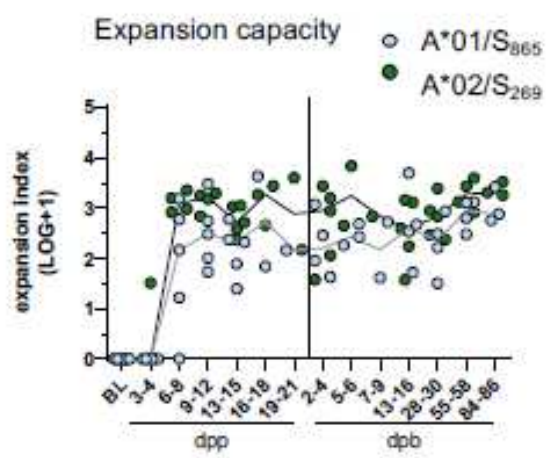

G

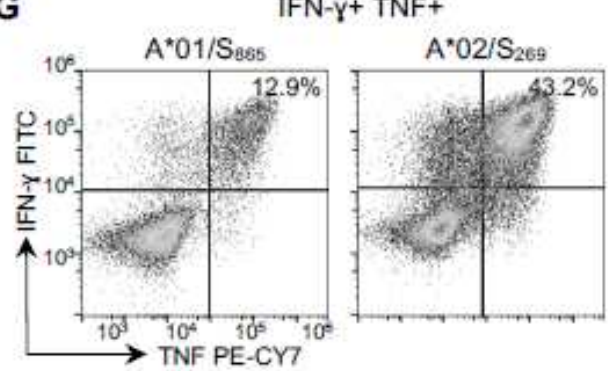

IFN- $\mathrm{\gamma}+\mathrm{TNF}+$ / tet

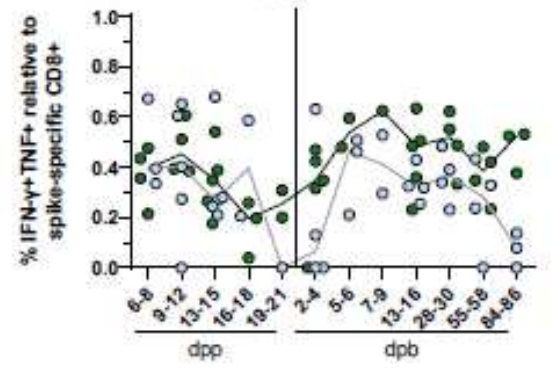

Figure 2

Proper and conserved functional capacities of circulating spike-specific CD8+ T cells following prime and boost vaccination (A) Workflow depicting peptide-specific in vitro expansion of CD8+ T cells. (B) Dot plots showing $A * 01 / S 865$ - and $A * 02 / S 269$-specific CD8+ T cells after in vitro expansion. (C, D) Frequency and expansion capacity of the respective spike-specific CD8+ T cells after in vitro expansion. (E-H) Dot plots showing IFN- $\gamma$ - and TNF-, IFN- $\gamma$ /TNF- and CD107a-expressing CD8+ T cells after in vitro expansion. \% of CD8+ $T$ cells producing the respective cytokines in relation to the frequency of $A * 01 / S 865-$ and 
A*02/S269-specific CD8+ T cells was determined. Dot plot show samples 5-6 dpb. BL: baseline; dpp: days post prime; dpb: days post boost.
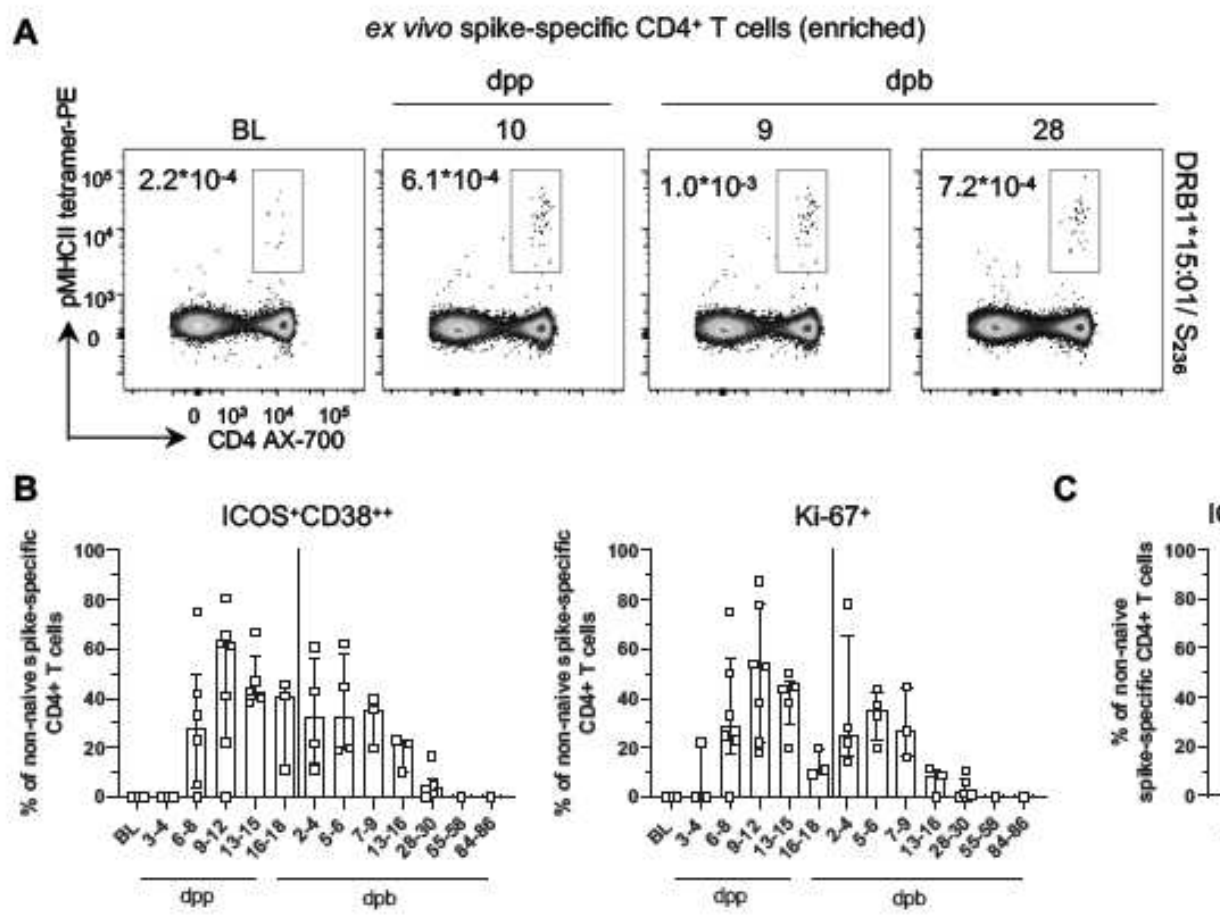

Spike-specific CD4*T cells caic. ex vivo frequency

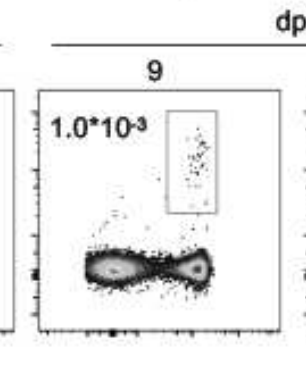

C
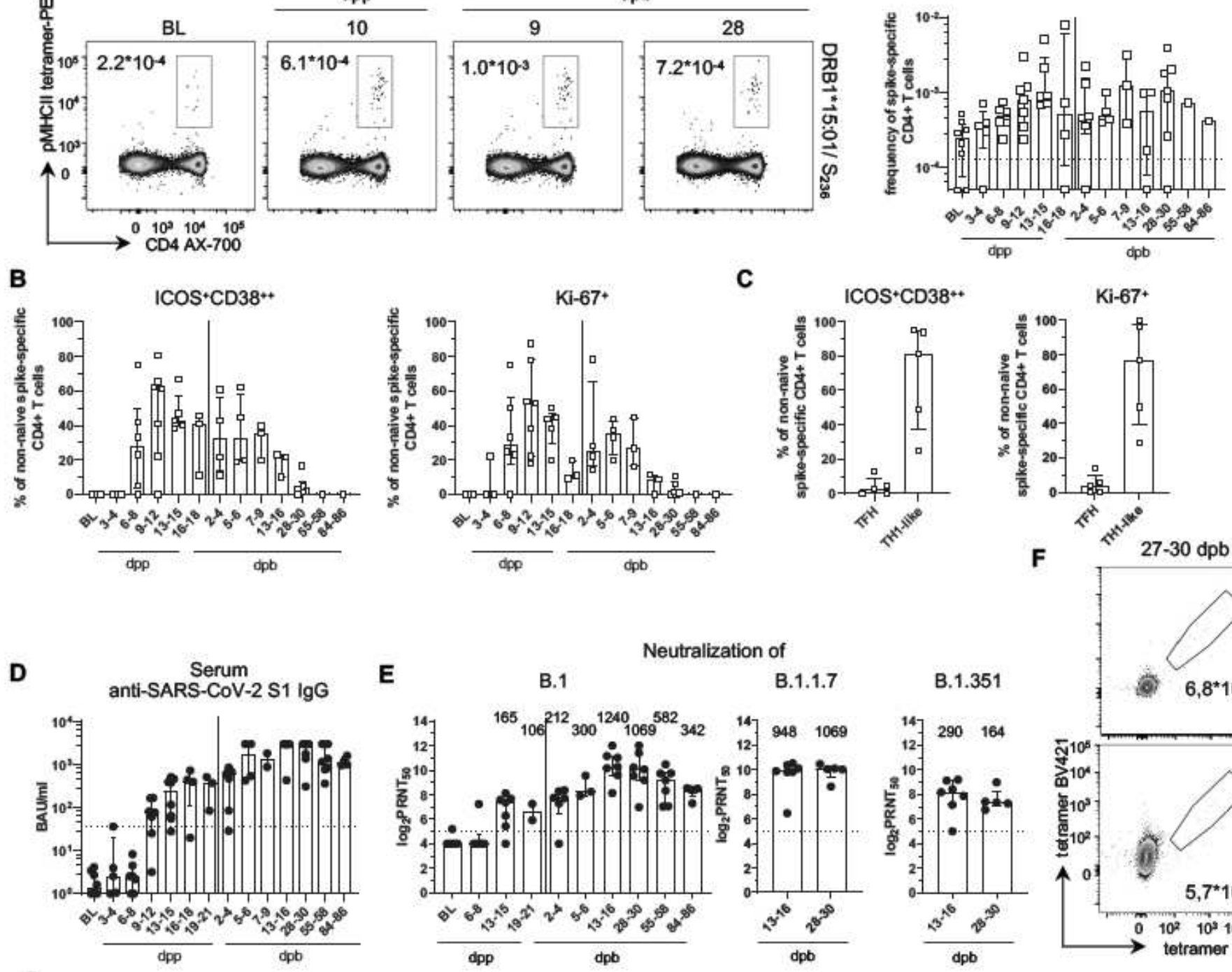

B.1.1.7

B.1.351
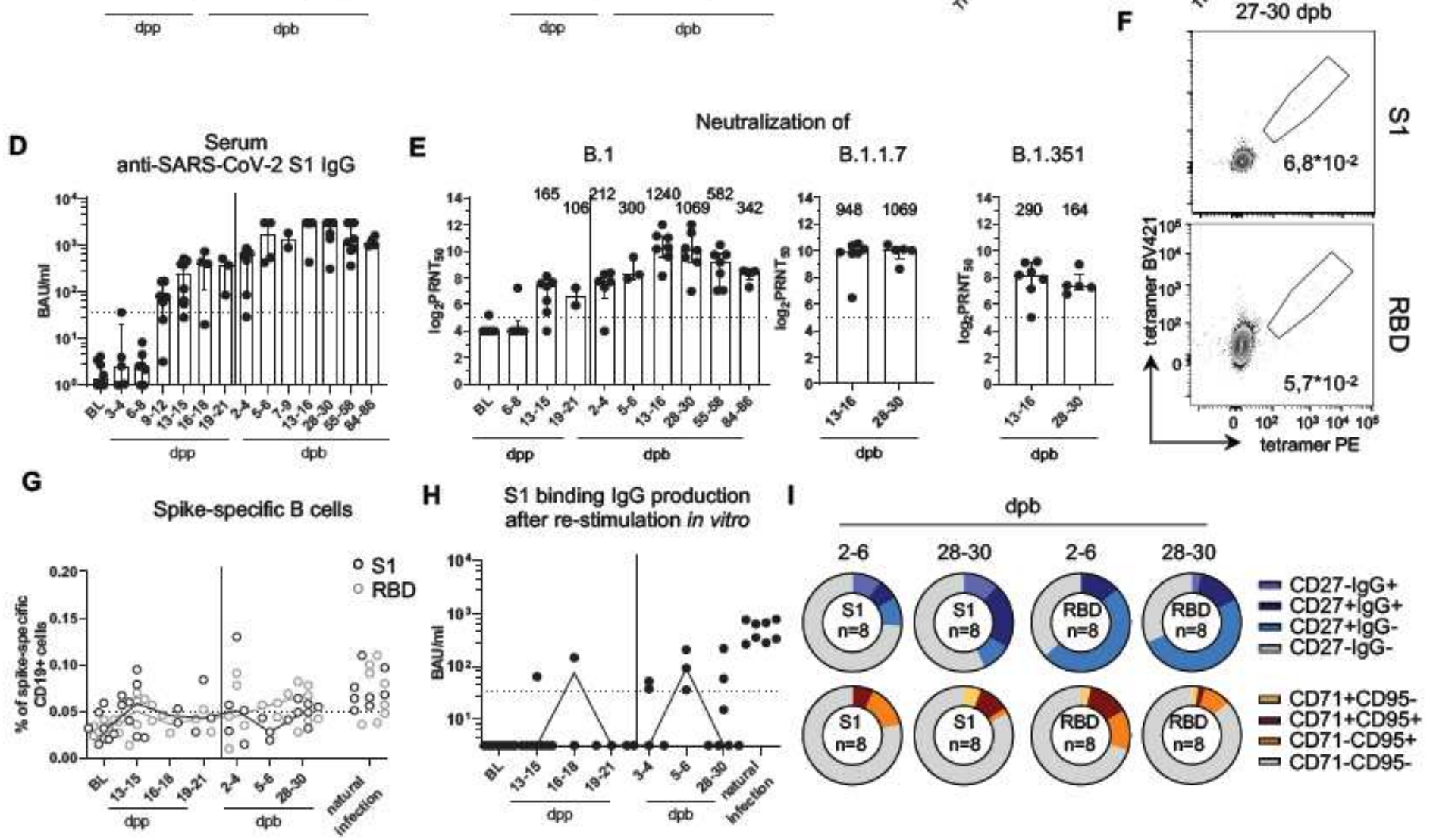

Figure 3

Delayed appearance of circulating spike-specific CD4+T cells, B cells and antibodies (A) Dot plots showing DRB1*15:01/ S236-specific CD4+ T cells ex vivo after pMHCll tetramer-based enrichment at BL and after prime/ boost. The bar graph shows the calculated ex vivo frequency of DRB1*15:01/S236- 
specific CD4+ T cells. (B) ICOS+CD38++ and Ki-67 expression within non-naïve, DRB1*15:01/S236specific CD4+ T cells (C) ICOS+CD38++ and Ki-67-expressing non-naïve DRB1*15:01/S236-specific CD4+ T cells on 9-2 dpp within the population of TFH (CXCR5+PD-1+) and TH1 (CXCR5-CXCR3+) cells. (D) AntiSARS-CoV-2 Spike IgG at BL and after prime and boost vaccination ( $<35,2 \mathrm{BAU} / \mathrm{ml}$ : negative, $\geq 35,2$ $\mathrm{BAU} / \mathrm{ml}$ : positive). (E) Antibody neutralization activity is depicted as PRNT50 at BL, dpp and dpb vaccination for the SARS-CoV-2 ancestral variant B.1 and the VOC B.1.1.7 and B.1.351 at 13-16 and 28-30 $\mathrm{dpb}$ when the highest neutralization titer was detected with the ancestral B.1 variant. Numbers indicate median value (F) Dot plots showing double tetramer positive B cells for S1 and RBD epitope. (G) \% of spike-specific B cells depicted at BL, dpp and dpb as well as in natural infection for S1 and RBD epitope. (H) PBMC from BL, dpp and dpb as well as samples after natural infection were stimulated in vitro for nine days with CpG and IL-2. Secreted anti-SARS-CoV-2 Spike IgG was detected in the supernatant by ELISA. (I) Donut plots representing co-expression of CD27/lgG (upper) and CD71/CD95 (lower) in concatenated analysis of S1-specific $B$ cells populations ( $\mathrm{N}$ indicated the number of individual files concatenated) after boost vaccination. BL: baseline, dpp: days post prime, dpb: days post boost; TFH follicular helper T cells; TH1 T helper cells 1; PRNT50: plaque-reduction neutralization titer 50; BAU: antibody units; RU: arbitrary units. Bar charts show the median with IQR. 

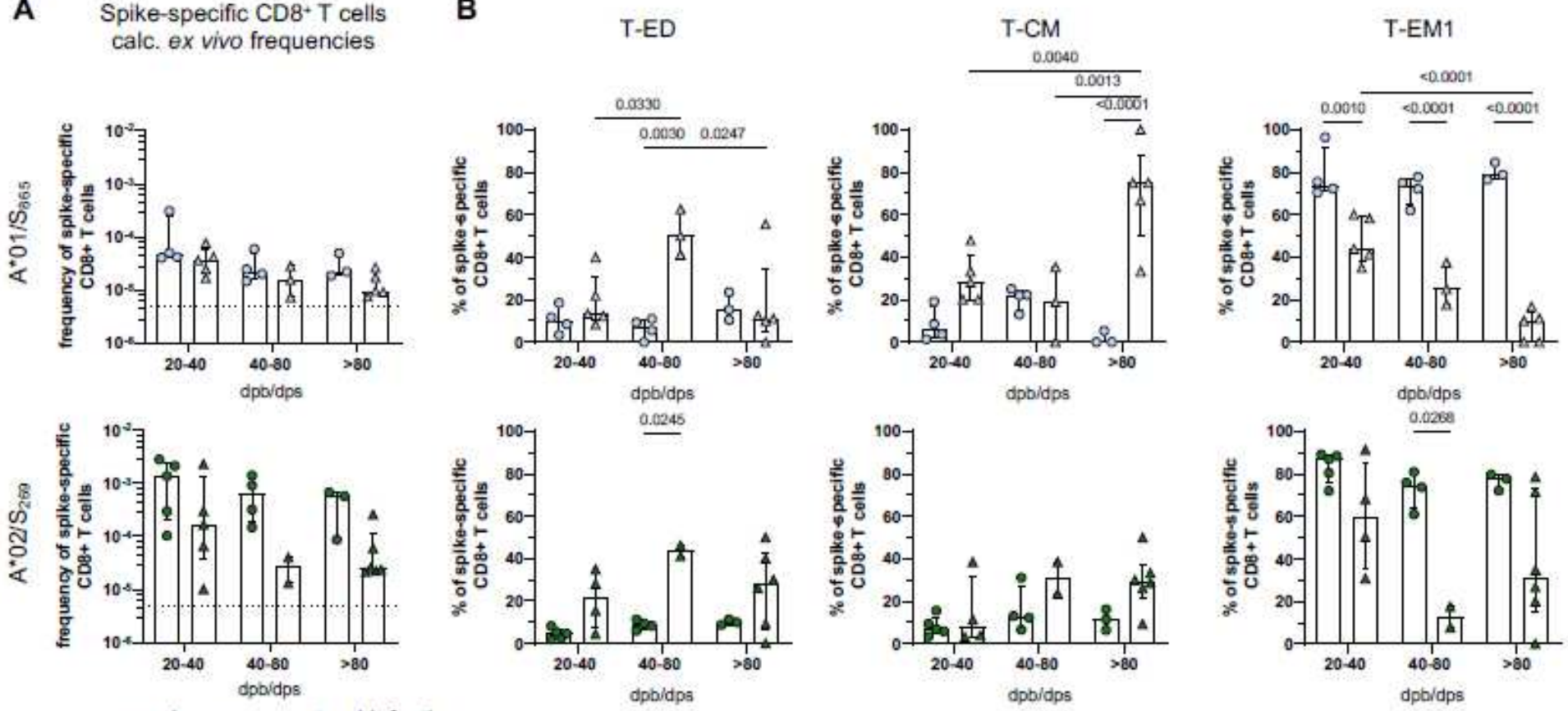
- $\mathrm{A}^{*} 01 / \mathrm{S}_{\mathrm{ses}}$
- $\mathrm{A}^{*} 02 / \mathrm{S}_{269}$
$\triangle A^{*} 01 / S_{965}$
A $\mathrm{A}^{*} 02 / \mathrm{S}_{269}$

natural infection

C
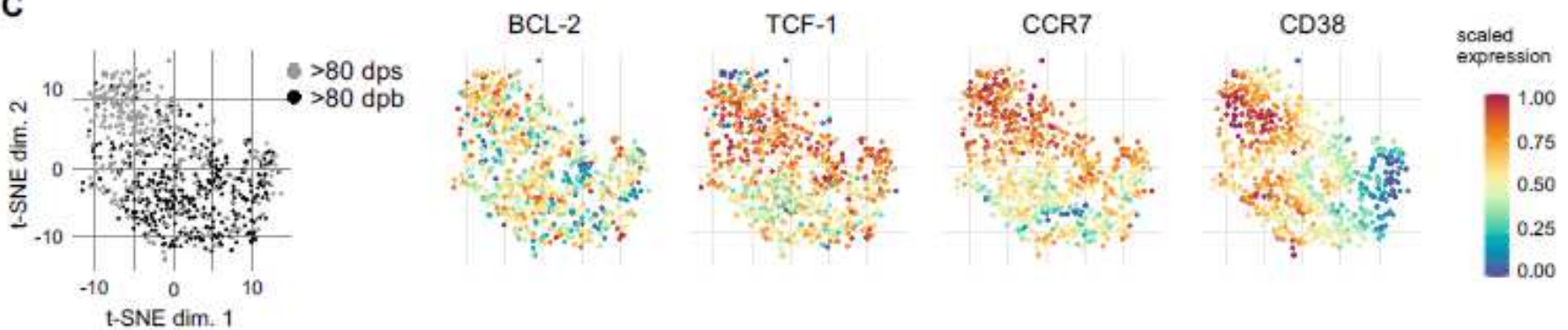

D
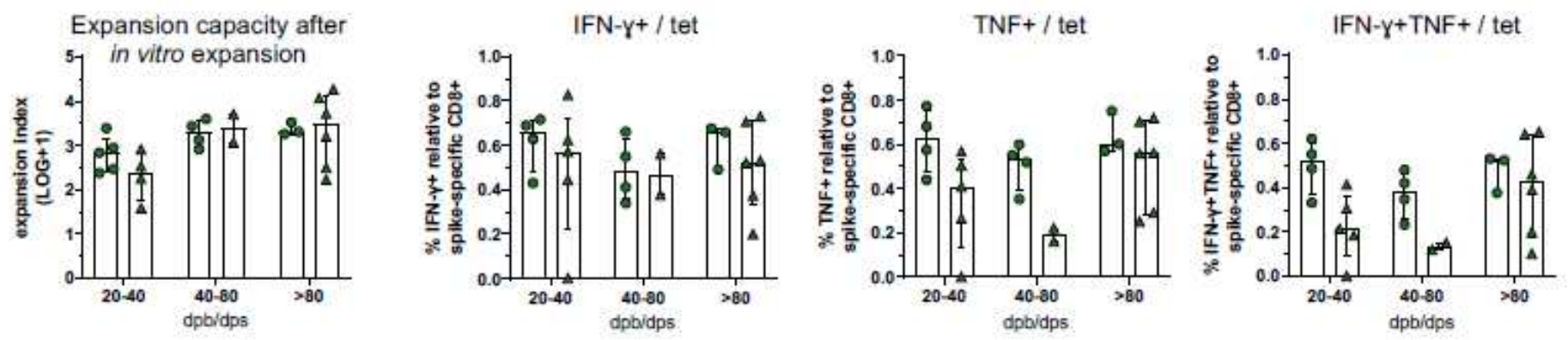

Figure 4

Differences of circulating spike-specific early memory CD8+ T cells after boost vaccination and natural infection (A) Calculated frequency of $A * 01 / S 865$ - and $A * 02 / S 269$-specific CD8+ $T$ cells after boost vaccination or natural SARS-CoV-2 infection ex vivo after $\mathrm{pMHCl}$ tetramer-based enrichment. (B) Distribution of CD8+ T cell memory subsets, TED, TCM and TEM1, for CD8+ T cells targeting A*01/S865and $A^{\star} 02 / S 269$-epitopes. (C) t-SNE representation of flow cytometry data, depicting spike-specific CD8+ T cells more than $80 \mathrm{dpb}$ and dps of natural infection (grey: natural infection, black: vaccines). BCL-2, TCF1, CCR7 and CD38 expression levels are plotted on the t-SNE (color-code: blue, low expression; red, high 
expression). (D) Frequency of the respective spike-specific CD8+ T cells after in vitro expansion. \% of IFN$\mathrm{Y}$ - and TNF-producing as well as IFN- $\mathrm{Y}$ and TNF co-producing CD8+ T cells in relation to the frequency of $A * 02 / S 269$-specific CD8+ T cells after in vitro expansion. Bar charts show the median with IQR. $A * 01 / S 865 n=5$ vaccinees longitudinally and $n=10$ naturally infected patients cross-

sectional/longitudinally; $A^{\star} 02 / S 269 n=5$ vaccinees longitudinally and $n=12$ naturally infected patients cross-sectional. 2way ANOVA including Tukey's multiple comparisons test were performed. dpb: days post boost; dps: days post symptoms; TED: early differentiated, TCM: central memory T cells and TEM1: effector memory $T$ cells 1 ; t-SNE: t-distributed stochastic neighbor embedding. 